\title{
Microscopic models of energy dissipation by internal degrees of freedom in particle collisions
}

\author{
Dissertation \\ zur Erlangung des Doktorgrades \\ der Mathematisch-Naturwissenschaftlichen Fakultäten \\ der Georg-August-Universität zu Göttingen
}

\author{
vorgelegt von \\ Timo Aspelmeier \\ aus Gütersloh
}

Göttingen, den 13. März 2000 
D7

Referentin: Prof. Dr. A. Zippelius

Korreferent: Prof. Dr. R. Kree

Tag der mündlichen Prüfung: 26. April 2000 


\section{Contents}

1. Introduction 3

I.1. Granular matter . . . . . . . . . . . . . . . . . . . . 3

1.2. Collision models . . . . . . . . . . . . . . . . . . . . . 6

2. One-dimensional particles 1: The two particle system 8

2.1. Review of the one-dimensional model . . . . . . . . . . . . . . . . . . 99 9

2.2. Characteristics of the transition probability . . . . . . . . . . . . . 14

2.3. Construction of an approximation for the transition probability . . . 19

2.4. Comparison with computer simulations . . . . . . . . . . 23

2.5. Summary . . . . . . . . . . . . . . . . . . . . . 24

3. One-dimensional particles 2: Many particle systems 26

3.1. The many particle model . . . . . . . . . . . . . . . . . . . . . 26

3.2. Computational methods . . . . . . . . . . . . . . . 27

3.3. Simulation results using the "exact" method . . . . . . . . . . . . . . 31

3.4. Comparison of many particle simulations using the numerical and analytical $p_{\beta}(\epsilon)$. . . . . . . . . . . . . . . . . 39

3.5. Application of $p_{\beta}(\epsilon)$ to many particle systems . . . . . . . . . . . 40

3.6. Summary . . . . . . . . . . . . . . . . . . . 46

4. From one to three dimensions 48

4.1. One-dimensional elastic model . . . . . . . . . . . . . . . . . . . . . . 48

4.2. Hard-core solution of the equations of motion . . . . . . . . . . . . . 49

4.3. Energy considerations . . . . . . . . . . . . . . . 51

$4.4 . \quad$ Two- and three-dimensional objects . . . . . . . . . . . . . . . 52

4.5. Differential equations of the free motion of an elastically vibrating body 57

4.6. Consequences of the finite set of modes . . . . . . . . . . . . 62

4.7. Summary . . . . . . . . . . . . . . . . . . . . . . . 63

5. Three-dimensional particles: Simulations 65

5.1. Vibrational modes of a sphere . . . . . . . . . . . . . . 66

5.2. Computational methods . . . . . . . . . . . . . . . 67

5.3. Simulation results . . . . . . . . . . . . . . . . . . . . . . . 69 
5.4. Summary . . . . . . . . . . . . . . . . . . 84

6. Conclusions and outlook 85

6.1 Conclusions . . . . . . . . . . . . . . . . 85

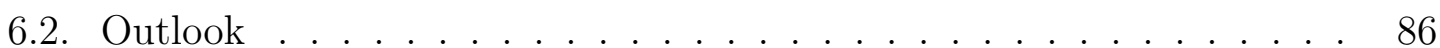

A. Details concerning the probability distribution $p_{\beta}(\epsilon) \quad \mathbf{8 8}$

A.1. Implications of the non-negativity of the probability density . . . . . 88

A.2. Evaluation of the low temperature limit . . . . . . . . . . . . . . 90

A.3. Evaluation of the high temperature limit . . . . . . . . . . . . . . 91

A.4. "Proof" for a uniform distribution at $\beta=0$. . . . . . . . . . . . . . 92

B. On the hard-core limit of the generalised one-dimensional collision equa$\begin{array}{ll}\text { tion } & 94\end{array}$

B.1. Solution of the equation of motion (2.12) in the hard-core limit . . . . 94

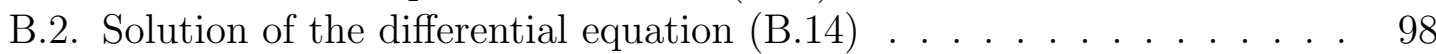

B.3. Properties of the function $F_{\alpha} \ldots \ldots \ldots$. . . . . . . . . . . . . . 99

\begin{tabular}{ll}
\hline C. Summary of Hertz' contact theory & 101
\end{tabular} 


\section{Introduction}

Collisions of macroscopic bodies like billiard balls, sand grains, or other familiar objects are frequently encountered by everybody. Despite their common occurence and apparent simplicity, real objects, in contrast to idealised bodies like e.g. hard spheres, can show a surprisingly complex collision behaviour. The outcome of a collision may depend on material, mass, shape, surface structure, elastic moduli, impact velocity, and other parameters [Bro.96]. One of the reasons for this complexity is the existence of static and sliding friction which acts between the touching surfaces; another reason is restitution, i.e. energy loss that occurs even if friction plays no role. In recent years, nanotribologists have tried to elucidate the microscopic origin of friction in its various guises [W(G.97]. The microscopic origin of restitution, however, has so far been mainly described phenomenologically only. This work therefore concentrates on one particular aspect of restitution, the production of vibrations by collisions, in order to derive macroscopic collision behaviour from a microscopic description.

Granular matter is one of the physically interesting (and at the same time technically important) fields where the non-ideal nature of particle collisions is crucial; it is responsible for many of the intriguing properties of such systems. A great deal of this work is therefore devoted to the application of the macroscopic collision laws derived from the microscopic description to granular many-particle systems. Consequently, the collision models proposed here are mainly discussed in the context of granular matter, even though they are of wider applicability.

In the following, an overview over the physics of granular matter will be given in order to set the stage for the more detailed discussions that are to follow in the main body of the text. A presentation of collision models used for modelling granular media and an explanation of the basic ideas of the models developed in this work conclude the introduction.

\subsection{Granular matter}

Granular materials have attracted the interest of many scientists over several centuries, including such famous physicists as Faraday, Coulomb, Reynolds, and many others. This is probably due to the fact that examples of granular media like sand, sugar, meal, soot, powder and the like can be found in everyday life, while at the 
same time they show an enormous variety of phenomena which more often than not elude a simple description. These phenomena include such diverse aspects as heap formation, giant force fluctuations, segregation, avalanches, dunes, the exotic "oscillons" and many more. For a general overview over granular materials and their behaviour, see e.g. [HHL.98, JN.92, JNB.96, dG.99]. To date, there exists no "unifying theory" which would describe all important aspects of granular materials in a general framework. There are even some who deny the very existence of such a theory [Kad99]. Nevertheless, it is worthwhile to investigate into the matter in order to illuminate at least a few corners of the whole picture. In recent years, much effort has been put into this; the renewed interest can be ascribed to the host of still unsolved questions and the increased number of theoretical, experimental, and computational methods and techniques that were not available to the early investigators.

Granular media are systems composed of a large number of possibly small but macroscopic particles. Therefore they obey purely classical behaviour. There are two main points that set granular materials apart from standard systems of statistical mechanics: a) Nonconservation of energy through dissipation and friction upon particle contact and b) irrelevance of the usual thermal energy scale $k_{\mathrm{B}} T$ [.JNB.96]. Thus, a granular system can be trapped in one of many meta-stable states where all particles are at rest due to static friction, such as e.g. a heap: It then behaves like a solid. When driven, however, the particles can move, showing fluid-like behaviour like e.g. in an avalanche. The fluid behaviour can roughly be divided into two regimes: When external driving is weak, particles are in permanent contact with each other, the dynamics being dominated by friction forces and steric constraints due to particle geometry. When the driving is strong, e.g. through gravity or vibrating plates, the particles are mostly flying freely and only interact through inelastic collisions. This is the so-called granular gas (or rapid flow or grain inertia) regime. When considering large assemblies of granular particles, this work will mainly focus on the latter; therefore a short overview over the physics of granular gases shall be given in the following.

\section{Granular gases}

"Real life" examples of granular gases can for instance be found in planetary rings [SHB.95, BWKH.96, SSK.97] or in the top layers of an avalanche. Much research has been done on these kinds of systems, experimentally, computationally, and theoretically. Some of the more recent experiments are e.g. to be found in [CLB ${ }^{+}$.93, WH.J95, [FFL.99]. The experiments are usually performed on a vibrating bottom plate in order to input the energy required to sustain a fluidized granular gas "phase" 7 at the top. Note also the interesting experiments described in [ $\left.\mathrm{FWE}^{+} 99\right]$ which were performed in low gravity, a situation which is closer to the one usually considered

\footnotetext{
${ }^{1}$ The quotation marks are to indicate that there is no precise definition of a granular gas which would unambigously distinguish it from slow flows.
} 
in theories and simulations. Numerous simulational studies of granular materials have been performed and discussed in the literature since many quantities of physical interest like density and velocity fields are very difficult to measure in experiments but easy to observe in simulations. Such simulations have been described in [GT793, MY92, MY.93, LCB ${ }^{+}$94, LHB.94, MY96, OBvNE.97, ML.98], to name but a few. The experiments and simulations are supplemented by theories: Gas kinetic theory was introduced into the field of granular matter by Savage, Jenkins, and others [S.I81, LS.JC8.3, .JS8.3, JR8.5a, JR8.56] and led to a hydrodynamic description in terms of coarse grained density, velocity, and energy fields. A similar result was also obtained by Haff [Haf83] using phenomenological arguments. Due to the dissipative grain interaction laws one has to employ non-equilibrium statistical mechanics methods in order to make any progress on the theoretical side. The most important methods to derive macroscopic theories from some basic assumptions about the constituents of the system originally come from gas kinetic theory, namely the Boltzmann equation and the Pseudo-Liouville operator, both extended to the case of inelastic collisions. There is an enormous amount of literature on these topics, only little of which will be cited here [CC60, Cer88, EDHvL69, vNEB98] since this will not be the primary interest of this work.

Many theoretical works consider the so-called homogeneous cooling state, i.e. they employ the assumption that the granular gas remains spatially homogeneous and has a velocity distribution whose shape is constant in time (it is usually, but not always [GS.5., Hut9.9], assumed to be a Gaussian2]) and which has only one scaling parameter, the granular temperature, which decays in time if no energy source is present. The term "granular temperature" is the literature standard for the mean kinetic energy of the particles, defined analogously to the temperature in an ordinary gas, although it is not a temperature in the thermodynamic sense. For rough or nonspherical particles, where rotations become important in collisions, it is necessary to introduce two temperatures, one for translational and one for rotational degrees of freedom [GS95, HZ.97, LHMZ98, HAZ.99]. The two temperatures in general decay differently: a clear manifestation of the nonequilibrium nature of such systems since in equilibrium systems one would find equipartition instead.

These theories have been formulated and extended in many ways and have led to an improved understanding of important features like clustering [G/9.3, McN.93, GBvNE.97], which is a consequence of the instability of the homogeneous cooling state against long wavelength density fluctuations and has been traced to noise reduction [BE.986]. Other aspects are still unsolved; e.g. a theory that remains valid in the clustering regime, away from the homogeneous cooling state, is still lacking, although attempts are being made in this direction [BE:98a, vNE.99, Hut.99].

\footnotetext{
${ }^{2}$ This assumption is also disproved by some experiments, e.g. [0099].
} 


\subsection{Collision models}

There are at least three levels on which one can look at a granular gas. From a macroscopic point of view, one is concerned with hydrodynamic properties like granular temperature, flow or density fields. The laws governing these quantities can be derived from a more mesoscopic level where individual grains are taken into account. In order to do so, one must introduce more or less phenomenological dissipative interaction rules for the particles. Systems of this type can be regarded as an open thermodynamic system with an energy sink [vNE.99]. Finally, one can look down to the level of the grains themselves and include the grains' microscopic dynamics in the description in order to account for the dissipative mechanisms. In this work, the latter point of view is taken. Starting from a microscopic description of individual grains, collision properties are derived, and many particle systems composed of such particles are studied.

Input to all the theories on the mesoscopic level are models for energy dissipation and friction. Very often, theories understandably use the simplest ones in order to keep calculations tractable; yet these models, the most prominent of which is the inelastic hard sphere (IHS) model, are often at best phenomenological and lack a sound theoretical basis (see [Rou98, Lud98, CR.98] for a discussion of several such models). The IHS model considers the granular particles to be spheres with constant coefficients of normal restitution $\epsilon$ and possibly tangential restitution $\beta$ ? This is certainly a rather crude approximation which is also invalidated by experiments [Gol60, FLCA.94, Dil93, HBL88, BSL ${ }^{+96}$ ]. As mentioned above, real granular particles show a very complex collision behaviour.

This discrepancy between the elaborate theories and their somewhat unsound foundations inspired the question of how and how much energy is lost in granular collisions under which circumstances. Several loss mechanisms can be identified: First, plastic deformation and fracture of the particles plays a role [And30, Tab48, Dil.93], i.e. collisions tend to permanently damage the particles such that energy will be irretrievably stored in lattice defects like dislocations. Second, the particles may be made of a viscoelastic material [Pao55, KK87, HSB95, BSHP96] such that energy is lost by internal friction in the material. Such an approach yields velocity dependent coefficients of restitution which can be fitted to agree with experimental results and which are of importance in e.g. the modeling of planetary rings [SHB.95, SSK.97]. Third, collisions of particles could excite elastic vibrations of the particles which take up energy. This effect is clearly visible in simple experiments (e.g. a hammer striking a bell) and has also been investigated for spheres hitting an elastic plate [Ram20, Zen41, Kol83]. Rayleigh [Ray06] estimated by an extension of Hertz' theory of contact [Her82] that the effect for spheres should be small. His estimate, however,

\footnotetext{
${ }^{3}$ The symbols $\epsilon$ and $\beta$ are going to appear again in the main text later on. Their exact definitions are therefore deferred until then.
} 
is only valid for identical spheres in a head-on collision. The ultimate aim of the present work is therefore to develop a method to go beyond Rayleigh's calculation and to check whether his estimate remains true under all conditions and to estimate the relative importance of the latter loss mechanism.

However, since the general case of colliding, elastically vibrating three-dimensional bodies is rather difficult to treat analytically (as will become clear in the text, see also [G79.9] for an approach in two dimensions), this work will start from a model of collisions of one-dimensional particles with internal, vibrational degrees of freedom. This model was introduced in [G/96, Gie.96] and has many intriguing properties worth studying in their own right. It led, for instance, to a stochastic description of the collision process with a fluctuating coefficient of restitution. It will be extended here in several respects: In Sec. 2 the exact solutions of the equations of motion for two such particles will be given and exploited in order to derive some exact properties of the probability density for the coefficient of restitution. Using these results, an analytical approximation will be constructed which can be used to perform accelerated simulations of many particle systems composed of these elastically vibrating particles. Simulations of such many particle systems will be presented in Ch. 3. Starting from the same equations of motion for the two particle system, but under more general conditions, a solution is presented in Ch. 1 which not only extends the result given before but is also easily generalisable to two and three dimensions. Hence by this calculation an approach to the problem of three-dimensional spheres (in principle even arbitrarily shaped bodies) becomes possible. The resulting expressions are still too complicated to permit a complete analytical solution but at least they allow for a relatively efficient numerical calculation of two body collisions, each body having vibrational degrees of freedom. Thus one is now in a position to test Rayleigh's estimate and to go beyond it by exploring the parameter space of radii, masses, poisson numbers, and other parameters. It is also possible to check the range of validity of Hertz' contact law [Her82] itself. Some first results of such simulations are presented in Ch. 罒. 


\section{One-dimensional particles 1: The two particle system}

In [GZ96, Gie.96], Giese and Zippelius introduced the model that forms the basis of the present work. Their model describes the end-to-end collision of two one-dimensional, elastically deformable particles (which will in the following occasionally be denoted as "rods"), interacting through a contact potential which forbids overlapping of the particles. Nevertheless, they can and in general will be elastically deformed. Through this mechanism translational energy is transferred to internal degrees of freedom during a collision. Consequently, collisions are in general inelastic (with respect to translational energy $E_{\mathrm{tr}}$ ) and can be characterised by a coefficient of restitution $\epsilon \neq 1$. The coefficient of restitution in one dimension is simply the ratio of the moduli of the relative particle velocities after and before the collision.

The statistical properties of binary collisions of one-dimensional, elastic rods were analysed in [G/96]. One of the main results concerns a separation of timescales: Equipartition among the vibrational modes is achieved much faster than the decay of the translational energy. This has led to modeling the internal degrees of freedom by a thermalised bath, characterised by a temperature $T_{\mathrm{B}}$. The binary collision process can then be reduced to a stochastic equation of motion for the relative velocity of the two particles. The duration of a collision as well as the relative velocity after collision are stochastic variables, depending on the state of the bath of each particle. The time evolution of $E_{\mathrm{tr}}$ upon successive collisions can be interpreted as a Markov process. During a collision, $E_{\mathrm{tr}}$ changes to a new value $E_{\mathrm{tr}}^{\prime}=E_{\mathrm{tr}} \epsilon^{2}$ with a transition probability $p_{T_{\mathrm{B}}}\left(E_{\mathrm{tr}} \rightarrow E_{\mathrm{tr}}^{\prime}\right)$.

In the following I will review the model and the associated concepts in Sec. 2.1 with regard to later extensions in Ch. 4 and will then proceed to show in Sec. 2.2 some exact analytic results for the probability distribution of the coefficient of restitution that can be derived from the model. Starting from these exact results I construct in Sec. 2.3 an analytic expression for a probability distribution which has the same properties in order to approximate the correct probability distribution. This nonstandard procedure was chosen because neither an exact nor even a perturbative calculation was possible due to the extreme nonlinearity of the hard-core potential. Finally, in Sec. 2.4, I compare the approximation with simulations in order to confirm that it shows all the desired features. 


\subsection{Review of the one-dimensional model}

The aim of this section is the equation of motion for the relative velocity of two colliding elastic rods, which was derived in [G/96, AG7.98b, AG/98a] and which will be reviewed here in some length for it also forms the basis of Sec. 4.1.

Two one-dimensional particles of lengths $l_{1}$ and $l_{2}$ with masses $m_{1}$ and $m_{2}$ move freely along a (circular) line until they collide. Their vibrational excitations are described by $N_{i}^{\text {mod }}$ normal modes $u_{i \nu}(s)\left(\nu=1, \ldots, N_{i}^{\text {mod }}\right.$ and $s \in\left[-l_{i} / 2, l_{i} / 2\right]$ denotes the position along the rod) of wavenumber $k_{i \nu}=\pi \nu / l_{i}$ and frequency $\omega_{i \nu}=c k_{i \nu}$ with amplitudes $q_{i \nu}$ and conjugate momenta $p_{i \nu}(i=1,2)$. The only important material parameter for this model is the sound velocity $c$. The centre-of-mass positions are denoted by $R_{1}$ and $R_{2}$, their distance is $R=R_{2}-R_{1}$, and their relative velocity is denoted by $\dot{R}$. Accordingly, $P=\mu \dot{R}$ is the relative momentum and $\mu=m_{1} m_{2} /\left(m_{1}+m_{2}\right)$ the effective mass. It is sometimes convenient to use dimensionless quantities when dealing with one-dimensional particles. This is done by dividing all lengths by $l=2 l_{1} l_{2} /\left(l_{1}+l_{2}\right)$, while times will be measured in units of $l / c$, i.e. $\tau=t c / l$ is the dimensionless time.

The Hamilton function of the system of two particles is then given by the sum of three contributions, the translational energy, the vibrational energy and the interaction potential:

$$
\begin{aligned}
\mathcal{H} & =\mathcal{H}_{\text {trans }}+\mathcal{H}_{\text {vib }}+V_{\alpha} \\
& =\frac{P^{2}}{2 \mu}+\sum_{i=1}^{2} \sum_{\nu=1}^{N_{i}^{\text {mod }}}\left\{\frac{p_{i \nu}{ }^{2}}{2 m_{i}}+m_{i} \omega_{i \nu}^{2} \frac{q_{i \nu}{ }^{2}}{2}\right\}+B e^{-\alpha r} .
\end{aligned}
$$

The interaction potential is modelled by a short-range repulsive potential $V_{\alpha}(r)=$ $B \exp (-\alpha r)$ (with positive but otherwise arbitrary $B$ ), which depends on the momentary end-to-end distance $r$ (to be defined below). Thereby translational and vibrational degrees of freedom are coupled. The parameter $\alpha$ controls the hardness of the potential. It will be sent to infinity later on in order to arrive at the hard-core limit.

\subsubsection{Derivation of the equations of motion}

The equations of motion are derived from the Hamilton function given in Eq. (2.1) in the usual way. Before they can be written down, one needs to know the distance $r$ of the endpoints of the rods, which is given by

$$
r=R-\left(l_{1}+l_{2}\right) / 2+\sum_{\nu=1}^{N_{2}^{\bmod }} q_{2 \nu} u_{2 \nu}\left(-l_{2} / 2\right)-\sum_{\nu=1}^{N_{1}^{\bmod }} q_{1 \nu} u_{1 \nu}\left(l_{1} / 2\right),
$$


where $R$ is the distance of the two centers of mass of the rods. The $\nu$-sums in Eq. (2.2) comprise the vibrational part of the end-to-end distance.

The equations of motion then read

$$
\begin{aligned}
\dot{P} & =\mu \ddot{R}=-V_{\alpha}^{\prime}(r) \\
\dot{p}_{i \nu} & =m_{i} \ddot{q}_{i \nu}=-m_{i} \omega_{i \nu}^{2} q_{i \nu}-(-1)^{i} V_{\alpha}^{\prime}(r) u_{i \nu}\left((-1)^{i+1} l_{i} / 2\right) .
\end{aligned}
$$

Putting Eq. (2.3) into Eq. (2.4) and letting $u_{i \nu}^{0}:=(-1)^{i} u_{i \nu}\left((-1)^{i+1} l_{i} / 2\right)$, one gets

$$
\ddot{q}_{i \nu}=-\omega_{i \nu}^{2} q_{i \nu}+u_{i \nu}^{0} \frac{\mu}{m_{i}} \ddot{R} .
$$

This linear differential equation describes a driven harmonic oscillator and can be solved with the Green function

$$
G_{i \nu}(t)=\Theta(t) \frac{\sin \left(\omega_{i \nu} t\right)}{\omega_{i \nu}}
$$

$(\Theta(\cdot)$ is the usual Heaviside step function) which yields, given the initial conditions $q_{i \nu}\left(T_{0}\right)$ and $p_{i \nu}\left(T_{0}\right)$ at some time $T_{0}$,

$$
\begin{aligned}
q_{i \nu}(t)=q_{i \nu}\left(T_{0}\right) \cos \left(\omega_{i \nu}\left(t-T_{0}\right)\right)+\frac{p_{i \nu}\left(T_{0}\right)}{m_{i} \omega_{i \nu}} \sin \left(\omega_{i \nu}\left(t-T_{0}\right)\right)+ \\
u_{i \nu}^{0} \frac{\mu}{m_{i}} \int_{T_{0}}^{t} G_{i \nu}\left(t-t^{\prime}\right) \ddot{R}\left(t^{\prime}\right) d t^{\prime}
\end{aligned}
$$

for $t \geq T_{0}$. The part stemming from the initial conditions will be abbreviated as $Q_{i \nu}(t)$. The initial time $T_{0}$ should be chosen such that the particles have not touched and are well seperated prior to $T_{0}$. It is to be considered as the starting time of the approach of the two particles.

The solution Eq. (2.7) is now put into Eq. (2.2), which is in turn inserted into Eq. (2.3) to give

$$
\ddot{R}=\frac{\alpha B}{\mu} e^{-\alpha(R+Q+(G * \ddot{R}))}
$$

where

$$
\begin{aligned}
& Q(t)=\sum_{i=1}^{2} \sum_{\nu=1}^{N_{i}^{\bmod }} u_{i \nu}^{0} Q_{i \nu}(t), \\
& G(t)=\sum_{i=1}^{2} \sum_{\nu=1}^{N_{i}^{\bmod }} \frac{\mu}{m_{i}}\left(u_{i \nu}^{0}\right)^{2} G_{i \nu}(t),
\end{aligned}
$$


and where $(\cdot * \cdot)$ denotes a convolution integral as in Eq. (2.7).

Eq. (2.7) can be brought into a more convenient form by writing

$$
R(t)=R\left(T_{0}\right)+\left(t-T_{0}\right) \dot{R}\left(T_{0}\right)+\int_{T_{0}}^{t}\left(t-t^{\prime}\right) \ddot{R}\left(t^{\prime}\right) d t^{\prime}
$$

and letting $\tilde{G}(t):=G(t)+t \theta(t)$, which then gives

$$
\ddot{R}(t)=\frac{\alpha B}{\mu} e^{-\alpha\left(R\left(T_{0}\right)+\left(t-T_{0}\right) \dot{R}\left(T_{0}\right)+(\tilde{G} * \ddot{R})(t)+Q(t)\right)}
$$

Note that the complicated-looking expression in the exponent is simply the end-toend distance of the two rods (multiplied by $-\alpha$ ).

From here one can proceed in two ways: The first is to explicitly calculate $\tilde{G}(t)$ in the limit $N_{i}^{\text {mod }} \rightarrow \infty$ for homogeneous rods, use it to solve Eq. (2.12) and afterwards carry out the hard core limit $\alpha \rightarrow \infty$. The second way is to keep $N_{i}^{\text {mod }}$ finite and solve Eq. (2.12) for $\alpha \rightarrow \infty$ I. Here, I will go the first way and defer the second one to Sec. 4.1. The reason is that the two approaches lead to different points of view of the collision process and have different areas of applicability. This will become clearer in Sec. 4.1.

By introducing the normalised velocity gain $w(t)=\left(\dot{R}\left(T_{0}\right)-\dot{R}(t)\right) / \dot{R}\left(T_{0}\right)$, one can write

$$
\begin{aligned}
(\tilde{G} * \ddot{R})(t) & =-\dot{R}\left(T_{0}\right)(\tilde{G} * \dot{w})(t) \\
& =-\dot{R}\left(T_{0}\right)\left(\tilde{G}^{\prime} * w\right)(t)
\end{aligned}
$$

by integration by parts. The summation of $\tilde{G}^{\prime}(t)$ can be performed exactly for the normal modes of homogeneous particles [Gie.96] and yields

$$
\tilde{G}^{\prime}(t)=\frac{l}{c}\left(\delta(t)+\sum_{i=1}^{2} \sum_{\nu=1}^{\infty} \delta\left(t-\frac{2 l_{i} \nu}{c}\right)\right),
$$

so that

$$
\left(\tilde{G}^{\prime} * w\right)(t)=\frac{l}{c}\left(w(t)+\sum_{i=1}^{2} \sum_{\nu=1}^{\infty} w\left(t-\frac{2 l_{i} \nu}{c}\right)\right) .
$$

Thus due to the term proportional to $\delta(t)$ in $\tilde{G}^{\prime}(t)$, the integral equation (2.12) becomes an ordinary differential equation containing memory terms (viz. the double

\footnotetext{
${ }^{1}$ One might conjecture that order of the limits $N_{i}^{\bmod } \rightarrow \infty$ and $\alpha \rightarrow \infty$ is not important. While this is likely to be true, however, it will be seen later that it has so far not been possible to prove.
} 
sum), which is, expressed in rescaled units of time $(\tau=t c / l)$ and with a suitable choice of the arbitrary but positive constant $B$,

$$
\frac{d}{d \tau} w(\tau)=\frac{1}{\kappa} \exp \left\{\kappa\left(\tau-\tau_{0}-w(\tau)-\sum_{i=1}^{2} \sum_{\nu=1}^{\infty} w\left(\tau-\nu \Gamma_{i}\right)+q(\tau)\right)\right\}
$$

where $\kappa=-\frac{\alpha l}{c} \dot{R}\left(T_{0}\right)$ and $\tau_{0}=c\left(R\left(T_{0}\right) / \dot{R}\left(T_{0}\right)+T_{0}\right) / l$. The initial conditions are contained in $q(\tau)=c Q(\tau l / c) /\left(l \dot{R}\left(T_{0}\right)\right)$. The $\Gamma_{i}$ are just constants and are determined by the length ratio $\gamma=l_{1} / l_{2}$ (assuming $l_{1}<l_{2}$ without loss of generality) of the rods according to $\Gamma_{1}=1+\gamma$ and $\Gamma_{2}=1+\frac{1}{\gamma}$.

Eq. (2.17) can be solved exactly by separation of variables. Subsequently, the hard core limit $(\alpha \rightarrow \infty$ or equivalently $\kappa \rightarrow \infty)$ can be performed by saddle point methods, yielding

$$
w(\tau)=\max \left(0, \max _{\tau^{\prime} \in[0, \tau]}\left\{\tau^{\prime}-\tau_{0}-\sum_{i=1}^{2} \sum_{\nu=1}^{\infty} w\left(\tau^{\prime}-\nu \Gamma_{i}\right)+q\left(\tau^{\prime}\right)\right\}\right) .
$$

This is the main result which will be used extensively in this chapter. The collision ends when the memory terms in Eq. (2.18) overcompensate the gain from the other terms $\tau^{\prime}-\tau_{0}+q\left(\tau^{\prime}\right)$, which are on average increasing. Fig. 2.1 shows a sample collision process in order to illustrate Eq. (2.18).

\subsubsection{Lasting contacts}

A first conclusion that can be drawn from Eq. (2.18) concerns the existence of lasting contacts between the endpoints of the two rods. The expression inside the parentheses on the rhs of Eq. (2.17) is again the end-to-end distance $r$ multiplied by $-\alpha$, since Eq. (2.17) is only a reformulation of Eq. (2.12),

$$
r(\tau)=\frac{l}{c} \dot{R}\left(T_{0}\right)\left(\tau-\tau_{0}-w(\tau)-\sum_{i=1}^{2} \sum_{\nu=1}^{\infty} w\left(\tau-\nu \Gamma_{i}\right)+q(\tau)\right) .
$$

This remains true in the hard-core limit $\alpha \rightarrow \infty$.

Whenever the expression inside the curly braces in Eq. (2.18) is monotonically increasing in $\tau^{\prime}$ in the vicinity of $\tau^{\prime}=\tau$ and simultaneously takes on its maximum at the right boundary of the interval $[0, \tau], w(\tau)$ simply follows this function. In other words,

$$
w(\tau)=\tau-\tau_{0}-\sum_{i=1}^{2} \sum_{\nu=1}^{\infty} w\left(\tau-\nu \Gamma_{i}\right)+q(\tau)
$$

in each time interval during which the rhs is monotonically increasing and larger than all previous values of $w(\tau)$. Comparison with Eq. (2.19) then shows that under 
these circumstances the end-to-end distance $r$ is zero. This corresponds to a lasting contact between the endpoints. Since this is the only situation in which $w(\tau)$ can actually increase, it follows that lasting contacts are the generic behaviour for collisions described by Eq. (2.18)2. A complete collision process will therefore be composed of periods in time where the endpoints touch, alternating with periods where they are separated.

\subsubsection{Stochastic description}

It was shown in [GZ96] that equipartition among the vibrational modes is established fast as compared to the decay of the translational energy. Based on this observation the internal degrees of freedom were modelled by a thermalised bath, characterised by a temperature $T_{\mathrm{B}}^{(i)}=E_{\mathrm{vib}}^{(i)} / N_{i}^{\text {mod }}$, where the vibrational energy of a $\operatorname{rod} i, E_{\mathrm{vib}}^{(i)}$, is given by the sum of the energies of the individual modes. While the number of modes, $N_{i}^{\text {mod }}$, was assumed to be infinite for the derivation of Eq. (2.18), it is reintroduced here for reasons that will become clear below. Under these assumptions the function $q(\tau)$ becomes a stochastic process since the initial conditions are no longer known precisely. It was shown in [G/96, Gie96] that $q(\tau)$ is the sum of two periodic Brownian bridge processes [KP92, p. 42] with periods $\Gamma_{1}$ and $\Gamma_{2}$ and is thus a Gaussian random process with zero mean. Its covariance is

$$
\begin{aligned}
C_{q}(\tau) & =\left\langle q\left(\tau^{\prime}\right) q\left(\tau^{\prime}+\tau\right)\right\rangle \\
& =\sum_{i=1}^{2}\left\{\frac{1}{2 \Gamma_{i}}\left(\tau-\frac{\Gamma_{i}}{2}-\Gamma_{i} \sum_{\nu=1}^{\infty} \theta\left(\tau-\nu \Gamma_{i}\right)\right)^{2}-\frac{\Gamma_{i}}{24}\right\} \frac{T_{\mathrm{B}}^{(i)}}{2 E_{\mathrm{tr}}} .
\end{aligned}
$$

Here $E_{\mathrm{tr}}=(P(0))^{2} / 2 \mu$ is the translational energy of the colliding rods before the collision.

Since Eq. (2.18) has now become a stochastic equation, all observable quantities become stochastic as well, e.g. the duration of the collision as well as the final velocity or the coefficient of restitution. The latter two are related by

$$
\epsilon=\lim _{\tau \rightarrow \infty} w(\tau)-1
$$

If the two particles are installed on a circular line such that they perform an infinite series of collisions with each other as they travel back and forth on the circle, this can be interpreted as a Markov process in discrete time which accounts for transitions of the translational energy upon successive collisions. During a collision, $E_{\mathrm{tr}}$ changes to a new value $E_{\mathrm{tr}}^{\prime}=E_{\mathrm{tr}} \epsilon^{2}$. The probability density $p_{T_{\mathrm{B}}}\left(E_{\mathrm{tr}} \rightarrow E_{\mathrm{tr}}^{\prime}\right)$ for

${ }^{2}$ If $q(\tau)$ is a gaussian random process, as will be the case below, this may not be strictly true since in that case, $q(\tau)$ may be nowhere monotonically increasing. But since this is only a mathematical idealisation, this objection can be ignored for the present argument. 
this transition is connected to the probability density for the coefficient of restitution $p_{\beta}(\epsilon)$ according to

$$
p_{T_{\mathrm{B}}}\left(E_{\mathrm{tr}} \rightarrow E_{\mathrm{tr}}^{\prime}\right)=\left.\frac{1}{2 \epsilon E_{\mathrm{tr}}} p_{\beta}(\epsilon)\right|_{\epsilon=\sqrt{\frac{E_{\mathrm{tr}}^{\prime}}{E_{\mathrm{tr}}}}} .
$$

Here $\beta=E_{\mathrm{tr}} / T_{\mathrm{B}}$. For notational simplicity, no distinction is made between the temperatures of the individual rods, assuming they are equal.

Changes in the bath temperature are not independent, but determined by energy conservation:

$$
T_{\mathrm{B}}^{\prime}=T_{\mathrm{B}}+\frac{1-\epsilon^{2}}{\sum_{i} N_{i}^{\bmod }} E_{\mathrm{tr}}
$$

Note that one drawback of Eqs. (2.17) and (2.18) is that in their derivation it was necessary to use an infinite number of modes. Later, one had to return to a finite number of modes, otherwise the bath temperatures of the rods would never change (cf. Eq. (2.24)). This problem will be remedied in Ch. 4 .

The stationary state of the Markov process is known: after cooling (i.e. starting from an initial state that has $T_{\mathrm{B}}=0$ ), the system of two particles, each equipped with an internal bath, evolves into a stationary state with a Boltzmann distribution for $E_{\mathrm{tr}}$ and with bath temperature $T_{\mathrm{B}}^{0}=\frac{E_{\mathrm{tot}}}{2 N_{\mathrm{mod}}+1 / 2}$ where $E_{\mathrm{tot}}$ is the total energy of the system.

\subsection{Characteristics of the transition probability}

In this section some exact properties of the transition probability introduced in the previous section are derived from Eq. (2.18). The approximation to this probability that is going to be built at the end of this chapter will be constructed in such a way that it obeys all of these properties.

\subsubsection{Detailed balance}

Since the underlying model is microscopically reversible, detailed balance holds for the Markov process: the transition probability for the process $E_{\mathrm{tr}} \rightarrow E_{\mathrm{tr}}^{\prime}=E_{\mathrm{tr}} \epsilon^{2}$ is related to the transition probability for the reverse process $E_{\mathrm{tr}}^{\prime} \rightarrow E_{\mathrm{tr}}=E_{\mathrm{tr}}^{\prime} / \epsilon^{2}$ via

$$
p_{T_{\mathrm{B}}^{0}}\left(E_{\mathrm{tr}} \rightarrow E_{\mathrm{tr}}^{\prime}\right) e^{-E_{\mathrm{tr}} / T_{\mathrm{B}}^{0}}=p_{T_{\mathrm{B}}^{0}}\left(E_{\mathrm{tr}}^{\prime} \rightarrow E_{\mathrm{tr}}\right) e^{-E_{\mathrm{tr}}^{\prime} / T_{\mathrm{B}}^{0}}
$$

Here fluctuations of the temperatures of the baths have been neglected which seems justified because changes in the bath temperature are $\mathcal{O}\left(1 / N_{i}^{\text {mod }}\right.$ ) (see Eq. (2.24)). 
Detailed balance, Eq. (2.25), implies the following relation for $p_{\beta}(\epsilon)$ :

$$
p_{\beta}(\epsilon) e^{-\beta}=p_{\epsilon^{2} \beta}\left(\frac{1}{\epsilon}\right) e^{-\epsilon^{2} \beta}
$$

Since detailed balance is a property of the equilibrium state of the two particle system, this is strictly valid only if the temperatures of both rods are equal. Later, for lack of a better scheme, it will also be used for rods with different bath temperatures.

\subsubsection{High and low temperature limits}

When the temperatures of the baths of oscillators are zero, i.e. $q(\tau)=0$ for all $\tau$, the collision is deterministic. For this case, Eq. (2.18) can be solved exactly "by inspection": As long as the expression inside the curly braces in Eq. (2.18) is monotonically increasing, which it is for $\tau^{\prime}<\tau_{0}+\Gamma_{1}$ (recall that $\Gamma_{1}<\Gamma_{2}$ ), the inner max-function is redundant such that $w(\tau)=\left(\tau-\tau_{0}\right) \Theta\left(\tau-\tau_{0}\right)$ for $\tau<\tau_{0}+\Gamma_{1}$. For larger $\tau$, the memory terms compensate all the gain from the term $\tau-\tau_{0}$, thus the final value of $w(\tau)$ is $\Gamma_{1}$. Hence it follows that the coefficient of restitution is $\epsilon=\Gamma_{1}-1=\gamma$, the length ratio of the rods. This is well known and has been shown e.g. in [Aue.94, G/96]. Apparently, the earliest derivation of this result seems to be due to Saint-Venant as early as 1867, according to the reference on p. 283 in Rayleigh's article $[\text { Ray06 }]^{3}$. Thus in the limit of small temperatures, $p_{\beta}(\epsilon)$ should approach a $\delta$-Function centered around $\gamma$.

At large temperatures, on the other hand, $p_{\beta}(\epsilon)$ is a uniform distribution, i.e. $p_{\beta=0}(\epsilon)=$ const. (within a certain range and zero outside of it). This is proved in App. A.4.

\subsubsection{Maximum collision time}

If the length ratio $\gamma$ is a rational number, it is possible to extract some more information about $p_{\beta}(\epsilon)$ from Eq. (2.18). First one needs to know the maximum duration of a collision before an upper and lower bound for $\epsilon$ can be deduced.

The stochastic process $q(\tau)$ consists of two periodic Brownian bridge processes with periods $\Gamma_{1}$ and $\Gamma_{2}$ respectively. If $\gamma$ is rational, say $\gamma=p / s$ (where the integers $p$ and $s$ are relatively prime), then $\Gamma_{1} / \Gamma_{2}=p / s$ is rational and thus $q(\tau)$ is periodic, the period being given by $p+s$. Let $\tau^{*}$ be the time where $\tau-\tau_{0}+q(\tau)$ first equals 0 and is greater than 0 in an (arbitrarily small) interval to the right of $\tau^{*}$ (see Fig. 2.1). In other words, $\tau^{*}$ is the time when the particles first touch and the collision begins. The periodicity of $q(\tau)$ now leads to a maximum collision time because whatever

\footnotetext{
${ }^{3}$ A slightly more involved calculation than the one mentioned here shows that amazingly $w(\tau)$ assumes the same final value $\Gamma_{1}$ even if only the longer rod is nonvibrating while the shorter one vibrates with arbitrary strength.
} 


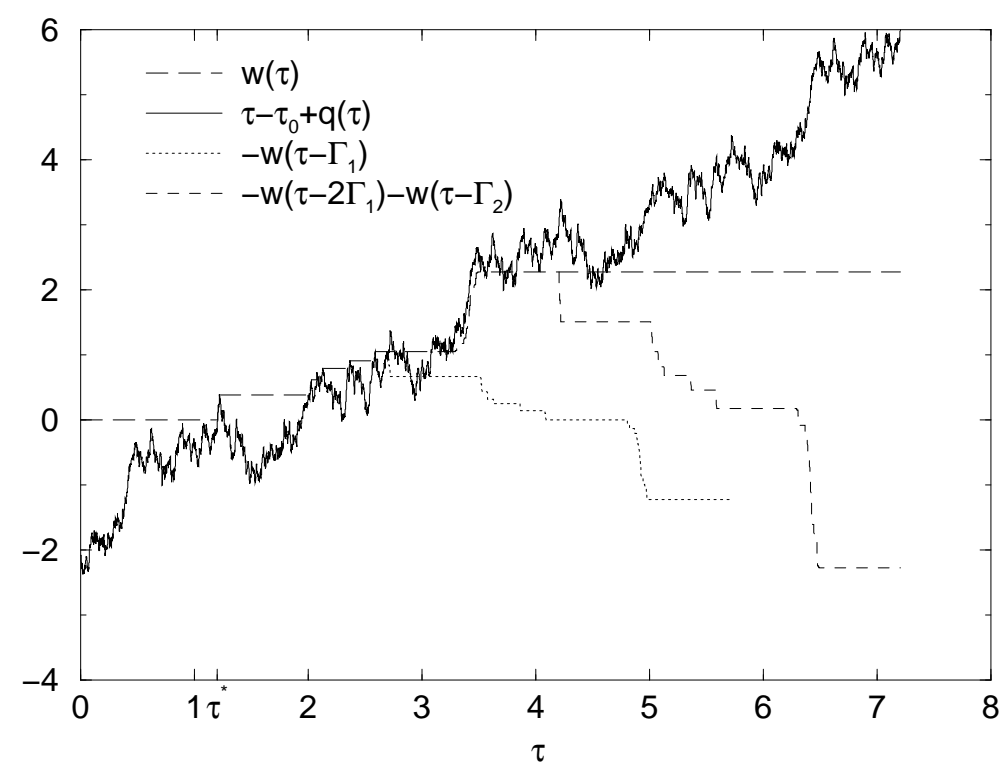

Figure 2.1.: Sample collision process for $\gamma=1 / 2$, i.e. $\Gamma_{1}=3 / 2$ and $\Gamma_{2}=3$. The stochastic process $q(\tau)$ is periodic with period 3. The memory terms (the dotted and short dashed curves) are attached to the curve for $w(\tau)$ for illustration. The time $\tau^{*}$ marks the beginning of the collision.

$w(\tau)$ is for a particular realisation of $q(\tau)$, the memory terms which accumulate overcompensate the gain from the term $\tau^{\prime}-\tau_{0}+q\left(\tau^{\prime}\right)$ after time $\tau^{*}+p+s$ (see Eq. (2.18) and Fig. 2.1 for illustration).

This can be seen more clearly by the following argument. By evaluating Eq. (2.18) at $\tau=\tau^{*}+p+s$ it is clear that

$$
\begin{aligned}
\tilde{w} & :=w\left(\tau^{*}+p+s\right) \\
& =\max _{\tau^{\prime} \in\left[0, \tau^{*}+p+s\right]}\left\{\tau^{\prime}-\tau_{0}-\sum_{i=1}^{2} \sum_{\nu=1}^{\infty} w\left(\tau^{\prime}-\nu \Gamma_{i}\right)+q\left(\tau^{\prime}\right)\right\} \\
& \geq \tau^{*}+p+s-\tau_{0}-\sum_{i=1}^{2} \sum_{\nu=1}^{\infty} w\left(\tau^{*}+p+s-\nu \Gamma_{i}\right)+q\left(\tau^{*}+p+s\right) \\
& =p+s-\sum_{i=1}^{2} \sum_{\nu=1}^{\infty} w\left(\tau^{*}+p+s-\nu \Gamma_{i}\right) .
\end{aligned}
$$

The last step follows from the definition of $\tau^{*}$ and the periodicity of $q(\tau)$. When the expression inside the curly braces in Eq. (2.18) is rewritten in the following way by splitting the double sum in three parts, one for $i=1$ and $\nu \leq s$, the second one for $i=2$ and $\nu \leq p$, and the third one containing the rest (keeping in mind that 
$s \Gamma_{1}=p \Gamma_{2}=p+s$ and renumbering appropriately),

$$
\begin{aligned}
& \tau^{\prime}-\tau_{0}+q\left(\tau^{\prime}\right)-\sum_{i=1}^{2} \sum_{\nu=1}^{\infty} w\left(\tau^{\prime}-\nu \Gamma_{i}\right)= \\
& \left(\tau^{\prime}-(p+s)\right)-\tau_{0}+q\left(\tau^{\prime}-(p+s)\right)-\sum_{i=1}^{2} \sum_{\nu=1}^{\infty} w\left(\tau^{\prime}-(p+s)-\nu \Gamma_{i}\right) \\
& \quad-\sum_{\nu=1}^{s} w\left(\tau^{\prime}-\nu \Gamma_{1}\right)-\sum_{\nu=1}^{p} w\left(\tau^{\prime}-\nu \Gamma_{2}\right)+p+s,
\end{aligned}
$$

the last four terms can be estimated by use of Ineq. (2.27): $p+s-\sum_{\nu=1}^{s} w\left(\tau^{\prime}-\nu \Gamma_{1}\right)-$ $\sum_{\nu=1}^{p} w\left(\tau^{\prime}-\nu \Gamma_{2}\right) \leq \tilde{w}$, first only for $\tau^{\prime}=\tau^{*}+p+s$ but since $w(\tau)$ is a monotonically increasing function, this also applies for all $\tau^{\prime}>\tau^{*}+p+s$. From the two sums the last terms could even be dropped because they are zero for $\tau^{\prime}=\tau^{*}+p+s$. However, only one of these last terms will be dropped, resulting in

$$
p+s-\sum_{\nu=1}^{s-1} w\left(\tau^{\prime}-\nu \Gamma_{1}\right)-\sum_{\nu=1}^{p} w\left(\tau^{\prime}-\nu \Gamma_{2}\right) \leq \tilde{w} \quad \text { for } \tau^{\prime} \geq \tau^{*}+p+s .
$$

Thus the rhs of Eq. (2.28) can be estimated as follows (again for $\tau^{\prime} \geq \tau^{*}+p+s$ ):

$$
\begin{gathered}
\tau^{\prime}-(p+s)-\tau_{0}+q\left(\tau^{\prime}-(p+s)\right)-\sum_{i=1}^{2} \sum_{\nu=1}^{\infty} w\left(\tau^{\prime}-(p+s)-\nu \Gamma_{i}\right) \\
-\sum_{\nu=1}^{s} w\left(\tau^{\prime}-\nu \Gamma_{1}\right)-\sum_{\nu=1}^{p} w\left(\tau^{\prime}-\nu \Gamma_{2}\right)+p+s \leq \\
\tau^{\prime}-(p+s)-\tau_{0}+q\left(\tau^{\prime}-(p+s)\right)-\sum_{i=1}^{2} \sum_{\nu=1}^{\infty} w\left(\tau^{\prime}-(p+s)-\nu \Gamma_{i}\right) \\
-w\left(\tau^{\prime}-(p+s)\right)+\tilde{w} \leq \tilde{w} \cdot(2.30)
\end{gathered}
$$

The last inequality again follows from Eq. (2.18), evaluated at $\tau=\tau^{\prime}-(p+s)$ : $w\left(\tau^{\prime}-(p+s)\right)$ (which is the one term that was kept) is greater than or equal to $\tau^{\prime}-(p+s)-\tau_{0}+q\left(\tau^{\prime}-(p+s)\right)-\sum_{i=1}^{2} \sum_{\nu=1}^{\infty} w\left(\tau^{\prime}-(p+s)-\nu \Gamma_{i}\right)$. Since the lhs of Ineq. (2.30) was just the expression in curly braces from Eq. (2.18) and $\tilde{w}=$ $w\left(\tau^{*}+p+s\right)$, this means that no further contributions to $w(\tau)$ come from times $\tau>\tau^{*}+p+s$ and it therefore follows that $p+s$ is the maximum collision time.

\subsubsection{Upper and lower bound for $\epsilon$}

Since the maximum collision time is $p+s$, we have $\epsilon=w\left(\tau^{*}+p+s\right)-1$ (cf. Eq. (2.22)). Bounds on $\epsilon$ can now be derived from Eq. (2.18), evaluated at $\tau=\tau^{*}+p+s$, by neglecting or over-estimating the memory terms. 
Consider first Eq. (2.18) at $\tau=\tau^{*}+p+s$ with all memory terms neglected which yields the following inequality:

$$
w\left(\tau^{*}+p+s\right) \leq \max \left(0, \max _{\tau^{\prime} \in\left[0, \tau^{*}+p+s\right]}\left\{\tau^{\prime}-\tau_{0}+q\left(\tau^{\prime}\right)\right\}\right) .
$$

Since $\tau^{*}$ is the beginning of the collision, it is clear that $\tau^{\prime}-\tau_{0}+q\left(\tau^{\prime}\right) \leq 0$ for all $\tau^{\prime} \leq \tau^{*}$. Thus it is also clear that $\tau^{\prime}-\tau_{0}+q\left(\tau^{\prime}\right) \leq p+s$ for all $\tau^{\prime} \leq \tau^{*}+p+s$ (simply by replacing $\tau^{\prime}$ by $\tau^{\prime}-p-s$ and using the periodicity of $\left.q(\tau)\right)$. At time $\tau^{\prime}=\tau^{*}+p+s$, this inequality even becomes an equality. Therefore, the rhs of Eq. (2.31) is equal to $p+s$, so one gets

$$
\epsilon+1=w\left(\tau^{*}+p+s\right) \leq p+s
$$

Next, the at most $p+s-2$ non-zero memory terms in Eq. (2.18) at time $\tau^{*}+p+s$ are over-estimated by the maximum possible value, namely $w\left(\tau^{*}+p+s\right)=\epsilon+1$ :

$$
w\left(\tau^{*}+p+s\right) \geq \max \left(0, \max _{\tau^{\prime} \in\left[0, \tau^{*}+p+s\right]}\left\{\tau^{\prime}-\tau_{0}-(p+s-2)(\epsilon+1)+q\left(\tau^{\prime}\right)\right\}\right) .
$$

By the same argument as above, the rhs of Eq. (2.33) is equal to $p+s-(p+s-2)(\epsilon+1)$. Thus one has

$$
\begin{aligned}
\epsilon+1=w\left(\tau^{*}+p+s\right) & \geq p+s-(p+s-2)(\epsilon+1) \\
\Leftrightarrow \quad \epsilon & \geq \frac{1}{p+s-1}
\end{aligned}
$$

It is easy to see that the upper and lower bounds on $\epsilon$ are also compatible with the detailed balance condition, Eq. (2.26), as it should be.

Because it will be needed later on, the parameter $\xi$ is introduced here for convenience:

$$
\xi=\frac{1}{p+s-1}
$$

The bounds calculated in this section are optimal bounds. This follows from the fact that $p_{\beta=0}(\epsilon)$ is a uniform distribution between $\xi$ and $1 / \xi$ which is proved in App. A.4.

\subsubsection{The special case $\gamma=1$}

When the length ratio of the rods, $\gamma$, is equal to 1 , an exact solution for $p_{\beta}(\epsilon)$ can be deduced from the results of the preceding sections. If $\gamma=1$, the periods of the two 
processes that constitute $q(\tau)$ are $p=s=1$, which results in the following upper and lower bounds for $\epsilon$ :

$$
\begin{aligned}
& \text { Upper limit: } \epsilon \leq p+s-1=1 \\
& \text { Lower limit: } \epsilon \geq \frac{1}{p+s-1}=1 .
\end{aligned}
$$

In other words, if $\gamma=1$, the probability density is given by

$$
p_{\beta}(\epsilon)=\delta(\epsilon-1) \quad \text { for all } \beta
$$

exactly. Note that this result implies the interesting and rather counter-intuitive fact that no matter how the two rods vibrate prior to the collision, the outcome is always $\epsilon=1$.

\subsection{Construction of an approximation for the transition probability}

As a short summary, the following properties of the probability density $p_{\beta}(\epsilon)$ have been established so far:

$$
\begin{array}{rc}
\text { Normalisation: } & \int_{0}^{\infty} p_{\beta}(\epsilon) d \epsilon=1 \\
\text { Positivity: } & p_{\beta}(\epsilon) \geq 0 \\
\text { Low temperature limit: } & \lim _{\beta \rightarrow \infty} p_{\beta}(\epsilon)=\delta(\epsilon-\gamma) \\
\text { High temperature limit: } & \lim _{\beta \rightarrow 0} p_{\beta}(\epsilon)=\text { const. within bounds } \\
\text { Upper and lower bounds: } & p_{\beta}(\epsilon)=0 \text { for } \epsilon<\xi \text { and } \epsilon>\frac{1}{\xi} \\
\text { Detailed balance: } & p_{\beta}(\epsilon) e^{-\beta}=p_{\epsilon^{2} \beta}\left(\frac{1}{\epsilon}\right) e^{-\epsilon^{2} \beta}
\end{array}
$$

It is desirable to know the functional form of $p_{\beta}(\epsilon)$ explicitly because that would greatly speed up simulations of a many particle system. Without it, simulations rely on a numerical solution of Eq. (2.18) with a randomly generated process $q(\tau)$ at every collision event. This is a very time consuming method which doesn't allow for a many particle system to be simulated over very long times. Therefore I now want to construct an analytical approximation for $p_{\beta}(\epsilon)$ which fulfills all of the above conditions Eq. (2.39) - (2.44). I will restrict myself to rational $\gamma$ since Eq. (2.43), which will prove to be quite useful in the following, applies only for this case.

\subsubsection{General solution of the detailed balance equation}

First, the detailed balance equation (2.44) is rewritten in terms of the probability density $p(x, y)$ for the variable $x=\beta \epsilon^{2} / 2$ where the parameter $y$ is given by $y=\beta / 2$. 
Then Eqs. (2.39) and (2.44) read:

$$
\begin{aligned}
\int_{0}^{\infty} p(x, y) d x & =1 \\
p(x, y) e^{-2 y} & =p(y, x) e^{-2 x}
\end{aligned}
$$

It is easy to check that the general solution of these two equations together is given by

$$
p(x, y)=e^{2 y} \frac{\partial^{2}}{\partial x \partial y} c(x, y)
$$

where $c(x, y)$ is an arbitrary real function which is symmetric with respect to interchange of its arguments and with the additional property that

$$
\begin{aligned}
c(0, y) & =\frac{1}{2} e^{-2 y} \quad \text { and } \\
\lim _{x \rightarrow \infty} c(x, y) & =0 \text { for all } y .
\end{aligned}
$$

So far, Eq. (2.47) does not guarantee that $p(x, y)$ is non-negative everywhere nor does it give any clue as to how to choose $c(x, y)$ such that the limits given in Eqs. (2.41) and (2.42) are fulfilled. In order to get an idea how to accomplish this, let's take a look at the known solution of $p_{\beta}(\epsilon)$ for the case $\gamma=1$, see Eq. (2.38). Expressed in terms of $x$ and $y$, this gives

$$
p(x, y)=\delta(x-y)
$$

It can be checked that this function is generated using

$$
c(x, y)=\frac{1}{2} e^{-x-y-|x-y|}
$$

in Eq. (2.47).

This expression suggests a way to reformulate Eq. (2.47) for arbitrary $\gamma$. As will be proved in App. A.1, $c(x, y)$ may be written without loss of generality in the form

$$
c(x, y)=\frac{1}{2} e^{-x-y-f(x, y)}
$$

where $f(x, y)$ is a real, symmetric function of its arguments with additional constraints $f(0, y)=y$ and $\lim _{x \rightarrow \infty}(x+f(x, y))=\infty$ in order to satisfy Eq. (2.48). For $\gamma \lesssim 1$ this new function $f(x, y)$ is expected to be approximately equal to $|x-y|$ but it is also expected to be differentiable for all $\gamma<1$. The ansatz of Eq. (2.51) gives

$$
\begin{aligned}
p(x, y) & =\frac{1}{2} e^{2 y} \frac{\partial^{2}}{\partial x \partial y} e^{-x-y-f(x, y)} \\
& =\frac{1}{2} e^{y-x-f(x, y)}\left(\left(1+\frac{\partial f}{\partial x}\right)\left(1+\frac{\partial f}{\partial y}\right)-\frac{\partial^{2} f}{\partial x \partial y}\right) .
\end{aligned}
$$


Now a function $f(x, y)$ has to be found which generates a $p(x, y)$ with all requirements specified by Eqs. (2.39) - (2.44).

As shown in App. A.1, the non-negativity of $p(x, y)$ can be guaranteed by choosing $f(x, y)$ such that $\partial^{2} f /(\partial x \partial y) \leq 0$ for all $x, y$. It is also shown in App. A.1 that this condition implies $|\partial f / \partial x| \leq 1$ for all $x, y$. Although this may not be the most general choice of $f(x, y)$, simulations suggest that the "real" $f(x, y)$ behaves in just this way.

The upper and lower limits for $\epsilon$, Eq. (2.43), require that

$$
f(x, y)=|x-y| \quad \text { exactly for } x<\xi^{2} y \text { and } x>\xi^{-2} y \text {. }
$$

So all that remains is to construct $f(x, y)$ in the range $\xi^{2}<x / y<\xi^{-2}$ in such a way that the high and low temperature limits are fulfilled.

\subsubsection{Low temperature limit}

First the average values of $x$ and $\sqrt{x}$ are computed from Eq. (2.52):

$$
\begin{aligned}
\langle x\rangle & =\frac{1}{2} e^{y} \int_{0}^{\infty}\left(1+\frac{\partial f}{\partial y}\right) e^{-x-f(x, y)} d x \\
\langle\sqrt{x}\rangle & =\frac{1}{4} e^{y} \int_{0}^{\infty}\left(1+\frac{\partial f}{\partial y}\right) e^{-x-f(x, y)} \frac{d x}{\sqrt{x}}
\end{aligned}
$$

These are related to the average values of $\epsilon$ and $\epsilon^{2}$ by

$$
\begin{aligned}
\langle\epsilon\rangle & =\frac{\langle\sqrt{x}\rangle}{\sqrt{y}} \\
\left\langle\epsilon^{2}\right\rangle & =\frac{\langle x\rangle}{y} .
\end{aligned}
$$

Thus for Eq. (2.41) to hold we must have $\langle\epsilon\rangle^{2}=\left\langle\epsilon^{2}\right\rangle=\gamma^{2}$ as $\beta \rightarrow \infty$. Expressed in terms of $x$ and $y$ this yields

$$
\begin{aligned}
\lim _{y \rightarrow \infty} \frac{\langle\sqrt{x}\rangle}{\sqrt{y}} & =\gamma \\
\lim _{y \rightarrow \infty} \frac{\langle x\rangle}{y} & =\gamma^{2} .
\end{aligned}
$$

It is shown in App. A.2 that this condition is satisfied if $f(z y, y)$ has the following property:

$$
\lim _{y \rightarrow \infty} y(1-z)-f(z y, y)= \begin{cases}0 & \text { if } 0 \leq z<\gamma^{2} \\ -\infty & \text { if } \gamma^{2}<z \leq 1\end{cases}
$$




\subsubsection{High temperature limit}

First, Eq. (2.52) is reformulated in terms of a new function $\tilde{f}(z, y)$ which is defined by

$$
y \tilde{f}(z, y)=f(z y, y)
$$

The symmetry condition implies $\tilde{f}(z, y)=z \tilde{f}(1 / z, z y)$. Eq. (2.53) requires that $\tilde{f}(z, y)=|1-z|$ for $z \leq \xi^{2}$ and $z \geq \xi^{-2}$. This then gives, after translation into an equation for the original variables $\epsilon$ and $\beta$,

$$
\begin{aligned}
p_{\beta}(\epsilon)= & \frac{\epsilon \beta}{2} e^{\frac{\beta}{2}\left(1-\epsilon^{2}\right)-\frac{\beta}{2} \tilde{f}\left(\epsilon^{2}, \frac{\beta}{2}\right)}\left(\left(1+\frac{\partial \tilde{f}}{\partial z}\left(\epsilon^{2}, \frac{\beta}{2}\right)\right)\left(1+\frac{\partial \tilde{f}}{\partial z}\left(\epsilon^{-2}, \frac{\epsilon^{2} \beta}{2}\right)\right)\right. \\
& \left.+\frac{2 \epsilon^{2}}{\beta} \frac{\partial^{2} \tilde{f}}{\partial z^{2}}\left(\epsilon^{2}, \frac{\beta}{2}\right)-\frac{\partial^{2} \tilde{f}}{\partial z \partial y}\left(\epsilon^{2}, \frac{\beta}{2}\right)\right) .
\end{aligned}
$$

As $\beta \rightarrow 0$, only one term on the rhs of Eq. 2.62 survives:

$$
p_{\beta=0}(\epsilon)=\epsilon^{3} \frac{\partial^{2} \tilde{f}}{\partial z^{2}}\left(\epsilon^{2}, 0\right) .
$$

Now this has to be a constant for $\epsilon$ inside the range $(\xi, 1 / \xi)$ (see Eq. (2.42) and App. A.4). As shown in App. A.3, this is the case if

$$
\tilde{f}(z, 0)= \begin{cases}1-z & \text { for } z \leq \xi^{2} \\ -\frac{4 \xi}{1-\xi^{2}} \sqrt{z}+\frac{1+\xi^{2}}{1-\xi^{2}}(z+1) & \text { for } \xi^{2}<z<\xi^{-2} \\ z-1 & \text { for } z \geq \xi^{-2}\end{cases}
$$

\subsubsection{Approximation for intermediate temperatures}

At intermediate temperatures, $0<y<\infty, \tilde{f}(z, y)$ shall be constructed in such a way that Eq. (2.64) is obtained in the limit $y \rightarrow 0$ and Eq. (2.60) holds in the limit $y \rightarrow \infty$. By writing

$$
\tilde{f}(z, y)=\sqrt{\tilde{f}^{2}(z, 0)+h(z, y)\left((1-z)^{2}-\tilde{f}^{2}(z, 0)\right)},
$$

where use has been made of yet another auxiliary function $h(z, y)$ which is invariant under the transformation $z \rightarrow 1 / z$ and $y \rightarrow z y$ (in order to satisfy the symmetry condition for $\tilde{f}(z, y))$, and such that $h(z, 0)=0$ and $0 \leq h(z, y) \leq 1$, the $y \rightarrow 0$ limit is automatically fulfilled. If $h(z, y)$ has the additional property

$$
\lim _{y \rightarrow \infty} h(z, y) \begin{cases}=1 \quad \text { if } z \leq \frac{p^{2}}{s^{2}} \text { or } z \geq \frac{s^{2}}{p^{2}} \\ <1 \quad \text { if } \frac{p^{2}}{s^{2}} \leq z \leq \frac{s^{2}}{p^{2}}\end{cases}
$$


and convergence is sufficiently fast, then also the low temperature limit Eq. (2.41) is satisfied (see Sec. 2.3.2 and Eq. (2.60)) since this condition ensures that

$$
\lim _{y \rightarrow \infty} \tilde{f}(z, y)\left\{\begin{array}{ll}
=|1-z| & \text { if } z \leq \frac{p^{2}}{s^{2}} \text { or } z \geq \frac{s^{2}}{p^{2}} \\
>|1-z| & \text { if } \frac{p^{2}}{s^{2}} \leq z \leq \frac{s^{2}}{p^{2}}
\end{array} .\right.
$$

Such a function is not hard to construct. One example is

$$
h(z, y)=1-\frac{v\left(1+v^{-1}\left(-a y \sqrt{z} \log \frac{z p^{2}}{s^{2}} \log \frac{z s^{2}}{p^{2}}\right)\right)}{v\left(1+v^{-1}(0)\right)+b y \sqrt{z}},
$$

where

$$
v(\eta)=\eta-\frac{1}{\eta} \quad \text { for } \eta>0
$$

and $v^{-1}(\eta)$ is the inverse function of $v(\eta)$. The parameters $a$ and $b$ can be used to fit the resulting expression for $p_{\beta}(\epsilon)$ to data from simulations (see Sec. 2.4). The full expression for $p_{\beta}(\epsilon)$ will not be presented here because it is very lengthy indeed. It can be generated, however, with e.g. the help of a symbolic algebra program by putting $h(z, y)$ from Eq. (2.68) into Eq. (2.65) and then applying Eq. (2.62).

There is one last thing that has to be checked, namely whether this particular choice of $h(z, y)$ is consistent with the condition $\partial^{2} f /(\partial x \partial y) \leq 0$. Although I am at present unable to prove this, numerical checks indicate that it holds.

\subsection{Comparison with computer simulations}

So far I have only dealt with analytic calculations. The approximations made, however, were rather uncontrolled. In order to test their quality, one must resort to computer simulations since the exact results are not available. Therefore I will first summarize very briefly the essential computational details (much more is to follow in the next chapter) before I make a comparison between the analytical and numerical probability densities.

\subsubsection{Computational details}

In order to obtain the probability distribution numerically, one has to go back to Eq. (2.18) and perform a Monte-Carlo simulation. The first thing to note is that for a given $q(\tau)$, Eq. (2.18) can easily be solved numerically by dividing the time axis in small intervalls and successively evaluating the max-function (which is then only the maximum of a finite number of values and depends only on previous, already known values of $w(\tau)$ ). This suggests the following algorithm for fixed $\beta$ : 
1. Randomly generate two Brownian bridge processes $q_{1}(\tau)$ and $q_{2}(\tau)$ with periods $\Gamma_{1}$ and $\Gamma_{2}$. This is simple since they are Gaussian, and their covariance is given by the respective terms in the sum on the rhs of Eq. (2.21) (this is the only point where $\beta=E_{\mathrm{tr}} / T_{\mathrm{B}}$ enters). Their sum is the process $q(\tau)$. See e.g. [KP92] for details on the generation of random processes on the computer.

2. Solve Eq. (2.18) numerically. Since the maximum collision time is known, it is only necessary to solve it up to this time (counted from the time of first contact).

3. Extract the value of $\epsilon$ from the final value of $w(\tau)$ according to Eq. (2.22) and insert it into a histogram.

4. Repeat from step 11 until the statistics are good enough.

This procedure has to be performed for each $\beta$-value that is of interest. The result is a histogram of $\epsilon$ for each $\beta$, such as it is shown in Fig. 2.2(a).

\subsubsection{Comparison of numerical and analytical probability densities}

Since the probability density that was constructed in the preceding section has only some (but certainly not all) of the properties of the real density, it must be compared with simulation results in order to check if the parameters $a$ and $b$ from Eq. (2.68) can be adjusted in such a way that the two densities agree. Fig. 2.2 demonstrates that for the particular case $\gamma=4 / 5, a$ and $b$ can indeed be nicely adjusted to match the simulation data. This is also true for other values of $\gamma$ which are not shown here. Although this comparison is not very precise, it is sufficient for the present purpose. It encourages the belief that the function constructed in this chapter captures the important aspects of $p_{\beta}(\epsilon)$. This view is also confirmed by the comparison made in the next chapter of many particle simulations performed with the numerical, "exact" distribution, and the new, approximate distribution.

\subsection{Summary}

The equations of motion for the two particle system with internal vibrations could be solved exactly, giving rise to an implicit equation containing memory terms, Eq. (2.18). If the initial conditions, i.e. the vibrational state of the rods before the collision, are unknown and are instead modelled by a random process, this results in a stochastic equation, giving rise to a fluctuating coefficient of restitution. The stochastic equation could be exploited to derive several exact properties of the transition probability $p_{\beta}(\epsilon)$ of the coefficient of restitution. These properties are rather 


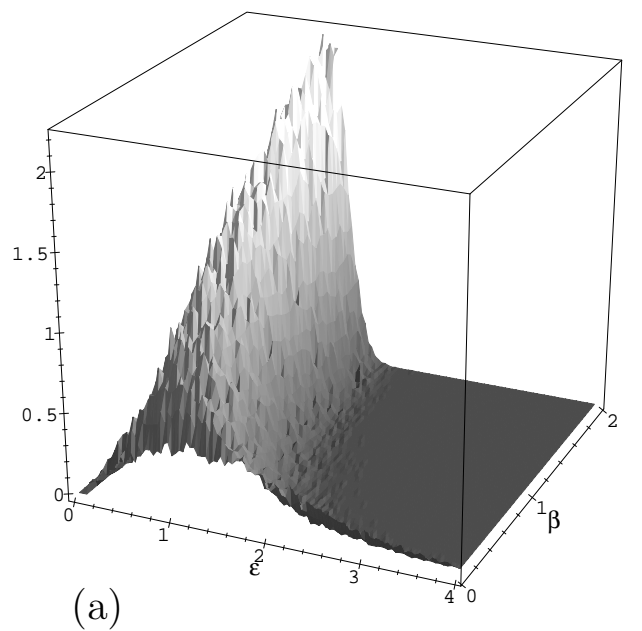

(a)

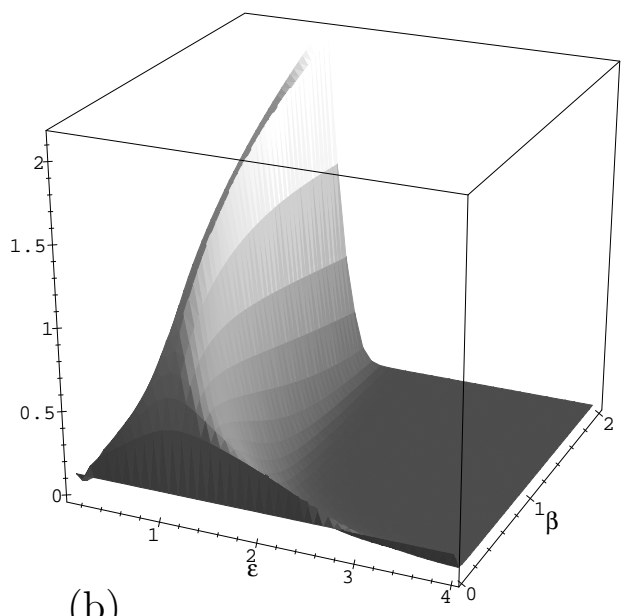

(b)

Figure 2.2.: (a) Simulation data for the probability distribution function for $\gamma=$ 4/5. (b) Analytic probability distribution function for $\gamma=4 / 5$. The parameters $a$ and $b$ of Eq. (2.68) were fitted to best match the data shown in (a). The agreement is quite satisfactory. Note that while it is possible to plot the $\beta=0$ limit in (b), it is impossible to reach this limit in the simulation (a). This is responsible for the apparent difference between the two graphs at $\beta=0$.

intriguing, in particular the extremely non-continuous dependence on the length ratio $\gamma$ as manifested e.g. in the upper and lower bounds. They could in turn be used to construct an analytical approximation which had the same properties and could nicely be fitted to numerical results. The relevance of this will become more evident in the next chapter where simulations using it will be discussed. Apart from this more technical use, a similar procedure as shown in this chapter can be used for the construction of other analytical expressions for $p_{\beta}(\epsilon)$, but still obeying some or all of the properties derived before, which might be used to test the universality of certain properties of the many particle system. 


\section{One-dimensional particles 2: Many particle systems}

After the presentation of the two particle model, the analytic derivation of exact properties of the transition probability $p_{\beta}(\epsilon)$, and the construction of an analytic approximation for it in the previous chapter, this chapter is devoted to numerical simulations of many particle systems using these results. While the simplest case is certainly the direct implementation of the model introduced in the previous chapter, it is not the most realistic because so far, the model conserves total energy. Yet the energy stored in vibrations must definitely be lost eventually, e.g. through black body radiation or by phonon scattering into regions of $k$-space that are irrelevant to the collision process. While these ideas do not fit into the general concept as outlined before (or would make it too complicated), it is possible to mimick their effect by introducing a relaxation time by which the vibrational energy stored in each vibrational mode is exponentially damped. Thus it is possible to simulate systems without total energy conservation. This makes a great difference in many respects, as will be shown below.

This chapter is therefore organised as follows: After a description of the details of the many particles system there follows a short introduction to the necessary computational prerequisites. Next, results from simulations using the "exact" method as described in Sec. 2.4.1 are presented. They illustrate the general behaviour of the system under consideration and its advantages over simulations of systems with a constant $\epsilon$, and they can also (at least in part) be compared to simulations using the approximate $p_{\beta}(\epsilon)$ in order to confirm the view that was already expressed in the previous chapter, namely that the approximation contains all essential features. Following this, several other simulation results of damped and undamped systems will be presented that were performed using the approximate but faster method.

\subsection{The many particle model}

The model for the individual particles and their interactions was presented in the previous chapter. The aim is now to simulate the dynamic evolution of $N$ such particles on a ring of circumference $L+\sum_{i=1}^{N} l_{i}$. $L$ is thus the total length of the interpar- 
ticle spacings. For the following arguments the actual lengths of the particles are unimportant because the point in time when a collision occurs depends solely on the end-to-end distance and the outcome of a collision depends only on the length ratio. In order to keep the notation simple the system is therefore mapped to an equivalent one consisting of $N$ point particles on a ring of circumference $L$. Each particle is characterized by its position $R_{i}(t)$, its velocity $\dot{R}_{i}(t)$, and the temperature of its internal bath $T_{\mathrm{B}}^{(i)}(t)$. The $N^{\text {mod }}$ ( $N^{\text {mod }}$ being the same for all rods) internal modes of a rod are represented by one degree of freedom only, namely $T_{\mathrm{B}}^{(i)}=\sum_{\nu=1}^{N^{\text {mod }}} E_{i \nu} / N^{\text {mod }}$, where $E_{i \nu}$ is the vibrational energy of a mode. The rods are assigned alternating lengths such that the ratio $\gamma$ for each collision has a fixed value, in this case $4 / 5$. The ratio of masses is also given by $\gamma$, assuming the same homogeneous mass density for both kinds of rods. The rods are assigned alternating lengths because due to the result given in Sec. 2.2.5, a length ratio of $\gamma=1$ implies $\epsilon=1$ always which would simply correspond to a standard one dimensional hard sphere gas.

\subsection{Computational methods}

In order to simulate such a many particle system, some simplifications have to be made:

- It is assumed that two particle collisions dominate the dynamic evolution of the system. This is justified for a dilute granular gas. The typical time of interaction in the model is given by $t_{\text {int }}=2 l / c$, i.e. the time a signal needs to travel back and forth on a rod. Hence in principle, two colliding rods can interact with a third one. This will be highly unlikely, as long as the time between collisions is much longer than $t_{\text {int }}$. This requires $2 l / c \ll L /\left(N\left|\dot{R}_{i+1}-\dot{R}_{i}\right|\right)$. So either the length of the rods has to be chosen sufficiently small as compared to the mean distance $L / N$ or the initial velocities $\left|\dot{R}_{i+1}-\dot{R}_{i}\right|$ should be small compared to the velocity of sound. The latter is a material parameter and can have quite high values for hard materials (e.g. for steel, $c \sim 10^{4} \mathrm{~m} / \mathrm{s}$ ), favouring short interaction times. In a standard event-driven simulation inelastic collapse (see Sec. 3.2.2) occurs when the number of particles is sufficiently large, resulting in a diverging collision frequency. This would clearly violate the condition that the time between two collisions is long compared to $t_{\text {int }}$. However, since the algorithm avoids the inelastic collapse, as will be discussed below, I will still make use of the assumption that three or more particle collisions will not be important. The whole system will thus be described by instantaneous two particle collisions with a stochastic coefficient of restitution $\epsilon$.

- Next, there are two ways to generate the random coefficient of restitution $\epsilon$ : The first is to solve Eq. (2.18) numerically, as was described in Sec. 2.4.1, the second is to use the approximate $p_{\beta}(\epsilon)$. While the former is exact (at least 
apart from numerical errors), the latter is not. However, it is by at least a factor of 10 faster than the former which is the main reason for its use.

- Another approximation concerns the fact that, in principle, the two particles participating in a collision may have different internal bath temperatures. This does not pose a problem when using the first method to generate $\epsilon$, but it does for the approximate method since $p_{\beta}(\epsilon)$ is only known for equal bath temperatures. Therefore, before performing a collision, an average bath temperature is computed from the two particles and the corresponding $\beta$ is used for the collision. Additionally, neither of the two methods can determine how the vibrational energy of the two particles is distributed between themselves after the collision. For simplicity, they are both assigned the same amount.

\subsubsection{The Event driven/Monte Carlo algorithm}

The algorithm that will be used is a hybrid of an event driven algorithm and a Monte Carlo simulation. The particles move freely in between collisions, as in event driven algorithms. When two particles collide their states are updated stochastically, according to the distribution of $\epsilon$.

It is convenient to introduce dimensionless variables $x_{i}=R_{i} N / L$ and $v_{i}=$ $\dot{R}_{i} \sqrt{\mu / T_{0}}$. $\quad T_{0}$ serves as an energy scale and will be identified with the homogeneous initial granular temperature of the many particle system. Time is measured in units of $L \sqrt{\mu / T_{0}} / N$.

For the algorithm one only needs relative distances and velocities

$$
\begin{aligned}
& \Delta x_{i}=\left\{\begin{aligned}
x_{i+1}-x_{i} & \text { for } 1 \leq i \leq N-1 \\
N+x_{1}-x_{N} & \text { for } i=N
\end{aligned}\right. \\
& \Delta v_{i}=\left\{\begin{array}{c}
v_{i+1}-v_{i} \text { for } 1 \leq i \leq N-1 \\
v_{1}-v_{N} \text { for } i=N
\end{array}\right.
\end{aligned}
$$

The algorithm is defined by iteration of the following steps:

1. Calculate the time difference $\Delta t$ for the next collision to take place:

$$
\Delta t=\min _{\left\{i \mid \Delta v_{i}<0\right\}}\left(-\frac{\Delta x_{i}}{\Delta v_{i}}\right)
$$

The pair of particles which is going to collide next is denoted by $\left(i_{0}, i_{0}+1\right)$.

2. The relative distances of all particles are updated according to:

$$
\Delta x_{i}(t+\Delta t)=\Delta x_{i}(t)+\Delta v_{i}(t) \Delta t
$$

For the designated pair $\left(i_{0}, i_{0}+1\right)$ one obtains $\Delta x_{i_{0}}(t+\Delta t)=0$. 
3. The kinetic energy of relative motion of the pair $\left(i_{0}, i_{0}+1\right)$ as well as the mean local bath temperature are calculated according to $E_{t r}=\Delta v_{i_{0}}^{2} / 2$ and $T_{B}=\left(T_{B}^{\left(i_{0}\right)}+T_{B}^{\left(i_{0}+1\right)}\right) / 2$. Subsequently, a random value of $\epsilon$ is chosen from the probability distribution $p_{\beta}(\epsilon)$, either by numerically solving Eq. (2.18) and applying Eq. (2.22) or by using the analytical form for $p_{\beta}(\epsilon)$. See Sec. 2.4.1 for a detailed description of the former and Sec. 3.2.3 for the latter method.

4. The bath temperatures and relative velocities are updated

$$
\begin{aligned}
T_{B}^{\left(i_{0}\right)}(t+\Delta t) & =T_{B}^{\left(i_{0}+1\right)}(t+\Delta t) \\
& =T_{B}+\frac{1-\epsilon^{2}}{2 N^{\text {mod }}} E_{t r} \\
\Delta v_{i_{0}-1}(t+\Delta t) & =\Delta v_{i_{0}-1}(t)+\frac{1+\epsilon}{2} \Delta v_{i_{0}}(t) \\
\Delta v_{i_{0}}(t+\Delta t) & =-\epsilon \Delta v_{i_{0}}(t) \\
\Delta v_{i_{0}+1}(t+\Delta t) & =\Delta v_{i_{0}+1}(t)+\frac{1+\epsilon}{2} \Delta v_{i_{0}}(t) .
\end{aligned}
$$

\section{Continue with step 11.}

This algorithm is not the most efficient one conceivable; Lubachevsky [Lub.91] introduced a better method. However, the algorithm above is simple and still only $\mathcal{O}(N)$ (as the one from [Lub.91]) because the search for the next collision partner is trivial in one dimension.

\subsubsection{Inelastic collapse}

One of the benefits of the model under consideration is that it avoids the so called "inelastic collapse". This section is therefore not concerned with problems of the present work but rather with problems of other methods and why they do not appear here.

The inelastic collapse has been widely discussed in the literature [BM.90, MY.92, MY93, CSB98, GSB ${ }^{+}$98, SB99, CDKK99, OU98, LM98]. In short, it describes the phenomenon that inelastically colliding particles can experience an infinite number of collisions in a finite time (in the idealised situation that the duration of a collision is zero, i.e. if the collisions are instantaneous), thus losing all of their kinetic energy in finite time. While this is quite natural experimentally (e.g. a handful of sand comes to a complete stop after a short time when dropped on the floor) and is a very interesting phenomenon in its own right, it is more of a nuisance for numerical simulations. If collisions between particles are handled one after the other (as in the standard event driven method), the simulation will obviously never get beyond the point in time where the collapse occurs since this would involve infinitely many collision events. Therefore one either has to restrict oneself to small numbers of 
particles and coefficients of restitution close to 1 where the collapse does not occur (the so-called quasielastic limit [MY92, MY93] ) or to make use of modified collision rules that avoid the collapse. One example for the latter case is the TC-model [LM.98] where particles that have already suffered a collision a very short time ago are assigned a coefficient of restitution $\epsilon=1$ for their next collision. Another example is the method proposed in [DB.97] where rebound velocities are rotated by a small random angle after each collision. Obviously, this does not work in one dimension. What's more, both methods require the input of some more or less ad hoc parameters.

The collision model of the present work intrinsically shows no inelastic collapse for the following reasons: When an inelastic collapse is at hand, i.e. when three or more of the elastically deformable particles start to collide in rapid succession with each other, their internal modes will become excited. When they have been excited, there is a finite probability that the outcome of a collision is not inelastic but (possibly even over-) elastic $(\epsilon \geq 1)$. This probability increases with the degree of excitation (see Fig. 2.2 for details on the transition probability). Therefore such situations will be broken up by the energy stored in the internal modes. This is even the case if the internal vibrations are damped (as will be the case later on) since the time between collisions will eventually become so short that damping is negligible.

\subsubsection{Implementation of a random number generator}

In order to make use of the analytical approximation calculated in the previous chapter, one has to implement a random number generator that generates random numbers distributed according to this probability density. To this end, the inverse of the distribution function $D(x, y)$ is needed (see e.g. [PTVF92], Ch. 7). The distribution function can be computed from Eq. (2.52):

$$
D(x, y)=\int_{0}^{x} p\left(x^{\prime}, y\right) d x^{\prime}=1-\frac{1}{2} e^{y-x-f(x, y)}\left(1+\frac{\partial f}{\partial y}(x, y)\right)
$$

This can be transformed into the distribution function for $\epsilon$ and $\beta$ :

$$
D_{\beta}(\epsilon)=1-\frac{1}{2} e^{\frac{\beta}{2}\left(1-\epsilon^{2}\right)-\frac{\beta}{2} \tilde{f}\left(\epsilon^{2}, \frac{\beta}{2}\right)}\left(1+\frac{\partial \tilde{f}}{\partial z}\left(\epsilon^{-2}, \frac{\epsilon^{2} \beta}{2}\right)\right)
$$

where $f(x, y)$ has again been expressed in terms of $\tilde{f}(z, y)$ as in Eq. (2.61).

It is probably impossible to calculate the inverse of this function analytically but it is easy to invert numerically [PTVF.92]. The random $\epsilon$ are finally generated by plugging a uniformly distributed random number $r$ from the interval $(0,1)$ into $D_{\beta}^{-1}$ :

$$
\epsilon=D_{\beta}^{-1}(r)
$$




\subsection{Simulation results using the "exact" method}

In this section, simulations using a numerical solution of Eq. (2.18) at every collision event will be presented. The simulations were performed on a many particle system with 10000 particles. Where necessary, the results for this large system are supplemented by runs of a 50 particle system for illustrational purposes. At the beginning of each simulation run, the system was prepared in a homogeneous state and all internal bath temperatures were set to zero. Thus the cooling behaviour, i.e. the transfer of translational energy into the internal baths, could be studied as time evolves. The data will first be analysed with regards to global properties (total kinetic energy, collision rates). Second, local quanties such as particle and energy densities will be considered.

\subsubsection{Global quantities}

\section{Kinetic energy}

At the beginning of a simulation run, the system is prepared in a homogeneous state and all internal bath temperatures are set to zero. Then the decay of the kinetic energy is monitored.

The time development of the total kinetic energy, which is given by

$$
E_{\mathrm{kin}}=\sum_{i=1}^{N} \frac{\Gamma_{i}}{2} v_{i}^{2}
$$

in dimensionless units, is shown in Fig. 3.1, in comparison with results for the deterministic model with constant $\epsilon$. For small times the curves for the deterministic and the stochastic dynamics are similar. In the initial stage very little energy is stored in the internal modes and hence the coefficient of restitution is approximately given by the deterministic value. The deterministic system, however, runs quickly into the inelastic collapse, as can be seen from the total number of collisions which is shown as a function of time in Fig. 3.2. When this happens, the simulation gets stuck so that the curve for the kinetic energy breaks off in Fig. 3.1. The stochastic dynamics shows completely different behaviour: The kinetic energy continues to decrease until equilibrium is reached, where $E_{\text {kin }}$ continues to fluctuate around the stationary value which is given by $E_{\text {kin }}^{\text {stat }}=E_{\text {kin }}(t=0) /\left(2 N^{\text {mod }}+1\right)$. The final state has not yet been reached for the 10000 particle run in the time interval that is shown in Fig. 3.1. For this reason, the results of a simulation run with 50 particles have been included in the figure to show that equilibrium is reached eventually and that equipartition holds in the stationary state. There are considerable fluctuations in the curves for the kinetic energy in Fig. 3.2 (around $t=10^{4}-10^{5}$ ) before equilibrium is reached: these are due to the formation and breakup of clusters as will be shown below. The final state of the stochastic model is a consequence of the idealised assumption that 


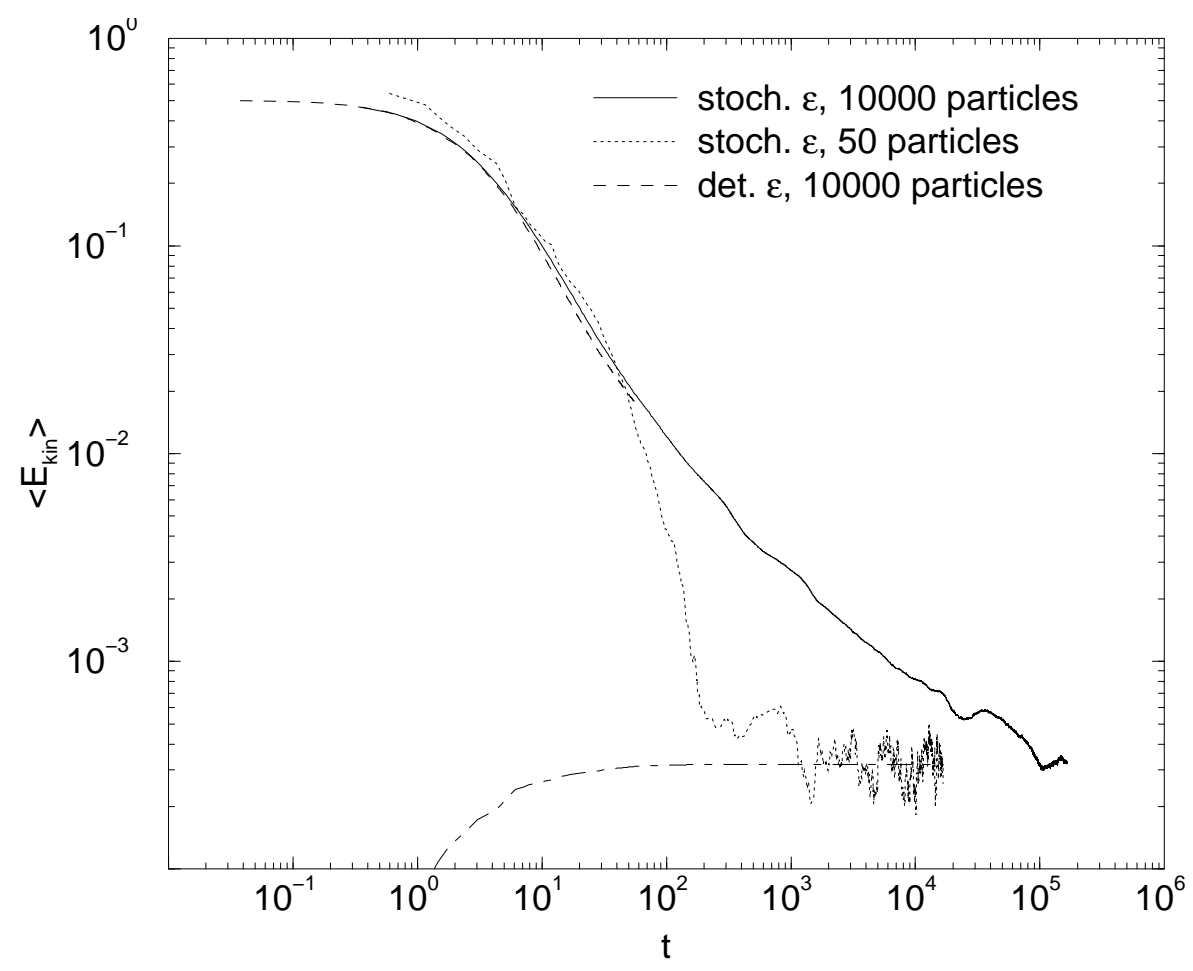

Figure 3.1.: Reduction of the kinetic energy per particle as a function of time. The curve for the deterministic coefficient of restitution breaks off because an inelastic collapse occured. The dash-dotted line shows the average energy per vibrational mode for the 50 particle run and illustrates that equipartition holds in the stationary state.

the total system be conservative. As demonstrated below, this changes when net energy dissipation is included in the model.

\section{Collision rate}

Simple mean field arguments [Haf8.3, SG9.5] have been used to derive scaling laws for the time evolution of kinetic energy and collision rate. One assumes that the particle velocities are uncorrelated and Gaussian distributed. For a constant coefficient of restitution one obtains $E_{\text {kin }}(t) \sim t^{-2}$ and $\dot{N}_{\text {coll }} \sim \ln t$. Neither scaling law fits the data here, as can be seen from figs. 3.1 and 3.2. McNamara and Young [MY.93] have already pointed out that the mean field scaling laws are only applicable in the quasi elastic regime, where no inelastic collapse occurs. Otherwise the assumption of uncorrelated Gaussian velocities breaks down. In the stochastic model one has additional fluctuations of the coefficient of restitution, which invalidate the derivation of the above scaling laws. Hence it is no surprise that the data disagree with these 


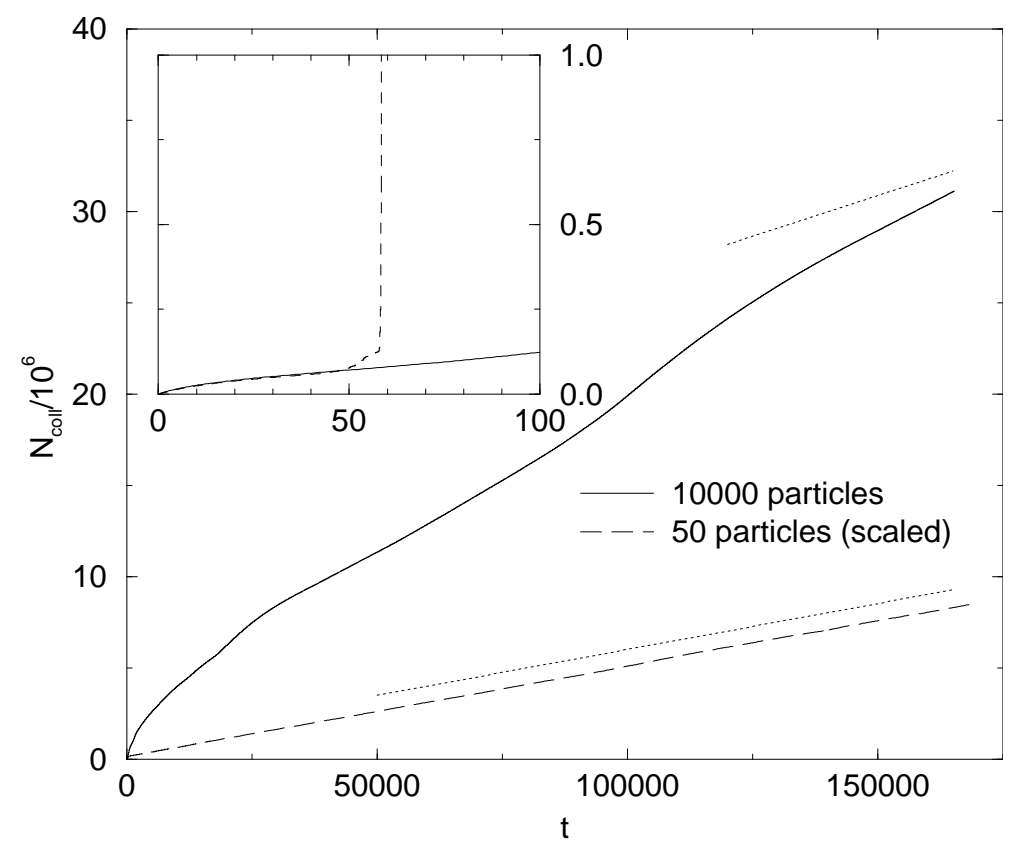

Figure 3.2.: Number of collisions as a function of time. The inset shows a comparison of the deterministic model (dashed line) and the stochastic model (solid line). (The units on the axes of the inset are the same as on the regular axes.) The deterministic model quickly runs into the inelastic collapse, seen by the diverging number of collisions. The dotted lines shows the theoretical number of collisions as a function of time in the stationary state according to Eq. (3.14) for the 10000 and the 50 particle runs. The data for the 50 particle run has been scaled by a factor of 100 in order to fit on the graph.

relations.

The rate of collisions becomes constant as the stationary state is approached, as can be seen from Fig. 3.2. The average collision rate is given by $\dot{N}_{\text {coll }}=N \overline{\Delta v} /(2 \overline{\Delta x})$. In the stationary state the velocities are indeed uncorrelated Gaussian variables, distributed according to

$$
p_{j}(v)=\left(\frac{2 \pi}{2 N^{\text {mod }}+1}\right)^{-1 / 2} \exp \left(-\frac{\Gamma_{j} v^{2}}{2\left(2 N^{\text {mod }}+1\right)^{-1}}\right),
$$

where $j=1(2)$ again stands for the shorter (longer) type of rods. Assuming $\overline{\Delta x}=1$ and performing the average over velocities, one obtains

$$
\dot{N}_{\text {coll }}^{\text {stat }}=\frac{N}{2 \sqrt{\pi\left(2 N^{\text {mod }}+1\right)}} .
$$


This result is in very good agreement with the simulations of the 50 particle system in the stationary state (see Fig. 3.2). The 10000 particle system is also approaching the correct value as it gets closer to the stationary state.

\subsubsection{Local quantities}

Apart from the global observables which characterise the system as a whole, much information about the system and its dynamics is contained in local quantities like density fields. In the following, several such quantities will be analysed in order to illuminate the behaviour of the system under consideration.

\section{Particle density}

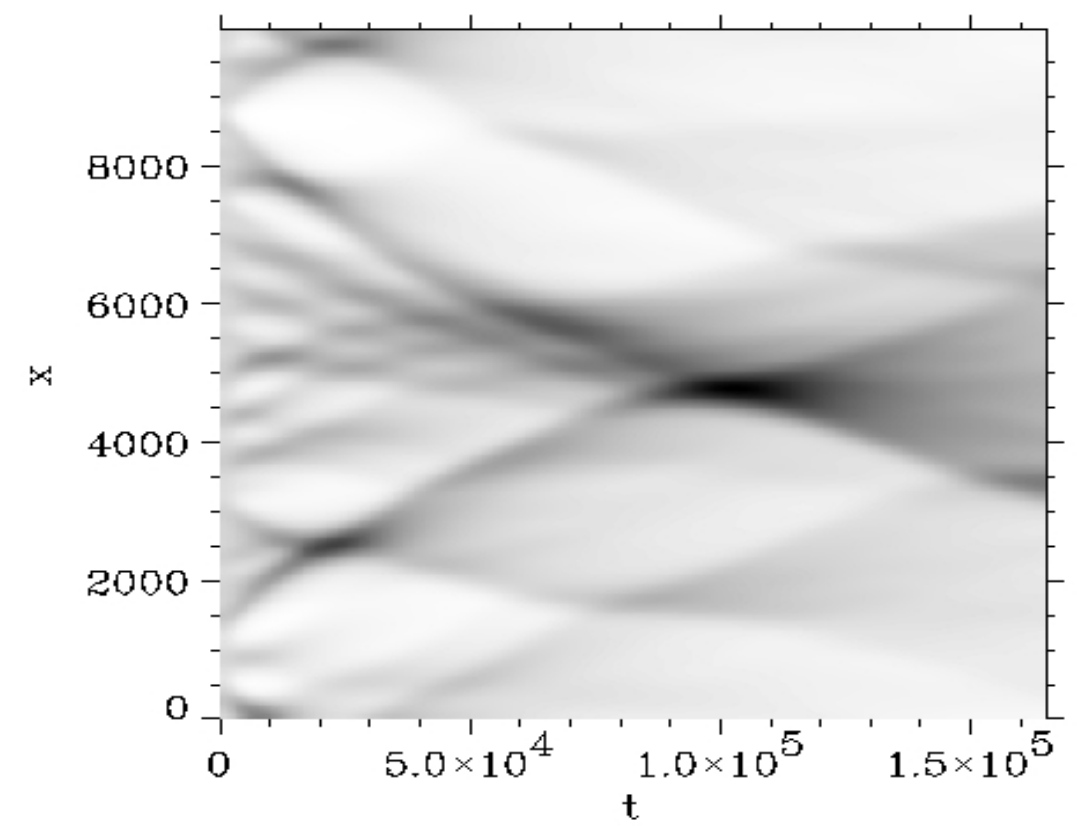

Figure 3.3.: Time evolution of the coarse grained particle density. Dark (bright) regions indicate high (low) density.

The system is initialised again with a spatially homogeneous distribution of particles. Evolving spatial structures will be analysed with help of a coarse grained density $\rho$. The total length of the ring is divided into 100 bins and the number of particles in each bin is counted. The coarse grained density is defined as the actual number of particles in each bin divided by the average. 
The time evolution of $\rho$ is shown in Fig. 3.3. Several phases in the cooling process can be identified. First, the particles start to form clusters and voids as they lose kinetic energy in collisions (initially, when $T_{\mathrm{B}}$ is small compared to the translational energy, the coefficient of restitution is always close to $\gamma$ ). After these clusters have formed, one observes collisions of clusters, forming larger clusters. Simultaneously the dissolution of clusters starts to set in, the remains being sent outwards to join neighbouring clusters. The biggest clusters and voids are seen to survive for times of order $10^{4}$. A similar observation has been made by Puglisi et. al. [PLM ${ }^{+}$98] who examined a one-dimensional system of Brownian particles with inelastic collision rules. In contrast to the present system, however, they found a steady state in which clusters are continously created and destroyed, while here the creation and destruction of clusters is only an intermediate phase of the cooling process: The complex interaction of forming and dissolving clusters continues with a clear tendency to form fewer and larger clusters. However, the actual approach to equilibrium is not accessible with the slow simulation method used in this chapter. It is therefore necessary to use the faster method using $p_{\beta}(\epsilon)$, the results of which will be shown in Secs. 3.4 and 3.5 .

The formation and break-up of clusters is responsible for the fluctuations in the energy decay as shown in Fig. 3.1: When two clusters collide to form a larger one, much of the translational energy of the merging clusters is temporarily transferred into vibrational energy, resulting in one of the dips in Fig. 3.1. When later the bigger cluster breaks up again, the vibrational energy is retransformed in translational energy, hence the maybe unexpected increase in kinetic energy at the end of a dip.

For 10000 particles it takes a time longer than the simulation range until the cooling dynamics is finished and the equilibrium state is reached, whereas for 50 particles it takes only a time of order $10^{3}$ (see Fig. 3.1). It will be shown below that the time to reach equilibrium for the 10000 particle system is in fact exceedingly long.

\section{Phase space}

The complete information about the state of the system at time $t$ is contained in a phase space plot, as shown in Fig. 3.4. Within a cluster of particles one expects frequent collisions and hence an effective transfer of kinetic energy to internal vibrations. Frequently, regions of high average density are characterized by particle velocities centered around zero. However, one also observes clusters with an average nonzero velocity, resulting at a later time in collisions of clusters. One such collision of two clusters can be traced in Fig. 3.4 around $x \sim 2500$. In Fig. 3.4(a) (a snapshot taken at $t=14000$ ) one observes two clusters both with nonzero average velocity moving towards each other, wheras in Fig. 3.4(b) (taken at $t=20000$ ) the clusters have collided and formed a larger one.

One also sees around $x \sim 1000$ the occurrence of a stripe-shaped fluctuation in the 

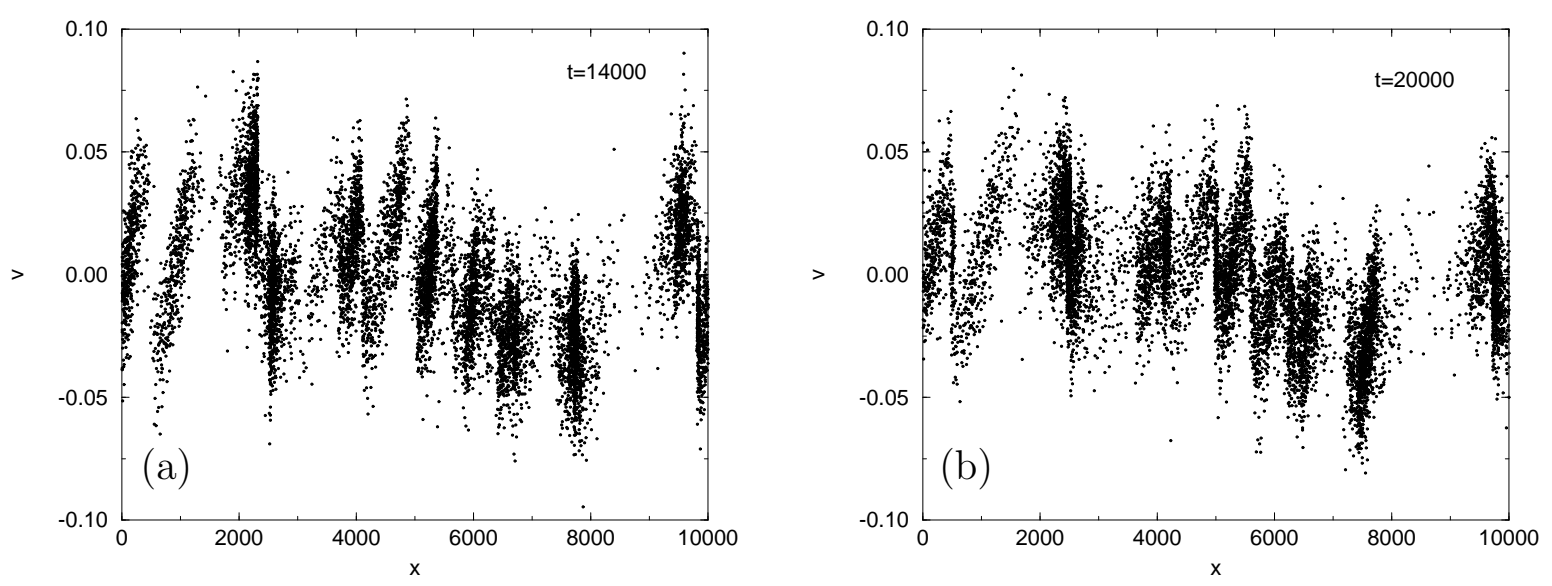

Figure 3.4.: Phase space plot of the system at two different times.

phase-space plot. This type of fluctuation has already been observed and discussed by McNamara and Young [MY93] and Sela and Goldhirsch [SG.95]. It gives rise to the formation of clusters out of an initially homogeneous region. Thus figure 3.4 shows that the dynamics of the present system are indeed rather complex as formation, movement, interaction and dissolution of clusters all happen simultaneously.

\section{Local kinetic energy}

It is interesting to see how the kinetic energy is spatially distributed. A coarse grained kinetic energy density is defined similar to the coarse grained density by summing the kinetic energies of all particles inside a bin and dividing by the number of particles in the bin.

One might be tempted to conjecture that the local kinetic energy is in some way correlated to the clustering because most collisions occur within the clusters. Fig. 3.5 (as an example) reveals, however, that this is generally not the case: although the kinetic energy shows some structure there is no visible correlation to the density, not even in a state as the one shown in Fig. 3.5, where all the particles are extremely clustered. Fig. 3.5 is a snapshot of the system at time $t=100343$ (cf. Fig. 3.3).

\section{Velocity distribution}

In the cooling stage, the system is still far from equilibrium, so that the velocity distribution of the particles is not expected to be a Maxwell distribution. It is therefore interesting to test what kind of distribution the velocities really follow.

Data analysis shows that the velocity distribution of all particles is indeed not a Gaussian distribution (see Fig. 3.6). There are relatively large systematic deviations 


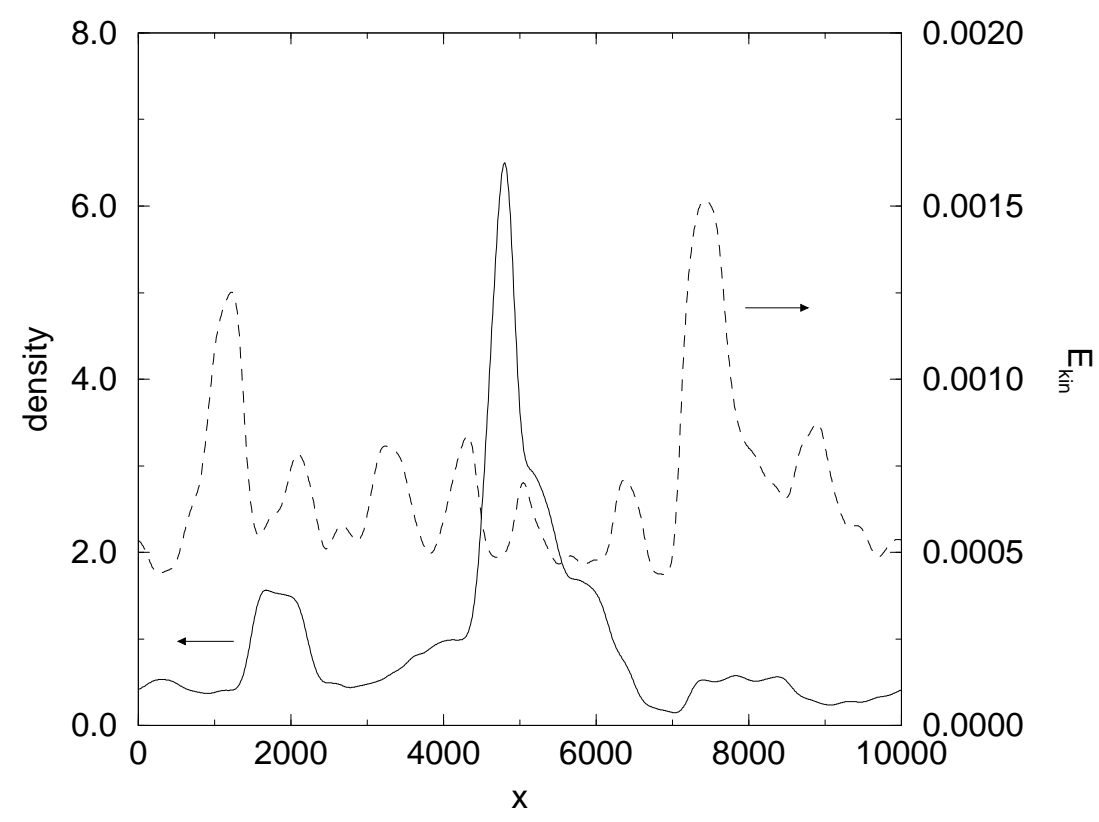

Figure 3.5.: Comparison of the local kinetic energy (dashed line) and particle density (solid line).

especially near the maximum of the curve. If one restricts the data analysis to only those particles inside a single cluster, however, one finds that the velocity distribution of these is to a much better degree gaussian, considering that there are only about $1 / 10$ th of the total number of particles in the cluster. This is not surprising, however, since there are many collisions between particles inside a cluster and thus a local equilibrium is reached, resulting in a Maxwellian distribution. On the other hand the velocity distribution of all particles reflects the velocity distribution of the clusters. As long as the complicated process of forming and dissolution of clusters is underway, the clusters are naturally far from equilibrium. This leads to the observed deviations from the gaussian curve.

Since the system is far away from the quasielastic limit, one sees quite a different velocity distribution than MacNamara and Young [MY93], who simulated a one dimensional system of quasielastic particles. They observed a bimodal velocity distribution because the particles tend to concentrate on the upper and lower edges of a band in a phase space plot similar to Fig. 3.4. In this simulation, the situation is much more complex because of the formation of many clusters, each with its own velocity distribution. 


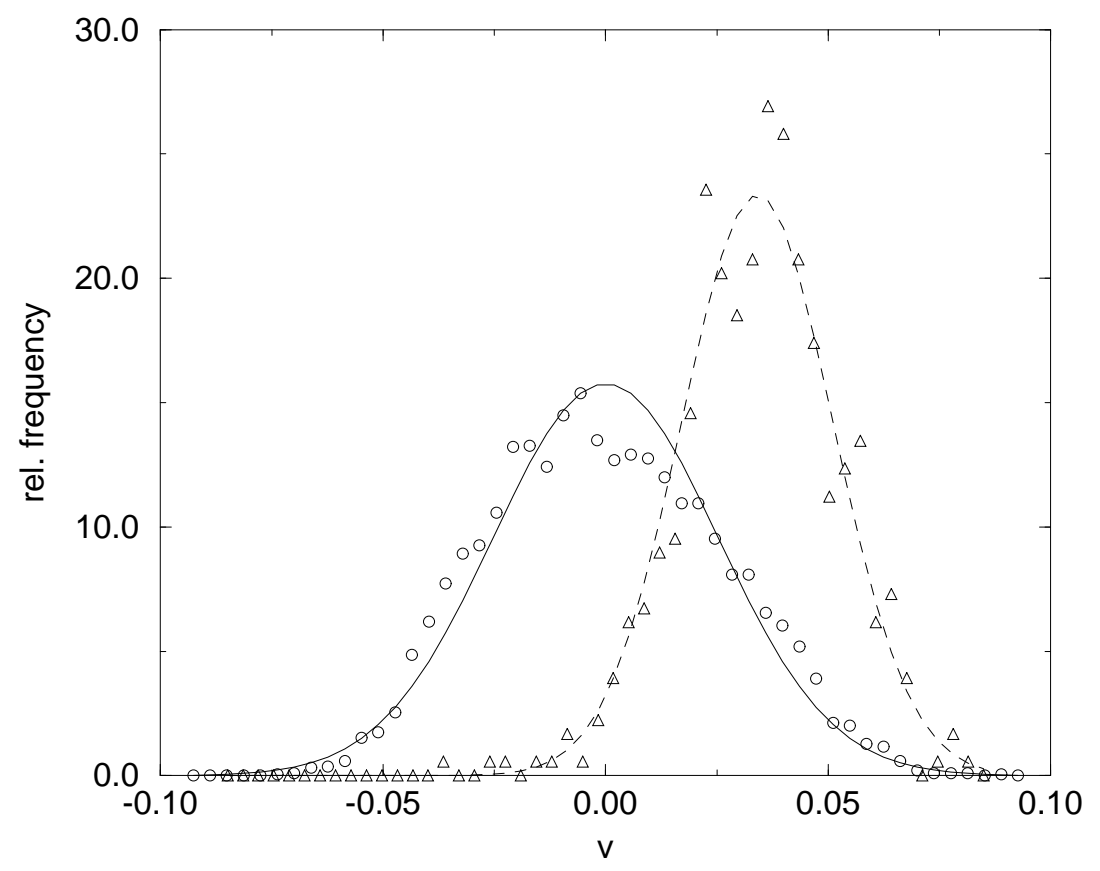

Figure 3.6.: Velocity distribution of all particles (circles) and the particles inside one particular cluster (triangles) at time $t=14000$. The cluster chosen for this curve is centered around $x=2000$ (cf. Figs. 3.3 and 3.4(a))

\section{Correlation function}

The inelasticity of collisions leads to a clustering of particles, as can be seen in Fig. 3.3. Williams [Wil96, WM96] has described a one dimensional system of individually heated granular particles. He found that the pair correlation function, defined by $g(x)=\frac{1}{N-1} \sum_{i \neq j} \delta\left(x-\left|x_{i}-x_{j}\right|\right)$ of the system in the steady state approximately follows a power law. Here, one observes quite a different behaviour of the correlation function (see Fig. 3.7). Instead of showing a divergence at zero separation, it levels off to a plateau. The explanation for such a different behaviour lies in the mechanism of heating: When the particles are heated individually, i.e. when they are driven by a random force, they will half of the time be kicked back in the direction of the particle that they last collided with. Thus there is some additional tendency for the particles to stick together. In this model, however, the particles will only change their velocity when they collide, thus favouring larger distances.

It should be noted that the correlation function in Fig. 3.7 is not that of the steady state of the system but a snapshot taken during the cooling process. 


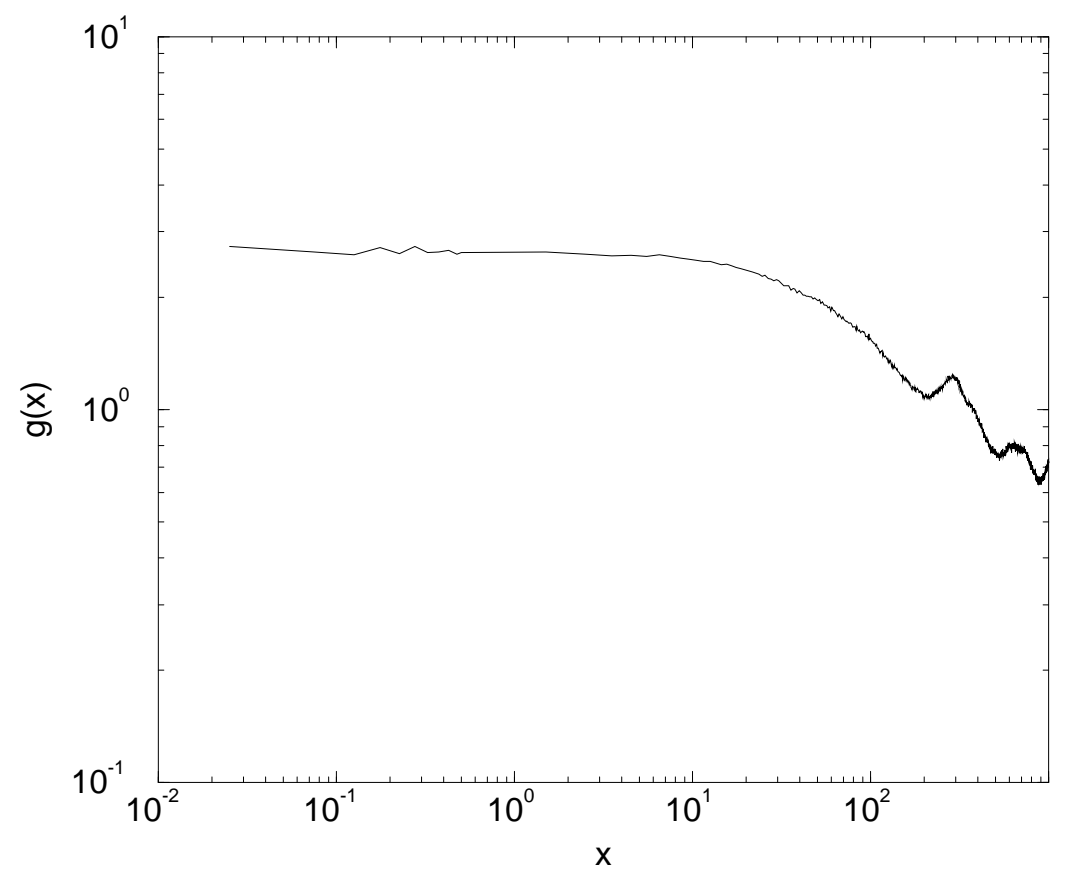

Figure 3.7.: The pair correlation function $g(x)$ of the system at $t=14000$.

\subsection{Comparison of many particle simulations using the numerical and analytical $p_{\beta}(\epsilon)$}

In order to estimate the quality of the approximation for $p_{\beta}(\epsilon)$, runs of systems with 10000 particles using both the "exact" method and $p_{\beta}(\epsilon)$ were performed. Again, the length ratio $\gamma=4 / 5$ was used. The time decays of the translational energy for both runs are compared, Fig. 3.8 shows the result. The curves agree very well, keeping in mind that they are merely single runs which necessarily show fluctuations. These fluctuations are due to production and breakup of clusters (see Secs. 3.3.1 and 3.3.2). Fig. 3.9 shows the time evolution of the density in a similar plot as Fig. 3.3. Note the similarities between these two graphs, but note also the difference in time scales which is due to the increased simulation speed which makes longer runs possible. This agreement supports the claim that the approximated probability distribution captures the essential features of the model.

The road to equilibrium as seen in this simulation confirmes the remarks made earlier: The system evolves first by creating many small clusters, which succesively collide to form fewer and larger ones. But since here the simulation time is about one decade longer than in the run shown before, some more details can be inferred 


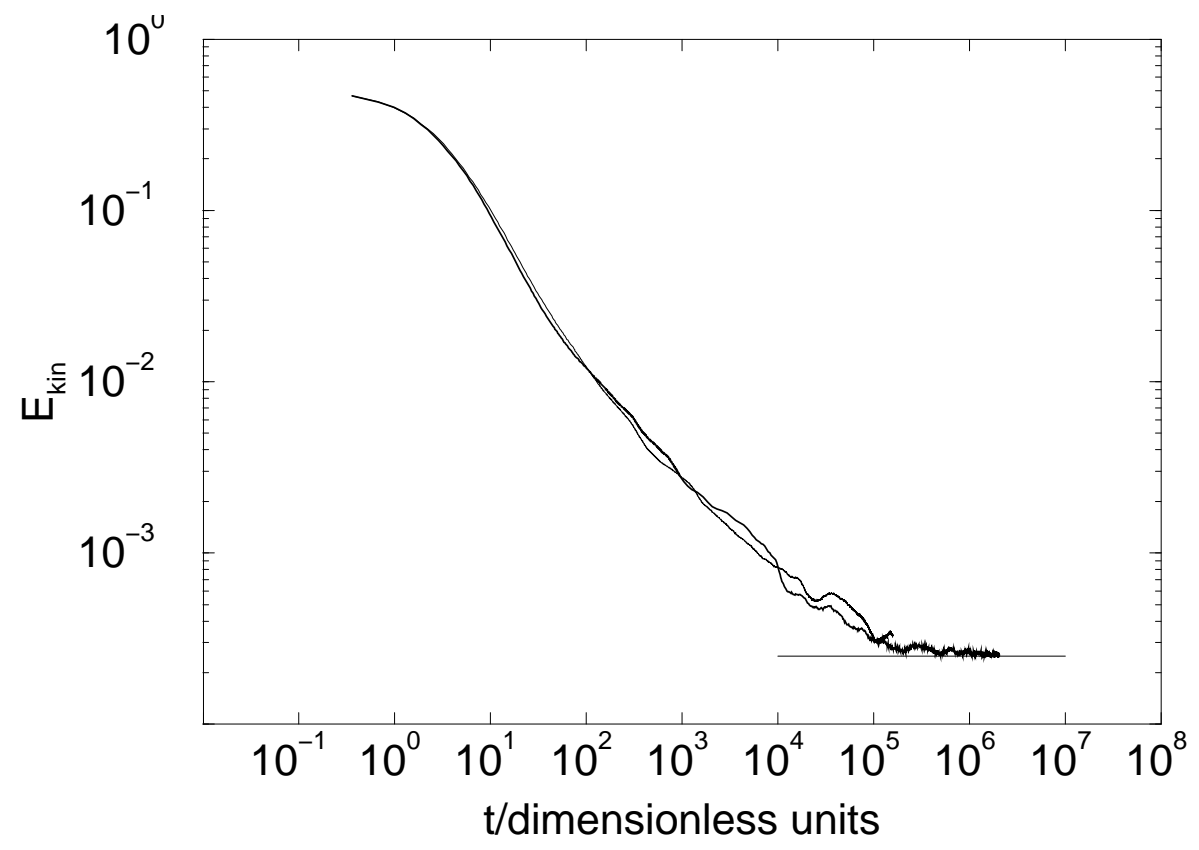

Figure 3.8.: Energy decay towards equilibrium using the "exact" method (thin line) and using $p_{\beta}(\epsilon)$ (thick line). The agreement is very good. The equilibrium value of the energy is indicated by the horizontal line. Note that due to increased simulation speed the thick line extends more than one decade further than the thin line.

from Fig. 3.9: The larger clusters are becoming ever less distinct; towards the end they are merely small density fluctuations which travel back and forth.

Note also that Fig. 3.9 shows that the approach to the true equilibrium state is extremely slow: Even though the kinetic energy has decayed to basically its equilibrium value (Fig. 3.8), there are still visible correlations in the density. The explanation for this phenomenon will be given in the next section.

\subsection{Application of $p_{\beta}(\epsilon)$ to many particle systems}

Since the previous section has shown that the two simulation methods give identical results, I will now concentrate on using the faster of the two in order to be able to perform longer runs of larger systems. The results of these simulations are presented in this section. 


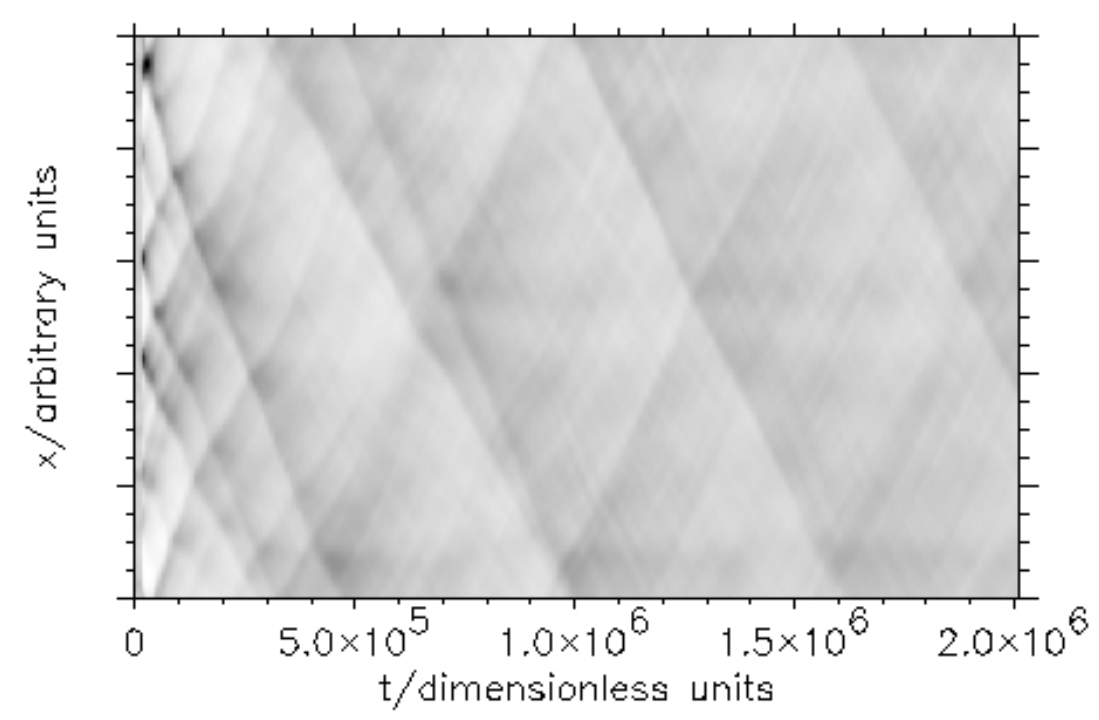

Figure 3.9.: Time evolution of the particle density from a simulation using the approximate $p_{\beta}(\epsilon)$ starting from a nonequilibrium state with all internal bath temperatures set to 0 . The corresponding decay of the kinetic energy is shown in Fig. 3.8. Grayscale coding is as described in the caption to Fig. 3.3.

\subsubsection{Undamped equilibrium system}

As a first application I consider a system of 10000 particles on a ring in equilibrium, i.e. with all internal bath temperatures set to such an initial value that equipartition of the energy over all modes, translational and vibrational, is given. Naively, it could be argued that this state (as it is an equilibrium system of particles coupled to the heat bath of internal oscillators) is trivial and identical to a one-dimensional gas of completely elastic point particles. The simulations show that this is not the case; Fig. 3.11 shows a comparison of the dynamical structure factors $S(k, \omega)$ of an gas of elastic particles and of the aforementioned simulation. While the dynamic structure factor for the ideal gas is essentially structureless, the particles with internal modes show a distinct Brioullin line (see e.g. [HM86, Ch. 8]) corresponding to soundwave propagation. This can also be verified by eye in Fig. 3.10 where a certain preferred slope in the time developement of the density is evident. The absence of a Rayleigh line centered around $\omega=0$ in the dynamic structure factor is due to the fact that the prefactor of this line is given by $\left(c_{p}-c_{v}\right) / c_{p}$ where $c_{p}$ and $c_{v}$ are the specific heats per particle at constant pressure and volume, respectively [HM86]. In the present case, each particle is equipped with a large number of internal modes, each of which 


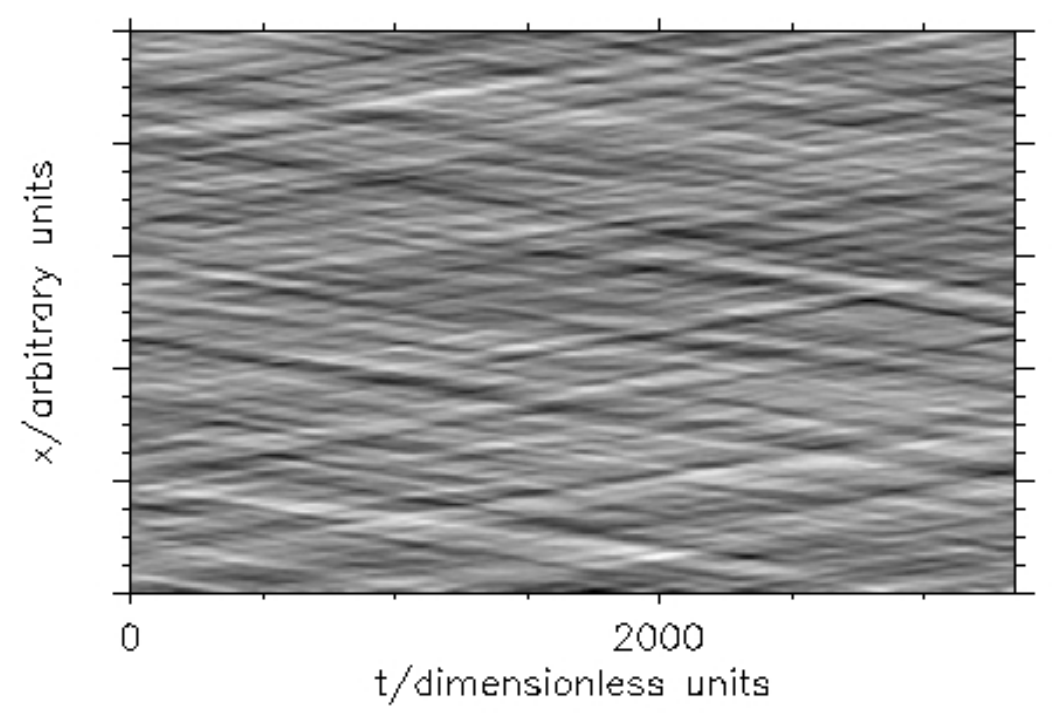

Figure 3.10.: Time evolution of the coarse grained spatial density of a system of 10000 particles using $p_{\beta}(\epsilon)$. Stripes with a characteristic slope can be identified, i.e. density fluctuations travel with a certain velocity; this corresponds to soundwave propagation as described in detail in Sec. 3.5.

contributes $k_{\mathrm{B}}$ (Boltzmann's constant) to both $c_{p}$ and $c_{v}$. This leads to $c_{p} / c_{v} \approx 1$ and thus to a vanishing prefactor for the Rayleigh line. The difference between the completely elastic system and the present one lies in the ergodicity of the latter and the non-ergodicity of the former. While a truly elastic one-dimensional system never loses memory of its initial conditions and never completely visits its phase space, the stochastic nature of the collisions quickly eliminates memory of the past and allows for an unhindered exploration of phase space. Thus the assumption of molecular chaos which lies at the heart of hydrodynamic theories is justified for the stochastic system. Therefore it comes as no surprise that a hydrodynamic description yields very accurate results. Similar arguments have been brought forward by Sela and Goldhirsch for the hydrodynamics of a one-dimensional system of particles with a fixed coefficient of restitution [SG.95]: They also find that hydrodynamics apply for their system, but with the difference that an additional hydrodynamic field had to be introduced.

Fig. 3.12 shows the linear relationship between the peak position $\omega_{\max }$ of the Brillouin line and the wave vector $k$. The slope of this line is the adiabatic sound velocity $c_{s}$. The agreement with the theoretical result (the sound velocity of an ideal gas with $c_{p} / c_{v}=1$ is $c_{s}=\sqrt{1 / 2}$ in dimensionless units) is very good. The width of 


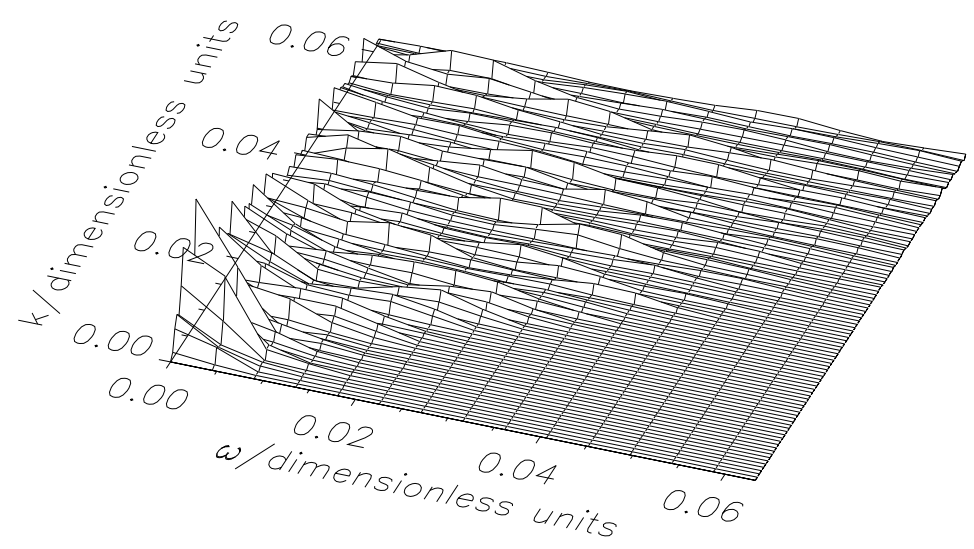

(a)

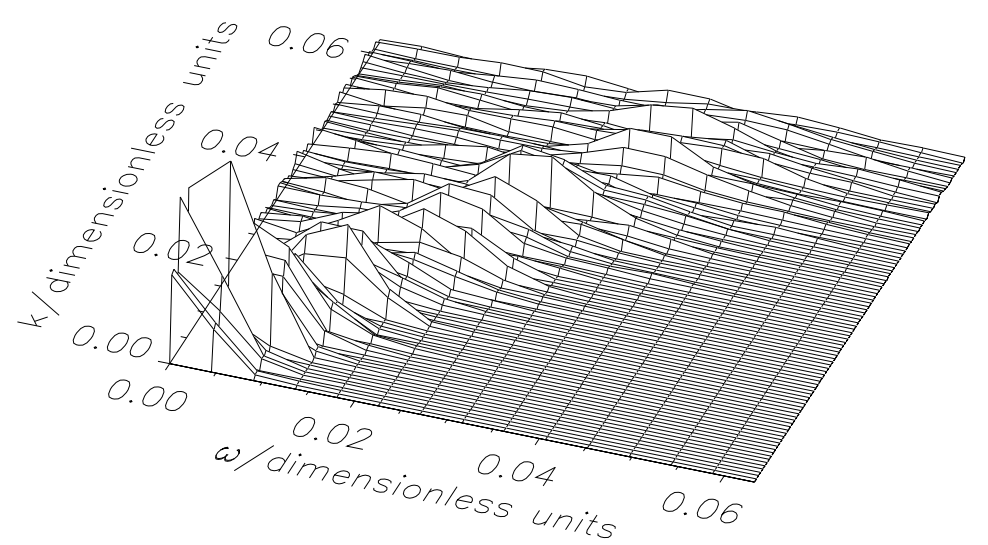

(b)

Figure 3.11.: Dynamic structure factor of a purely elastic system (a) and a system using $p_{\beta}(\epsilon)(\mathrm{b})$ drawn to the same scale. Note the pronounced Brillouin line in (b), indicating soundwave propagation which is also evident in Fig. 3.10 . 


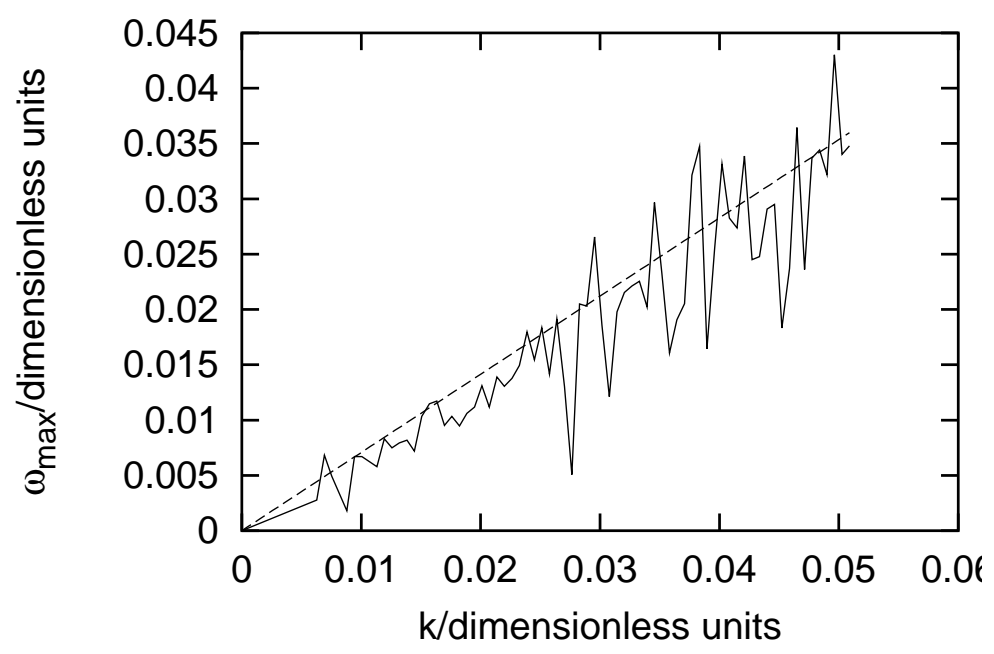

Figure 3.12.: The peak position $\omega_{\max }$ of the Brillouin line from Fig. 3.11 as a function of wave vector $k$. The dashed line is the expected result, corresponding to a sound velocity of $c_{s}=\sqrt{1 / 2}$ in dimensionless units.

the Brillouin line should be proportional to $k^{2}$ [HM86]; the data (not shown here) is consistent with this result although there is too much scatter to draw a decisive conclusion.

The slow approach of the undamped system to equilibrium, as it is shown in Fig. 3.9, is partly due to the fact that thermal damping of the sound mode is small for systems with $c_{p} / c_{v} \approx 1$ [HM86]. More importantly, however, the decay time of a density fluctuation is inversely proportional to its wavevector squared, $k^{-2}$. Therefore the fluctuations with the longest wavelengths survive longest, and this is exactly what can be seen in Fig. 3.9.

Additionally, the static density fluctuations in the system with internal modes are higher than in the ideal gas. This is not shown here but can be inferred from Fig. 3.11(b): The static structure factor $S(k, t=0)$ is proportional to the area under the curve $S(k, \omega)$ for fixed $k$ (by an inverse fourier transformation at $t=0$ ). For an ideal gas, $S(k, t=0)=1$, which is obviously violated in Fig. 3.11(b). This is qualitatively understandable if one keeps in mind that in the system with stochastic coefficient of restitution velocity fluctuations are coupled to density fluctuations by the following mechanism: In a region where particles happen to collide with a higher than average velocity, the translational energy will temporarily be stored in the vibrations, resulting in a mean coefficient of restitution $<1$. This in turn leads to an increased density in that region due to the usual clustering mechanism in granular systems. Analogously, the case of lower than average collision velocity leads to a decreased density. Both effects together result in increased density fluctuations. 


\subsubsection{Damped system}

The next application of the probability distribution constructed in the previous chapter is a long time simulation of 1000 particles with damping, i.e. a simulation where exponential decay of the energy stored in the vibrations is included. This is used to model net dissipation of energy (in contrast to the original model which has overall energy conservation). Simulations of this extended model tend to last very much longer than simulations of undamped systems because situations which would lead to an inelastic collapse in simulations with constant $\epsilon$ take longer to break up if damping is present. They are therefore only accessible with the simulation algorithm using $p_{\beta}(\epsilon)$. The results of this simulation should be comparable to other one-dimensional simulations of granular systems. However, due to the properties of the present model, there is no inelastic collapse which allows for long runs.

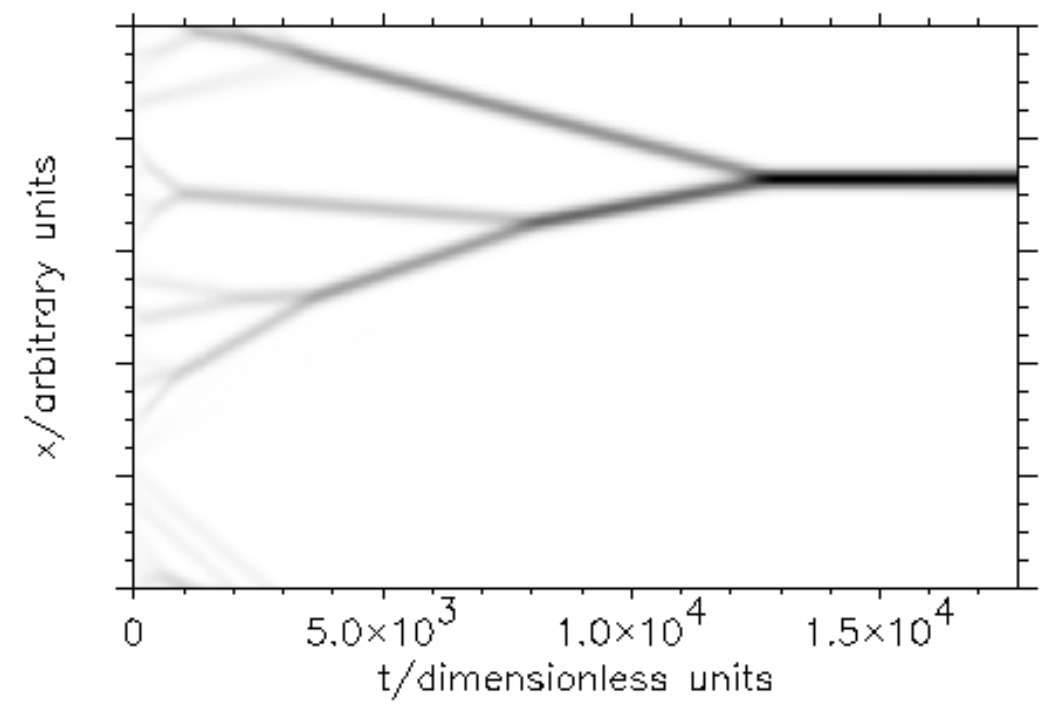

Figure 3.13.: Time evolution of the coarse grained spatial density for a system of 1000 particles with damping. Clusters form quickly and interact completely inelastically when they collide until there is only one cluster left.

Figs. 3.13 and 3.14 show some of the results of this simulation. Not surprisingly, the time evolution of the particle density in Fig. 3.13 shows a clear tendency for clustering. In one dimension, the shape of clusters is naturally considerably simpler than in two or three dimensions (cf. e.g. simulation results in [MY96, G/9.3]). It is seen that the behaviour on a large scale is dominated by relatively simple cluster dynamics. The clusters form very quickly, then drift, collide, and stick until only one big cluster is left. 


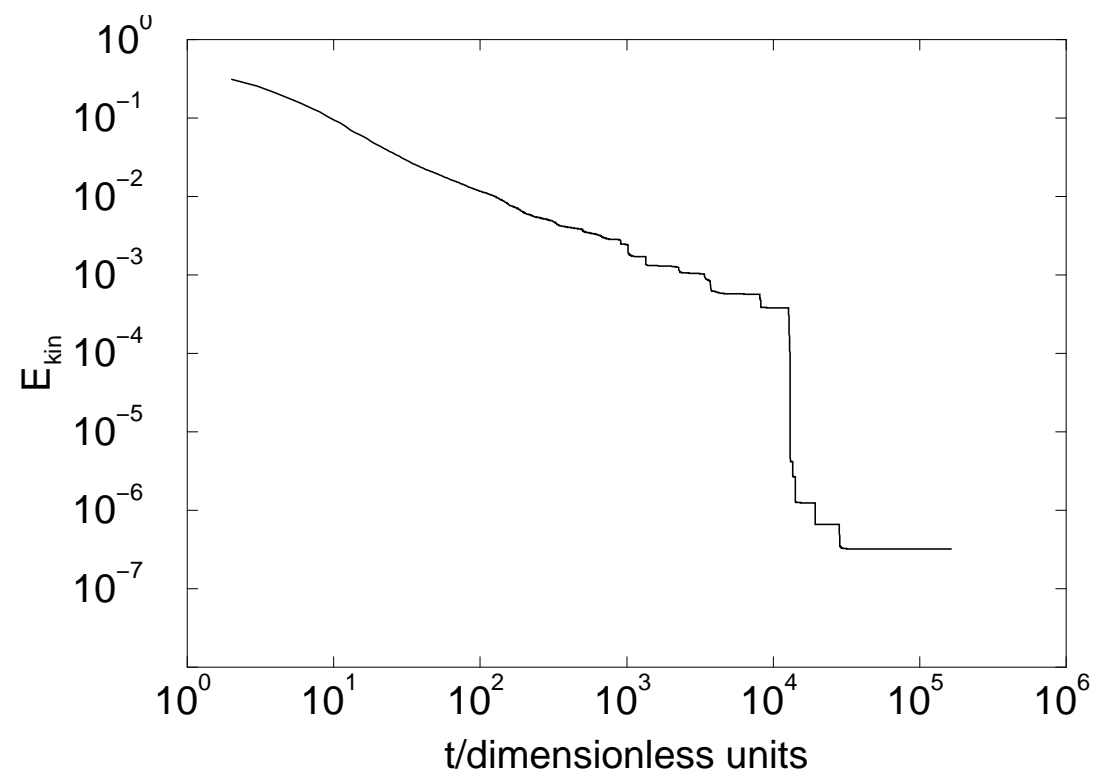

Figure 3.14.: Decay of the translational energy of the system of 1000 particles with damping. The last big step in the curve corresponds to the collision of the last two clusters. By this collision, basically all of the macroscopic kinetic energy is removed and only small "thermal" fluctuations remain.

The energy decay, Fig. 3.14, shows a succession of steps, each of which corresponds to a cluster collision. During each completely inelastic cluster collision a substantial amount of kinetic energy stored in the movement of the clusters is removed from the system. This goes on until the last two clusters collide which removes basically all of the remaining kinetic energy, visible in the giant step at around $t=10^{4}$. Naturally, this behaviour is quite distinct from the homogeneous cooling law, $E_{\mathrm{tr}} \sim t^{-2}$ [Haf83].

\subsection{Summary}

The results of Ch. 2 were used to carry out many particle simulations of particles with internal degrees of freedom. These simulations yielded several noteworthy points:

- The internal degrees of freedom can store energy which is released in situations that would otherwise lead to an inelastic collapse, thus breaking up the collapsing cluster. Hence the model effectively avoids the inelastic collapse, with or without damping.

- The particles undergo complex cluster dynamics including an interplay of formation, drift, collisions, and breakup of clusters on their way from their homogeneous initial state to the equipartitioned equilibrium state, when no damping 
is present. With damping, the dynamics are less complicated since clusters only form, drift, and stick on collisions, but do not break up any more. The final state is one big cluster in this case.

- The undamped equilibrium system behaves exactly as predicted by hydrodynamic theories which is unusual for a one-dimensional system. It is explained by the fact that the stochastic nature of collisions destroys memory and asserts the molecular chaos assumption.

- The hydrodynamic behaviour of the equilibrium system provided an explanation for the extremely slow approach to equilibrium that could be observed in the nonequilibrium simulations: Decay times of density fluctuations are proportional to their wavelengths squared, thus the longest wavelengths survive for very long times.

Thus the model of internal, vibrational degrees of freedom in granular particles gives rise to a number of interesting many particle effects, not present e.g. in models with constant $\epsilon$. These effects motivated much of the work in this chapter. 


\section{From one to three dimensions}

The previous chapters were concerned with one-dimensional particles and a way to model the uptake of kinetic energy into vibrational energy. The ultimate aim of this work is to generalise this idea to two- and three-dimensional particles since this is more closely related to actual experiments of granular materials or colliding macroscopic bodies in general.

The concept that was introduced in the previous chapters, namely elastically vibrating bodies that interact through a repulsive hard-core potential, can of course be generalised to two or three dimensions. However, the equations of motion that can be written down for even a smooth potential (see below for some of them) are much too difficult to be solved analytically. Thus the hard-core limit cannot be taken to obtain a result equivalent to Eq. (2.18). Moreover, even numerical simulations using a smooth potential are very difficult and time consuming (see [G/99] for an example in two dimensions) since the potential must act between every point on the surface of the first body and every point on the surface of the other body. This would require an extensive numerical integration at every time step. While finite-element methods might be feasible, they would amount to pure phenomenology only, without giving insight into the physical details of the process.

Therefore I develop in this chapter a more general variant of the method presented in Sec. 2.1, first only for one dimension but since this new method is easily generalisable to two or three dimensions, I will proceed to do so. This method then opens up the way to relatively efficient simulations of the complete collision process of two (in principle arbitrarily shaped) particles.

\subsection{One-dimensional elastic model}

Consider again two elastic one-dimensional particles of lengths $l_{1}$ and $l_{2}$ travelling along a line and colliding end-to-end, interacting through the repulsive potential $V_{\alpha}(x)=B e^{-\alpha r}$ between the endpoints (separated by a distance $r$ ) as in Sec. 2.1. Once again, the Hamilton function is given by Eq. (2.1). However, while in Sec. 2.1.1 the continuum limit of infinitely many modes $\left(N_{\text {mod }} \rightarrow \infty\right)$ was carried out before the hard-core limit was performed, it is also of interest to see what happens if there are indeed only a finite number of modes. This is e.g. the case when the particles 
are considered to be composed of a number of "atoms" connected by springs. The special case of two atoms connected by a spring has been treated in [BBW63] but here I am interested in the general case.

\subsection{Hard-core solution of the equations of motion}

In Sec. 2.1.1 the derivation of the equations of motion was already given. The result was the nonlinear integral equation (2.12). Here, I will follow the path that was deferred back there, which is to calculate the solution of Eq. (2.12) in the hard core limit without assuming an infinite number of modes. However, since the details of the solution are rather technical and do not illuminate the physical understanding, they are delegated to App. B. Here, I only cite the result (the notation of this chapter is the same as that of Sec. 2.1):

$$
\ddot{R}(t)=-\sum_{\left\{t_{n}\right\}} \frac{2 \dot{r}\left(t_{n}^{-}\right)}{\gamma} \delta\left(t-t_{n}\right)
$$

where the sum runs over the discrete set of times $\left\{t_{n}\right\}$ where the endpoints touch, and $\dot{r}\left(t_{n}^{-}\right)$is the relative velocity of the endpoints at these times (or rather immediately before). The constant $\gamma=\tilde{G}^{\prime}\left(0^{+}\right)$is the initial slope of $\tilde{G}$. The excitation of the modes can then easily be calculated from Eq. (2.7). Note, however, that the times $t_{n}$ can not be calculated beforehand, but have to be calculated "along the way": At first only $t_{1}$ may be calculated (either analytically, but more likely numerically), allowing to compute the changes in velocity and excitation of the modes at that time. After that, one can calculate $t_{2}$ and so on.

Each of the $\delta$-collisions results in a reversal of the relative velocity of the endpoints, as can be verified by plugging Eq. (4.1) into the expression for the end-to-end distance (taken from the exponent in Eq. (2.12))

$$
\begin{aligned}
r(t) & =R\left(T_{0}\right)+\left(t-T_{0}\right) \dot{R}\left(T_{0}\right)+(\tilde{G} * \ddot{R})(t)+Q(t) \\
& =R\left(T_{0}\right)+\left(t-T_{0}\right) \dot{R}\left(T_{0}\right)+\int_{T_{0}}^{t} \tilde{G}\left(t-t^{\prime}\right) \ddot{R}\left(t^{\prime}\right) d t+Q(t) \\
& =R\left(T_{0}\right)+\left(t-T_{0}\right) \dot{R}\left(T_{0}\right)-\sum_{\left\{t_{n}\right\}} \frac{2 \dot{r}\left(t_{n}^{-}\right)}{\gamma} \tilde{G}\left(t-t_{n}\right)+Q(t)
\end{aligned}
$$

and taking the derivative,

$$
\dot{r}(t)=\dot{R}\left(T_{0}\right)-\sum_{\left\{t_{n}\right\}} \frac{2 \dot{r}\left(t_{n}^{-}\right)}{\gamma} \tilde{G}^{\prime}\left(t-t_{n}\right)+Q^{\prime}(t) .
$$


The end-to-end velocity $\dot{r}(t)$ immediately before a particular $\delta$-collision at time $t_{m}$ is thus given by (remember $\tilde{G}^{\prime}\left(0^{-}\right)=0, \tilde{G}^{\prime}\left(0^{+}\right)=\gamma$ )

$$
\dot{r}\left(t_{m}^{-}\right)=\dot{R}\left(T_{0}\right)-\sum_{\left\{t_{n} \mid t_{n}<t_{m}\right\}} \frac{2 \dot{r}\left(t_{n}^{-}\right)}{\gamma} \tilde{G}^{\prime}\left(t-t_{n}\right)+Q^{\prime}(t),
$$

while immediately after the collision it is

$$
\begin{aligned}
\dot{r}\left(t_{m}^{+}\right) & =\dot{R}\left(T_{0}\right)-\sum_{\left\{t_{n} \mid t_{n}<t_{m}\right\}} \frac{2 \dot{r}\left(t_{n}^{-}\right)}{\gamma} \tilde{G}^{\prime}\left(t-t_{n}\right)-2 \dot{r}\left(t_{m}^{-}\right)+Q^{\prime}(t) \\
& =-\dot{r}\left(t_{m}^{-}\right) .
\end{aligned}
$$

This velocity reversal seems very natural and could even have been guessed from the start: In a very short time interval around the $\delta$-collision, the "atoms" at the endpoints only feel their hard core interaction and do not notice the springs they are connected to, so they simply bounce off each other as in a simple elastic hard-core collision.

The difference between the approach of Ch. 2.1 and the present approach can be summarised as follows: The solution of Ch. 2.1 relies on the choice of a particular infinite set of vibrational modes (namely the modes of homogeneous rods). It is thus not completely general, but it results in an elegant equation of motion containing memory terms, Eq. (2.18). Additionally, the collision processes which are described by that equation allow for lasting contacts between the endpoints, see Sec. 2.1.2. On the other hand, the method shown in this chapter is very general as long as only a finite set of modes is used, but there is no closed expression which describes the complete collision process because the times $t_{n}$ need to be determined somehow. A further difference is that there can be no lasting contacts since only $\delta$-collisions occur.

Interestingly, it has so far not been possible to reobtain the result Eq. (2.18) by performing the limit $N_{i}^{\text {mod }} \rightarrow \infty$ from Eq. (4.1). Nevertheless it is the belief of the author that the order of the limits $N_{i}^{\text {mod }} \rightarrow \infty$ and $\alpha \rightarrow \infty$ is unimportant and that only mathematical difficulty prevents the proof. Partly responsible for the failure is the fact that this is a very singular limit: $\tilde{G}(t)$ becomes singular, the $\delta$-functions in Eq. (4.1) are concentrated at points which become dense during what is going to become a lasting contact, the end-to-end distance $r$ goes to zero but not necessarily its derivatives, and so on.

While this result of multiple $\delta$-collisions is not new [BBW63, Wid58], to the best knowledge of the author this is the first time that it has been considered in complete generality for an arbitrary number of modes and has been derived by explicitly performing the hard-core limit of some equations of motion. 


\subsection{Energy considerations}

Having seen that a complete collision process consists of a number of $\delta$-collisions, I will show now that the change in the relative velocity of the centres of mass during such an event can also be calculated by a simple energy balance. The change in relative velocity is denoted by $C$ such that

$$
\begin{aligned}
\ddot{R} & =C \delta\left(t-t_{\mathrm{c}}\right) \\
\dot{R} & =\dot{R}\left(t_{c}^{-}\right)+C \theta\left(t-t_{c}\right)
\end{aligned}
$$

for $t$ in an interval around $t_{\mathrm{c}}$ small enough such that it contains only the one collision event at $t=t_{\mathrm{c}}$. The symbols $t_{c}^{-}$and $t_{c}^{+}$are used to denote the times immediately before and after the collision. The two contributions to the total-energy difference before and after the collision, namely the translational and the vibrational part, will now be computed.

The translational part is given by

$$
\Delta E_{\text {trans }}=\frac{\mu}{2}\left(\left(\dot{R}\left(t_{c}^{-}\right)+C\right)^{2}-\dot{R}^{2}\left(t_{c}^{-}\right)\right)=\mu \dot{R}\left(t_{c}^{-}\right) C+\frac{\mu}{2} C^{2} .
$$

The vibrational energy before and after the collision can be written as in Eq. (2.1)

$$
E_{\mathrm{vib}}^{ \pm}=\sum_{i=1}^{2} \sum_{\nu=1}^{N_{i}^{\bmod }}\left(\frac{p_{i \nu}^{2}\left(t_{c}^{ \pm}\right)}{2 m_{i}}+\frac{m_{i} \omega_{i \nu}^{2} q_{i \nu}^{2}\left(t_{c}^{ \pm}\right)}{2}\right)
$$

From Eq. (2.7) one can read off that after the collision, i.e. for $t>t_{c}$,

$$
\begin{array}{r}
q_{i \nu}(t)=q_{i \nu}\left(t_{c}^{-}\right) \cos \left(\omega_{i \nu}\left(t-t_{c}\right)\right)+\frac{p_{i \nu}\left(t_{c}^{-}\right)}{m_{i} \omega_{i \nu}} \sin \left(\omega_{i \nu}\left(t-t_{c}\right)\right)+ \\
C u_{i \nu}^{0} \frac{\mu}{m_{i} \omega_{i \nu}} \sin \left(\omega_{i \nu}\left(t-t_{c}\right)\right),
\end{array}
$$

which means that immediately after the collision, $q_{i \nu}$ has not changed and the new $p_{i \nu}$ is given by

$$
p_{i \nu}\left(t_{c}^{+}\right)=p_{i \nu}\left(t_{c}^{-}\right)+C u_{i \nu}^{0} \mu \text {. }
$$

The change in vibrational energy is thus given by

$$
\Delta E_{\mathrm{vib}}=\sum_{i=1}^{2} \sum_{\nu=1}^{N_{i}^{\mathrm{mod}}}\left(\frac{p_{i \nu}\left(t_{c}^{-}\right) C u_{i \nu}^{0} \mu}{m_{i}}+\frac{\left(C u_{i \nu}^{0} \mu\right)^{2}}{2 m_{i}}\right) .
$$


Noting that $(-1)^{i} \sum_{\nu=1}^{N_{i}^{\text {mod }}} \frac{p_{i \nu} u_{i \nu}^{0}}{m_{i}}$ is just the velocity of the endpoint of rod $i$ relative to its centre of mass, which will be called $v_{i}$, this result can also be written as

$$
\Delta E_{\mathrm{vib}}=C \mu\left(v_{2}\left(t_{c}^{-}\right)-v_{1}\left(t_{c}^{-}\right)\right)+C^{2} \frac{\mu^{2}}{2} \underbrace{\sum_{i=1}^{2} \frac{1}{m_{i}} \sum_{\nu=1}^{N_{i}^{\bmod }}\left(u_{i \nu}^{0}\right)^{2}}_{=: D} .
$$

The sums in the last term of this expression are merely a constant, depending only on the masses and normal modes of the two particles. It will be named $D$.

The total energy is conserved, thus the energy difference must vanish:

$$
\Delta E_{\text {trans }}+\Delta E_{\text {vib }}=0 \text {. }
$$

This gives rise to a quadratic equation in $C$ when Eqs. (4.11) and (4.16) are inserted. Its two solutions are

$$
C=0
$$

and

$$
C=-2 \frac{\dot{R}\left(t_{c}^{-}\right)+v_{2}\left(t_{c}^{-}\right)-v_{1}\left(t_{c}^{-}\right)}{1+\mu D} .
$$

The former corresponds to the case of no potential (if the particles simply ignored each other, there would of course be no change in energy), the latter is the relevant solution for the present case. It states that the velocity transfer is determined by the relative velocity of the endpoints just before the collision, but as compared to the case of ridgid bodies, the result is also modified by a factor of $1 /(1+\mu D)$ which reflects the influence of the elastic modes in their ability to reduce momentum transfer. The case of ridgid bodies is included in Eq. (4.19) as a special case: When one uses zero modes for both particles, $D=0$ but also $v_{1}=v_{2}=0$ and one thus recovers the well known simple velocity reversal for the collision of two ridgid bodies in one dimension. Note that in this case there is only one $\delta$-collision whereas for a higher number of modes there will in general be a whole sequence of such collisions.

Comparison of Eqs. (4.19) and (4.1) shows that the energy balance gives indeed rise to the same result that was obtained before (recalling the definition of the Green function $\tilde{G}$ from Sec. 2.1.1 and of the constant $D$ in Eq. (4.16)).

\subsection{Two- and three-dimensional objects}

The results of the preceding sections, namely that (a) a complete collision process consists of a number of $\delta$-collisions and (b) the change of velocity in each of these 
$\delta$-collisions can be derived from an energy balance, can be generalised to higher dimensional objects, i.e. $d=2$ or 3 . As for the one-dimensional case, the exact form of the normal vibrational modes of these objects is not important for the calculation. For later applications, however, it shall be mentioned that the normal modes of e.g. spheres are known [Lam82]. Here, I will only present the results for three-dimensional bodies since two-dimensional ones can be regarded as a special case.

Between the $\delta$-collisions, the particles behave according to the equations of motion of free (but possibly elastically vibrating) particles as given in Sec. 4.5. In particular, the linear and angular momenta remain constant. During such a collision, however, there will be three things happening: a change in velocity, a change in angular momentum, and excitation of the elastic modes.

Barring an "accidental" symmetry", there will always be exactly one point of contact at a time $t_{c}$ of a collision. During one such $\delta$-collision there will be a change of relative velocity $\Delta \dot{\boldsymbol{R}}$ which can be written as

$$
\Delta \dot{\boldsymbol{R}}=C \boldsymbol{n},
$$

where $C$ is an as yet unknown number and $\boldsymbol{n}$ is a unit vector normal to the tangent plane at the point of contact. This is due to the fact that within this microscopic description there is no friction involved, thus forces can only act normal to the surface. The coefficient $C$ will be calculated in the following. It will be seen below that it depends on several quantities such as linear and angular momentum.

There will also be a change in angular momentum in the centre of mass frame of reference of each particle due to linear momentum transfer. This is easily computed as

$$
\Delta \boldsymbol{L}_{i}=(-1)^{i} \boldsymbol{X}_{i} \times \mu \Delta \dot{\boldsymbol{R}}
$$

where $\boldsymbol{X}_{i}$ is the vector from the centre of mass of particle $i$ to the point of contact and $\mu$ is the effective mass.

In order to calculate the excitation of the modes, one has to go back to a finiterange potential and derive equations of motion for the amplitudes of the modes. Compared to the one-dimensional situation, the Hamilton function is more complicated here because of the possible presence of rotations which introduce inertial forces into the system. Suffice it to say here that the conjugate momenta $p_{i \nu}$ have a different form than in the one-dimensional case, namely

$$
p_{i \nu}=m_{i} \dot{q}_{i \nu}+J_{i \nu}
$$

where $J_{i \nu}$ is some function of the coordinates $q_{i \nu}$, angular momenta and the tensors of inertia, and that there exists an additional term $\mathcal{H}_{\text {couple }}$ in the Hamilton function

\footnotetext{
${ }^{1}$ Unfortunately, such an accidental symmetry is present in the case of two initially nonvibrating spheres: At some time after the first contact, there might exist a whole contact ring instead of only one point. It will be shown in the next chapter how to deal with this situation.
} 
which is due to the coupling between rotations and vibrations. The details can be found in Sec. 4.5.

Thus the Hamilton function of the system is given by

$$
\mathcal{H}=\mathcal{H}_{\text {trans }}+\mathcal{H}_{\text {vib }}+\mathcal{H}_{\text {rot }}+\mathcal{H}_{\text {couple }}+V_{\alpha}
$$

where

$$
\begin{aligned}
\mathcal{H}_{\text {trans }} & =\frac{\boldsymbol{P}^{2}}{2 \mu} \\
\mathcal{H}_{\text {vib }} & =\sum_{i=1}^{2} \sum_{\nu=1}^{N_{i}^{\text {mod }}}\left(\frac{p_{i \nu}^{2}}{2 m_{i}}+\frac{m_{i} \omega_{i \nu}^{2} q_{i \nu}^{2}}{2}\right)
\end{aligned}
$$

as before, and

$$
\begin{aligned}
\mathcal{H}_{\text {rot }} & =\frac{1}{2} \sum_{i=1}^{2} \boldsymbol{L}_{i}^{\mathrm{T}} \boldsymbol{I}_{i}^{-1} \boldsymbol{L}_{i} \\
\mathcal{H}_{\text {couple }} & =-\sum_{i=1}^{2} \sum_{\nu=1}^{N_{i}^{\text {mod }}} \frac{J_{i \nu}^{2}}{2 m_{i}} \\
V_{\alpha} & =\alpha B \int_{\text {Surface } 1} d^{d-1} \boldsymbol{x}_{1}^{\prime} \int_{\text {Surface } 2} d^{d-1} \boldsymbol{x}_{2}^{\prime} e^{-\alpha|\boldsymbol{Z}|} .
\end{aligned}
$$

The relative momentum of the centres of mass is given by $\boldsymbol{P}$. The new symbols $\boldsymbol{L}_{i}$ and $\boldsymbol{I}_{i}$ denote the angular momentum and the tensor of inertia of the two (possibly deformed) particles, respectively. The vector $\boldsymbol{Z}$ is directed from the point referenced by $\boldsymbol{x}_{1}^{\prime}$ on the surface of particle 1 to the point referenced by $\boldsymbol{x}_{2}^{\prime}$ on the surface of particle 2. The potential now acts between all pairs of points on different surfaces. The vectors $\boldsymbol{x}_{i}^{\prime}$ (and all other primed vector quantities in the following) are measured in a comoving and corotating frame of reference of particle $i$ (see Fig. 4.1 for illustration). The comoving and corotating frame is a coordinate system whose origin is always located at the centre of mass of a particle, and such that the total angular momentum of the particle (as measured in this frame) is zero at all times. The term "comoving and corotating" will in the following be frequently abbreviated by "comoving" only. It is again assumed that the vibrational normal modes $\boldsymbol{u}_{i \nu}^{\prime}\left(\boldsymbol{x}_{i}^{\prime}\right)$ of the particles and their frequencies $\omega_{i \nu}$ are known.

There are four contributions to the vector $\boldsymbol{Z}$ : the vector $\boldsymbol{R}$ connecting the centres of mass of the bodies, the undisplaced locations $\boldsymbol{x}_{i}^{\prime}$ of the points on the surface, the displacement due to vibrations, and the displacement due to rotations. The rotations that take a vector $\boldsymbol{x}_{i}^{\prime}$ from the comoving coordinate system to the regular inertial 


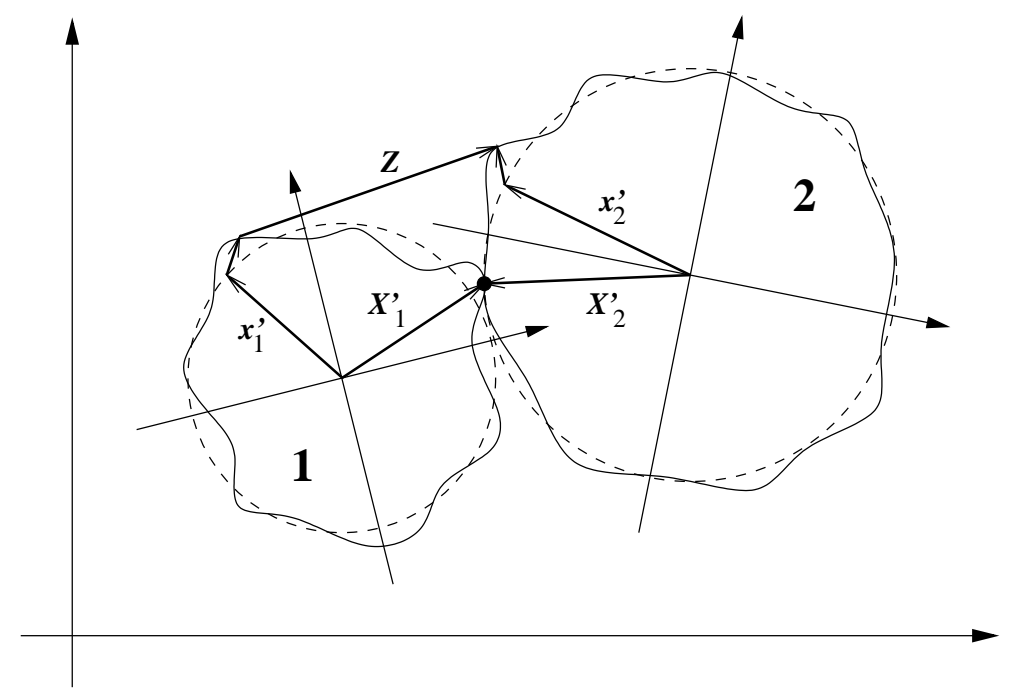

Figure 4.1.: Two spheres and their comoving frames of reference, embedded in an outer inertial coordinate system. The vectors $\boldsymbol{x}_{i}^{\prime}$ point from the centre of mass of sphere $i$ to an arbitrary point on the undeformed surface. From there, two displacement vectors point to the corresponding points on the deformed surfaces. The vector $\boldsymbol{Z}$ connects these two points. The vectors $\boldsymbol{X}_{i}^{\prime}$ point to the point of contact.

system will be denoted by rotation matrices $\boldsymbol{T}_{i}$. Thus $\boldsymbol{Z}$ is given by (see also Fig. 4.1)

$$
\boldsymbol{Z}=\boldsymbol{R}+\boldsymbol{T}_{2}\left(\boldsymbol{x}_{2}^{\prime}+\sum_{\nu} q_{2 \nu} \boldsymbol{u}_{2 \nu}^{\prime}\left(\boldsymbol{x}_{2}^{\prime}\right)\right)-\boldsymbol{T}_{1}\left(\boldsymbol{x}_{1}^{\prime}+\sum_{\nu} q_{1 \nu} \boldsymbol{u}_{1 \nu}^{\prime}\left(\boldsymbol{x}_{1}^{\prime}\right)\right)
$$

As in the one dimensional case, the equations of motion for $\boldsymbol{R}$ and $q_{i \nu}$ can now be written down:

$$
\begin{aligned}
\mu \ddot{\boldsymbol{R}}= & -\alpha^{2} B \int d^{d-1} \boldsymbol{x}_{1}^{\prime} d^{d-1} \boldsymbol{x}_{2}^{\prime} \frac{\boldsymbol{Z}}{|\boldsymbol{Z}|} e^{-\alpha|\boldsymbol{Z}|}, \\
\dot{p}_{i \nu}= & m_{i} \ddot{q}_{i \nu}+\dot{J}_{i \nu} \\
= & -m_{i} \omega_{i \nu}^{2} q_{i \nu}-\alpha^{2} B \int d^{d-1} \boldsymbol{x}_{1}^{\prime} d^{d-1} \boldsymbol{x}_{2}^{\prime} \frac{\boldsymbol{Z}^{\mathrm{T}}}{|\boldsymbol{Z}|} e^{-\alpha|\boldsymbol{Z}|} \boldsymbol{T}_{i} \boldsymbol{u}_{i \nu}^{\prime}\left(\boldsymbol{x}_{i}^{\prime}\right)(-1)^{i}- \\
& \frac{1}{2} \boldsymbol{L}_{i}^{\mathrm{T}} \frac{\partial \boldsymbol{I}_{i}^{-1}}{\partial q_{i \nu}} \boldsymbol{L}_{i}+\sum_{\mu=1}^{N_{i}^{\bmod }} \frac{J_{i \mu}}{m_{i}} \frac{\partial J_{i \nu}}{\partial q_{i \nu}} .
\end{aligned}
$$

Since the potential $V_{\alpha}$ is sharply peaked for large $\alpha$, one can replace $\boldsymbol{x}_{i}^{\prime}$ by $\boldsymbol{X}_{i}^{\prime}$, the vector from the centre of mass to the point of contact, in the the term $\boldsymbol{u}_{i \nu}^{\prime}\left(\boldsymbol{x}_{i}^{\prime}\right)$ in the integral in Eq. (4.31) without introducing errors when letting $\alpha \rightarrow \infty$. The first 
part of the integral is then identical to the one in Eq. (4.30) which means that one can insert it into Eq. (4.31) to give

$$
\ddot{q}_{i \nu}=-\omega_{i \nu}^{2} q_{i \nu}+\frac{\mu}{m_{i}} \ddot{\boldsymbol{R}}^{\mathrm{T}} \boldsymbol{T}_{i} \boldsymbol{u}_{i \nu}^{\prime}\left(\boldsymbol{X}_{i}^{\prime}\right)(-1)^{i}+\mathcal{O}(\boldsymbol{L})
$$

to zeroth order in angular momentum. One could also go to the next higher order since the equations retain their harmonic-oscillator structure in that case. This would, however, only complicate the matter without giving additional physical insight. To zeroth order, the conjugate momenta are given by their familiar nonrotating value, i.e. $p_{i \nu}=m q_{i \nu}$. Refer to Sec. 4.5 for details of the approximation of the equations of motion to zeroth and the next higher order.

Eq. (4.32) is formally equivalent to Eq. (2.5). This means that one can proceed in a similar manner as in the one-dimensional case. Following the same arguments as before, the change in momentum of a mode after a collision is now given by

$$
p_{i \nu}\left(t_{c}^{+}\right)=p_{i \nu}\left(t_{c}^{-}\right)+C \mu \boldsymbol{n}^{\mathrm{T}} \boldsymbol{T}_{i} \boldsymbol{u}_{i \nu}^{\prime}\left(\boldsymbol{X}_{i}^{\prime}\right)(-1)^{i} .
$$

Eqs. (4.20), (4.21) and (4.33) provide enough information to write down the energy balance,

$$
\Delta E_{\mathrm{trans}}+\Delta E_{\mathrm{rot}}+\Delta E_{\mathrm{vib}}=0
$$

The energies turn out to be, after a little algebra,

$$
\begin{aligned}
\Delta E_{\mathrm{trans}} & =C \mu \dot{\boldsymbol{R}}^{\mathrm{T}} \boldsymbol{n}+C^{2} \frac{\mu}{2} \\
\Delta E_{\mathrm{rot}} & =\sum_{i=1}^{2}\left((-1)^{i} C \mu \boldsymbol{L}_{i}^{\mathrm{T}} \boldsymbol{I}_{i}^{-1}\left(\boldsymbol{X}_{i} \times \boldsymbol{n}\right)+\frac{\mu^{2} C^{2}}{2}\left(\boldsymbol{X}_{i} \times \boldsymbol{n}\right)^{\mathrm{T}} \boldsymbol{I}_{i}^{-1}\left(\boldsymbol{X}_{i} \times \boldsymbol{n}\right)\right) \\
\Delta E_{\mathrm{vib}} & =C \mu \boldsymbol{n}^{\mathrm{T}} \sum_{i=1}^{2}(-1)^{i} \underbrace{\sum_{\nu=1}^{N_{i}^{\mathrm{mod}}} \frac{p_{i \nu}\left(t_{c}^{-}\right) \boldsymbol{T}_{i} \boldsymbol{u}_{i \nu}^{\prime}\left(\boldsymbol{X}_{i}^{\prime}\right)}{m_{i}}}_{=: \boldsymbol{v}_{i}\left(t_{c}^{-}\right)}+\frac{C^{2} \mu^{2}}{2} \underbrace{\sum_{i=1}^{2} \sum_{\nu=1}^{N_{i}^{\bmod }} \frac{\left(\boldsymbol{n}^{\mathrm{T}} \boldsymbol{T}_{i} \boldsymbol{u}_{i \nu}^{\prime}\left(\boldsymbol{X}_{i}^{\prime}\right)\right)^{2}}{m_{i}} .}_{=: D} .
\end{aligned}
$$

The abbreviation $\boldsymbol{v}_{i}\left(t_{c}^{-}\right)$in the first term of the expression for $\Delta E_{\mathrm{vib}}$ denotes the vibrational part of the velocity of the point of contact of particle $i$ before the collision.

This gives, as before, a quadratic equation in $C$. Apart from the trivial solution $C=0$, it is solved by

$$
C=-2 \frac{\dot{\boldsymbol{R}}+\boldsymbol{v}_{2}-\boldsymbol{v}_{1}+\boldsymbol{\omega}_{2} \times \boldsymbol{X}_{2}-\boldsymbol{\omega}_{1} \times \boldsymbol{X}_{1}}{1+\mu \sum_{i=1}^{2}\left(\boldsymbol{X}_{i} \times \boldsymbol{n}\right)^{\mathrm{T}} \boldsymbol{I}_{i}^{-1}\left(\boldsymbol{X}_{i} \times \boldsymbol{n}\right)+\mu D} \boldsymbol{n}
$$


where $\boldsymbol{\omega}_{i}=\boldsymbol{I}_{i}^{-1} \boldsymbol{L}_{i}\left(t_{c}^{-}\right)$is the angular velocity of particle $i$ at time $t_{c}^{-}$. This expression for $C$ can now be used in Eqs. (4.20) and (4.21).

This result has again a fairly simple interpretation: The important quantity is the relative velocity of the points of contact immediately before the time of contact, which appears in the numerator of Eq. (4.38). It consists of three contributions, the motion of the centres of mass, the vibrations, and the rotations. The denominator describes to which extent the momentum transfer is reduced by the rotations (the middle term) and vibrations (the term $\mu D$ ). The quantity $D$ is here no longer a material constant as in the one-dimensional case but depends on the particular vibrational and rotational state through $\boldsymbol{n}, \boldsymbol{T}_{i}$ and $\boldsymbol{X}_{i}^{\prime}$.

Again, the case of ridgid hard spheres is included in Eq. (4.38): When one specialises to zero modes, $D=\boldsymbol{v}_{i}=0$ and all the terms containing $\boldsymbol{X}_{i}$ vanish since $\boldsymbol{X}_{i}$ is always parallel to $\boldsymbol{n}$ for spheres. What remains is simply a reversal of the velocity component normal to the surface at the point of contact, as should be the case for hard spheres.

\subsection{Differential equations of the free motion of an elastically vibrating body}

Inbetween two $\delta$-collisions, the two bodies do not interact and behave according to the laws of classical mechanics. In this section, the equations of motion for such a freely flying, elastically vibrating, rotating body will be derived.

The equations of motion for a freely flying, rigid body are the well known Euler equations (see e.g. [LL.91b] or any other standard textbook on classical mechanics). Here, they are of little use since the tensor of inertia of a vibrating body is in general not constant in time, even its system of principal axes is not. Additionally, inertial forces appear due to rotations which influence the vibrations. While the Euler equations are equations for the angular velocity only, it is furthermore required here to calculate the full rotation matrix $\boldsymbol{T}=\exp (\phi \boldsymbol{J})$ that rotates the body from its initial position into its present state. Here $\boldsymbol{J}$ is the vector of infinitesimal generators (cf. e.g. [Sch.90, p. 90]),

$$
\boldsymbol{J}_{1}=\left(\begin{array}{ccc}
0 & 0 & 0 \\
0 & 0 & -1 \\
0 & 1 & 0
\end{array}\right), \quad \boldsymbol{J}_{2}=\left(\begin{array}{ccc}
0 & 0 & 1 \\
0 & 0 & 0 \\
-1 & 0 & 0
\end{array}\right), \quad \boldsymbol{J}_{3}=\left(\begin{array}{ccc}
0 & -1 & 0 \\
1 & 0 & 0 \\
0 & 0 & 0
\end{array}\right)
$$

and $\phi$ is the vector whose direction specifies the axis of rotation and whose length is given by the angle of rotation. In the following, a derivation of the equations of motion for $\phi$ and the excitations of the modes is given.

In this section, the meaning of the symbols $\rho, q_{\nu}, \omega_{\nu}, \boldsymbol{L}, \boldsymbol{T}, \boldsymbol{I}$, and $\boldsymbol{u}_{\nu}^{\prime}$ will be the same as in Sec. 4.4 but without particle index for notational ease. 


\subsubsection{Equations of motion for the rotation}

Since angular momentum is conserved, $\boldsymbol{L}=$ const., the equations of motion for the rotation are simple to derive. The rotation matrix $\boldsymbol{T}=\exp (\boldsymbol{\phi} \boldsymbol{J})$ can be written explicitly as

$$
\boldsymbol{T}=\mathbf{1} \cos (\phi)+\hat{\boldsymbol{\phi}} \hat{\boldsymbol{\phi}}^{\mathrm{T}}(1-\cos (\phi))+(\hat{\boldsymbol{\phi}} \boldsymbol{J}) \sin (\phi)
$$

with $\phi=|\phi|, \hat{\phi}=\phi / \phi$. The angular velocity $\boldsymbol{\omega}$ is connected to $\dot{\phi}$ through

$$
\omega=A \dot{\phi}
$$

where the matrix $\boldsymbol{A}$ is given by

$$
\boldsymbol{A}=1 \frac{\sin (\phi)}{\phi}+\hat{\boldsymbol{\phi}} \hat{\boldsymbol{\phi}}^{\mathrm{T}}\left(1-\frac{\sin (\phi)}{\phi}\right)+(\hat{\boldsymbol{\phi}} \boldsymbol{J}) \frac{1-\cos (\phi)}{\phi} .
$$

While this doesn't seem to be standard textbook lore, it can be checked by a (tedious) calculation. The basic idea to derive this result is to notice that the velocity of a vector $\boldsymbol{x}$ which is rotated by a time-dependent rotation matrix $\boldsymbol{T}$ is on the one hand given by $\dot{\boldsymbol{T}} \boldsymbol{x}$ and on the other hand in terms of the angular velocity by $\boldsymbol{\omega} \times(\boldsymbol{T} \boldsymbol{x})=$ $(\boldsymbol{\omega} \boldsymbol{J}) \boldsymbol{T} \boldsymbol{x}$. Since this holds for arbitrary $\boldsymbol{x}$, it follows that

$$
\dot{\boldsymbol{T}}=\sum_{j=1}^{3} \dot{\phi}_{j} \frac{\partial \boldsymbol{T}}{\partial \phi_{j}}=\left(\sum_{j=1}^{3} \omega_{j} \boldsymbol{J}_{j}\right) \boldsymbol{T}
$$

This asserts the linear relationship between $\boldsymbol{\omega}$ and $\dot{\boldsymbol{\phi}}$. The precise form of the corresponding matrix $\boldsymbol{A}$ that connects $\boldsymbol{\omega}$ and $\dot{\boldsymbol{\phi}}$, Eq. (4.42), can be obtained by solving Eq. (4.43) for $\boldsymbol{\omega}$.

Now the necessary ingredients are available to write down the equation for the rotation matrix $\boldsymbol{T}$ or rather the rotation vector $\boldsymbol{\phi}(\boldsymbol{I}$ is the time-dependent tensor of inertia, $\boldsymbol{I}^{\prime}$ is the same but in the comoving frame):

$$
\begin{aligned}
\boldsymbol{L} & =\boldsymbol{I} \boldsymbol{\omega}=\boldsymbol{T} \boldsymbol{I}^{\prime} \boldsymbol{T}^{\mathrm{T}} \boldsymbol{A} \dot{\boldsymbol{\phi}} \\
\text { thus } & \dot{\boldsymbol{\phi}}=\boldsymbol{A}^{-1} \boldsymbol{T} \boldsymbol{I}^{-1} \boldsymbol{T}^{\mathrm{T}} \boldsymbol{L} .
\end{aligned}
$$

The inverse of $\boldsymbol{A}$ can be calculated explicitly:

$$
\boldsymbol{A}^{-1}=\hat{\boldsymbol{\phi}} \hat{\boldsymbol{\phi}}^{\mathrm{T}}+\left(\mathbf{1}-\hat{\boldsymbol{\phi}} \hat{\boldsymbol{\phi}}^{\mathrm{T}}\right) \frac{\phi \sin (\phi)}{2-2 \cos (\phi)}-(\hat{\boldsymbol{\phi}} \boldsymbol{J}) \frac{\phi}{2} .
$$

Eq. (4.45) is a highly nonlinear differential equation for $\phi$ which is explicitly time dependent through $\boldsymbol{I}^{\prime-1}$. Since $\boldsymbol{L}$ is merely a constant, the only remaining question is how the tensor of inertia changes due to vibrations. This will be given in Sec. 4.5.3. 


\subsubsection{Equations of motion for the modes}

The starting point is the Lagrangian $\mathcal{L}=E_{\text {kin }}-E_{\text {pot }}$. The motion of the centre of mass will be disregarded here since it decouples and has a trivial time evolution. Then the kinetic energy part is given by

$$
\begin{aligned}
E_{\text {kin }}= & \int_{\text {Object }} d^{3} x^{\prime} \frac{\rho}{2}\left(\frac{d}{d t} \boldsymbol{T}\left(\boldsymbol{x}^{\prime}+\sum_{\mu} q_{\mu} \boldsymbol{u}_{\mu}^{\prime}\left(\boldsymbol{x}^{\prime}\right)\right)\right)^{2} \\
= & \int_{\text {Object }} d^{3} x^{\prime} \frac{\rho}{2}\left[\left(\dot{\boldsymbol{T}}\left(\boldsymbol{x}^{\prime}+\sum_{\mu} q_{\mu} \boldsymbol{u}_{\mu}^{\prime}\left(\boldsymbol{x}^{\prime}\right)\right)\right)^{2}+\right. \\
& 2\left(\dot{\boldsymbol{T}}\left(\boldsymbol{x}^{\prime}+\sum_{\mu} q_{\mu} \boldsymbol{u}_{\mu}^{\prime}\left(\boldsymbol{x}^{\prime}\right)\right)\right)^{\mathrm{T}}\left(\boldsymbol{T} \sum_{\mu} \dot{q}_{\mu} \boldsymbol{u}_{\mu}^{\prime}\left(\boldsymbol{x}^{\prime}\right)\right)+ \\
& \left.\left(\boldsymbol{T} \sum_{\mu} \dot{q}_{\mu} \boldsymbol{u}_{\mu}^{\prime}\left(\boldsymbol{x}^{\prime}\right)\right)^{2}\right]
\end{aligned}
$$

The first part of this expression is the purely rotational energy of the deformed body, which can also be expressed as

$$
E_{\mathrm{rot}}=\frac{1}{2} \boldsymbol{L}^{\mathrm{T}} \boldsymbol{I}^{-1} \boldsymbol{L}
$$

The last part is the purely vibrational energy which is, due to the orthonormality of the modes,

$$
E_{\mathrm{vib}}=\sum_{\nu} \frac{m \dot{q}_{\nu}^{2}}{2}
$$

The crossterms, however, do not vanish and give rise to an additional term in the Hamiltonian later on.

The potential energy is

$$
E_{\mathrm{pot}}=\sum_{\nu} \frac{m \omega_{\nu}^{2} q_{\nu}^{2}}{2}
$$

and does not depend on $\dot{q}_{\nu}$. Therefore the conjugate momenta are given by

$$
p_{\nu}=\frac{\partial \mathcal{L}}{\partial \dot{q}_{\nu}}=m \dot{q}_{\nu}+\int_{\text {Object }} d^{3} x^{\prime} \rho\left(\dot{\boldsymbol{T}}\left(\boldsymbol{x}^{\prime}+\sum_{\mu} q_{\mu} \boldsymbol{u}_{\mu}^{\prime}\left(\boldsymbol{x}^{\prime}\right)\right)\right)^{\mathrm{T}} \boldsymbol{T} \boldsymbol{u}_{\nu}^{\prime}\left(\boldsymbol{x}^{\prime}\right)
$$


They differ from the conjugate momenta in one dimension. Using the relation from Eq. (4.43) and the well-known expression for the angular velocity,

$$
\begin{aligned}
\dot{\boldsymbol{T}} & =(\boldsymbol{\omega} \boldsymbol{J}) \boldsymbol{T} \text { and } \\
\boldsymbol{\omega} & =\boldsymbol{I}^{-1} \boldsymbol{L},
\end{aligned}
$$

this can also be written as

$$
\begin{aligned}
p_{\nu} & =m \dot{q}_{\nu}+\left(\left(\boldsymbol{I}^{\prime}\right)^{-1} \boldsymbol{T}^{-1} \boldsymbol{L}\right)^{\mathrm{T}} \int d^{3} x^{\prime} \rho\left(\boldsymbol{x}^{\prime}+\sum_{\mu} q_{\mu} \boldsymbol{u}_{\mu}^{\prime}\left(\boldsymbol{x}^{\prime}\right)\right) \times \boldsymbol{u}_{\nu}^{\prime} \\
& =: m \dot{q}_{\nu}+J_{\nu}\left(\left\{q_{\mu}\right\}, \boldsymbol{L}, \boldsymbol{T}, \boldsymbol{I}^{\prime}\right) .
\end{aligned}
$$

For any object there exist three "vibrational" modes which correspond to rotations of the whole body. Since their frequencies are zero, however, they are usually not used and rotations are treated seperately, like e.g. in this work. These modes have the form $\boldsymbol{u}_{\text {rot }}^{\prime}\left(\boldsymbol{x}^{\prime}\right) \propto \boldsymbol{\omega}^{\prime} \times \boldsymbol{x}^{\prime}$ with the rotation axis defined by $\boldsymbol{\omega}^{\prime}$. They are orthogonal to each other and all of the regular modes. By cyclically exchanging the factors of the triple product $\boldsymbol{\omega}^{\prime \mathrm{T}}\left(\boldsymbol{x}^{\prime} \times \boldsymbol{u}_{\nu}^{\prime}\right)$ in the integral defining $J_{\nu}$ in Eq. (4.55) (where $\left.\boldsymbol{\omega}^{\prime}=\left(\boldsymbol{I}^{\prime}\right)^{-1} \boldsymbol{T}^{-1} \boldsymbol{L}\right)$, it can be seen that due to the orthogonality the first term of the integral vanishes. Thus $J_{\nu}$ takes on the simple form

$$
J_{\nu}=\left(\left(\boldsymbol{I}^{\prime}\right)^{-1} \boldsymbol{T}^{-1} \boldsymbol{L}\right)^{\mathrm{T}} \sum_{\mu} q_{\mu} \int d^{3} x^{\prime} \rho \boldsymbol{u}_{\mu}^{\prime} \times \boldsymbol{u}_{\nu}^{\prime} .
$$

Therefore the Hamiltonian is given by

$$
\begin{aligned}
\mathcal{H} & =E_{\text {kin }}+E_{\text {pot }} \\
& =\frac{1}{2} \boldsymbol{L}^{\mathrm{T}} \boldsymbol{I}^{-1} \boldsymbol{L}+\sum_{\mu} \frac{p_{\mu}-J_{\mu}}{m} J_{\mu}+\sum_{\mu} \frac{\left(p_{\mu}-J_{\mu}\right)^{2}}{2 m}+\sum_{\mu} \frac{m \omega_{\mu}^{2} q_{\mu}^{2}}{2} \\
& =\frac{1}{2} \boldsymbol{L}^{\mathrm{T}} \boldsymbol{I}^{-1} \boldsymbol{L}+\sum_{\mu}\left(\frac{p_{\mu}^{2}}{2 m}+\frac{m \omega_{\mu}^{2} q_{\mu}^{2}}{2}\right)-\sum_{\mu} \frac{J_{\mu}^{2}}{2 m}
\end{aligned}
$$

Here the crossterms and the vibrational energy have been written in terms of $p_{\mu}$ and $J_{\mu}$. From the Hamiltonian one gets the equations of motion for the $q_{\nu}$ :

$$
\begin{aligned}
\dot{p}_{\nu} & =m \ddot{q}_{\nu}+\dot{J}_{\nu}=-\frac{\partial \mathcal{H}}{\partial q_{\nu}} \\
& =-m \omega_{\nu}^{2} q_{\nu}-\frac{\partial}{\partial q_{\nu}} \frac{1}{2} \boldsymbol{L}^{\mathrm{T}} \boldsymbol{I}^{-1} \boldsymbol{L}+\frac{\partial}{\partial q_{\nu}} \sum_{\mu} \frac{J_{\mu}^{2}}{2 m}
\end{aligned}
$$


which is equivalent to

$$
\ddot{q}_{\nu}=-\omega_{\nu}^{2} q_{\nu}-\frac{1}{2 m} \boldsymbol{L}^{\mathrm{T}} \frac{\partial \boldsymbol{I}^{-1}}{\partial q_{\nu}} \boldsymbol{L}+\sum_{\mu} \frac{J_{\mu}}{m^{2}} \frac{\partial J_{\mu}}{\partial q_{\nu}}-\frac{\dot{J}_{\nu}}{m} .
$$

The presence of the last two terms is physically clear: They comprise the effects of inertial forces. The last one contains the Coriolis force and the force due to changes in angular velocity while the second to last one contains the centrifugal force. The situation is complicated by the non-constantness of the tensor of inertia which comes into play in all of the last three terms.

Eq. (4.63) is exact but not very useful since it is very complicated, nonlinear, and couples different modes. What's more, the method presented in Sec. 4.4 relies on the fact that the modes behave like a harmonic oscillator, which is obviously not the case in Eq. (4.63). For small excitations and angular momenta, however, it can be expanded. Here, only the lowest nontrivial order will be kept. Eq. (4.57) shows that the term $\sum_{\mu} \frac{J_{\mu}}{m^{2}} \frac{\partial J_{\mu}}{\partial q_{\nu}}$ is of order $\mathcal{O}\left(L^{2} q\right)$. The term $\dot{J}_{\nu}$ contains a part which is also $\mathcal{O}\left(L^{2} q\right)$, one that is $\mathcal{O}\left(L q^{2}\right)$, and one which is only $\mathcal{O}(L q)$. The second term on the rhs of Eq. (4.63) is $\mathcal{O}\left(L^{2}\right)$. Considering $\boldsymbol{L}$ and $q_{\nu}$ to be both small, the latter two are obviously the leading terms; to this order, the equation looks like

$$
\ddot{q}_{\nu}=-\omega_{\nu}^{2} q_{\nu}-\frac{1}{2 m} \boldsymbol{L}^{\mathrm{T}} \frac{\partial \boldsymbol{I}^{-1}}{\partial q_{\nu}} \boldsymbol{L}-\left(\left(\boldsymbol{I}^{\prime 0}\right)^{-1} \boldsymbol{T}^{-1} \boldsymbol{L}\right)^{\mathrm{T}} \sum_{\mu} \dot{q}_{\mu} \int d^{3} x^{\prime} \rho \boldsymbol{u}_{\mu}^{\prime} \times \boldsymbol{u}_{\nu}^{\prime}+\mathcal{O}\left((\cdot)^{3}\right)
$$

Here, $\boldsymbol{I}^{\prime 0}$ is the tensor of inertia of the undeformed object. The sloppy notation of the higher order terms is to indicate that they contain mixed terms of various orders. This differential equation is now linear and could in principle be diagonalised which would lead to small frequency shifts and mode-mixing, but would retain the structure of a set of decoupled harmonic oscillators. Thus one has the choice to only go to zeroth order and neglect all terms $\mathcal{O}(L)$, yielding a simple harmonic oscillator as in the nonrotating case, or to go one order higher and use Eq. (4.64), depending on the initial conditions and whether the higher order calculation is computationally feasible.

\subsubsection{Change of the tensor of inertia due to vibrations}

For completeness, the change of the tensor of inertia due to vibrations shall be given here. It is a necessary ingredient in order to explicitly write down Eqs. (4.45) and (4.64).

Since this entire work uses merely linear elasticity, it only makes sense to go to first order in the excitations $q_{i \nu}$. From the definition of the tensor of inertia one gets 
in the comoving frame

$$
\begin{aligned}
\left(\boldsymbol{I}^{\prime}\right)_{k l} & =\int_{\text {Object }} d^{d} x^{\prime} \rho\left(\left(\boldsymbol{x}^{\prime}+\sum_{\nu} q_{\nu} \boldsymbol{u}_{\nu}^{\prime}\right)^{2} \delta_{k l}-\left(\boldsymbol{x}^{\prime}+\sum_{\nu} q_{\nu} \boldsymbol{u}_{\nu}^{\prime}\right)_{k}\left(\boldsymbol{x}^{\prime}+\sum_{\nu} q_{\nu} \boldsymbol{u}_{\nu}^{\prime}\right)_{l}\right) \\
& =\left(\boldsymbol{I}^{\prime 0}\right)_{k l}+\sum_{\nu} q_{\nu}\left(\sum_{m=1}^{d} \boldsymbol{M}_{\nu m m}^{\prime} \delta_{k l}-\boldsymbol{M}_{\nu k l}^{\prime}\right)+\mathcal{O}\left(q_{\nu}^{2}\right),
\end{aligned}
$$

where

$$
\boldsymbol{M}_{\nu k l}^{\prime}=\int_{\text {Object }} d^{d} x^{\prime} \rho\left(\left(\boldsymbol{x}^{\prime}\right)_{k}\left(\boldsymbol{u}_{\nu}^{\prime}\right)_{l}+\left(\boldsymbol{x}^{\prime}\right)_{l}\left(\boldsymbol{u}_{\nu}^{\prime}\right)_{k}\right)
$$

is a constant2, depending only on the normal modes and the mass density of the objects. It is a tensor of second rank with respect to its last two indices.

It is in fact necessary to know how the inverse of the tensor of inertia, $\left(\boldsymbol{I}^{\prime}\right)^{-1}$, behaves due to vibrations. To first order, this is

$$
\left(\boldsymbol{I}^{\prime}\right)^{-1}=\left(\boldsymbol{I}^{\prime 0}\right)^{-1}-\sum_{\nu} q_{\nu}\left(\boldsymbol{I}^{\prime 0}\right)^{-1}\left(\operatorname{Tr}\left(\boldsymbol{M}_{\nu}^{\prime}\right) \mathbf{1}-\boldsymbol{M}_{\nu}^{\prime}\right)\left(\boldsymbol{I}^{\prime 0}\right)^{-1}+\mathcal{O}\left(q_{\nu}^{2}\right)
$$

This result can directly be applied to Eq. (4.45). Additionally, after translation from the comoving frame into the inertial frame, it can be differentiated with respect to $q_{\nu}$ and inserted into Eq. (4.64), yielding a term independent of the $q_{\nu}$ but time dependent through the rotation matrix $\boldsymbol{T}$.

\subsection{Consequences of the finite set of modes}

One difference of the method presented here to a continuum description is the following: If the colliding bodies approach very slowly, one expects only a small area of contact (in a quasistatic calculation). However, in order to mimick such a small flat area, modes with a fine surface structure are required. For spheres, such modes have high indices $n$ (see Sec. 5.1 for details on the vibrational modes of a sphere). Thus paradoxically, situations with small velocities require more modes and are thus more difficult to compute than situations with high velocities. This fact is expressed by non-Hertzian behaviour in the simulations as will be shown in the next chapter.

\footnotetext{
${ }^{2}$ For homogeneous spheres, it seems that only a few modes have $M_{\nu k l}^{\prime} \neq 0$, namely those with $\nu=(0, m, l)$ and $\nu=(2, m, l)$. See Sec. 5.1 for details of the modes of spheres and their indexing scheme. This result, however, derives from explicit calculation for the first few modes and is not a proof.
} 
This observation can even be be made more precise by the following analysis: If the initial velocity is small enough, two identical, initially nonvibrating spheres will touch at exactly the same point at each $\delta$-collision, which lies on the line connecting the two centres of mass, or in other words at the origin if the frame of reference is properly chosen. The excitation of the modes after the first $\delta$-collision is proportional to the velocity (since no rotations and vibrations are present at that time, cf. also Eq. (4.38)), but if this velocity is so small that the centres of mass of the spheres hardly move until the next $\delta$-collision, the time at which this next contact occurs is unaffected by the degree of excitation. This is perhaps clarified by looking at the motion of the point on the first sphere which is nearest to the other sphere, the supposed only point of contact: After the first contact, the next one will occur when this point comes back to the origin. Since the modes are harmonic, however, this return time is independent of the absolute strength of excitation of the modes, provided they are all excited in the same relative degree. But this is exactly what happens by varying the initial velocity: All modes are excited in the same relative, albeit not absolute degree. Thus one can convince oneself by induction that the same holds for all following contacts as well. Therefore the times at which the spheres touch and the relative degrees of excitation are unaffected by the initial velocity. Because the momentum transfer also scales with velocity, the duration of a complete collision process is independent of velocity!

There is only one potential that allows the duration of a period to be independent of velocity, which is the harmonic potential. Thus if one considers the motion of the centres of mass of the two spheres, they behave as if they exerted the force of a harmonic potential on each other. Confirmation of this conjecture is given in the next chapter by simulation results. This is of course quite different from what one would expect from Hertz' law of contact where the force is proportional to the separation to the power 3/2 (see App. C).

This result can have implications for fullerenes, e.g. $\mathrm{C}_{60}$ molecules: These molecules, consisting of 60 carbon atoms in a sphere-like structure, have a finite set of 174 vibrational modes $[\overline{\mathrm{ZZ}(0)}]$. If the interaction potential between two such molecules can be approximated by a hard-core potential, and if quantum effects can be neglected, it is predicted here that slow collisions of two fullerenes are harmonic.

\subsection{Summary}

In this chapter, the equations of motion for colliding, elastically vibrating bodies in one, two, and three dimensions were solved exactly under the assumption of an arbitrary but finite set of vibrational modes. The problem of collisions is thereby reduced from a set of continuum equations for each body plus hard-core interaction potentials between the bodies to the much simpler problem of finding the next points in space and time where the vibrating bodies will touch. Thus the time evolution is governed 
by simple rules on how to update linear and angular momenta and vibrations upon contacts, and by the equations for the free motion of the bodies inbetween contacts. The latter were derived for the case of arbitrary vibrating and rotating bodies. Such a scheme can easily be handled numerically, as will be demonstrated in the next chapter. 


\section{Three-dimensional particles: Simulations}

Using the results from the previous chapter, one can now perform simulations of the two particle collision process. With these simulations, it is finally possible to estimate the influence of the elastic vibrations on the collision properties of the particles and to test the influence of parameters like kinetic energy $E_{\text {kin }}$, angular momenta $\boldsymbol{L}_{i}$, impact parameter $b$, or the initial excitation of the modes $T_{i}$ (analogously defined as the bath temperatures of the rods in Ch. 2). Thus one can in principle calculate the analogue of $p_{\beta}(\epsilon)$ from Sec. 2.2 in three dimensions, if only numerically. This analogue is more complicated here since it is a function of (at least) two variables and with several parameters:

$$
p_{E_{\text {kin }}, \boldsymbol{L}_{1}, \boldsymbol{L}_{2}, T_{1}, T_{2}, b}(\epsilon, \beta)=\left\{\begin{array}{l}
\text { Probability density of finding a restitution coef- } \\
\text { ficient } \epsilon \text { and a roughness coefficient } \beta, \text { given the } \\
\text { initial conditions } E_{\text {kin }}, \boldsymbol{L}_{i}, T_{i}, \text { and } b
\end{array} .\right.
$$

The effective roughness parameter $\beta$ [ is the analogue of $\epsilon$ for the tangential velocity component: In many publications on these topics (see e.g. [JR8.56, Lun.91, GS.95, H7.97, LHM7.98, ML.98, HA799, AHZ00]), collisions are modelled as instantaneous using the collision laws

$$
\begin{aligned}
\boldsymbol{V}^{\prime} \boldsymbol{n} & =-\epsilon \boldsymbol{V} \boldsymbol{n} \quad \text { and } \\
\boldsymbol{V}^{\prime} \times \boldsymbol{n} & =-\beta \boldsymbol{V} \times \boldsymbol{n},
\end{aligned}
$$

where $\boldsymbol{V}$ is the relative velocity of the points of contact at the time of contact and $\boldsymbol{n}$ is the surface normal at the point of contact? The primed quantities on the lhs refer to the values after the collision, while the unprimed quantities on the rhs refer

${ }^{1}$ Unfortunately, the standard notation in the literature for this parameter is the same symbol that was used in Ch. 2 for the inverse bath temperature of the rods. In this chapter, I will use $\beta$ for the roughness parameter.

${ }^{2} \mathrm{Eq}$. (5.2) is enough to determine the collision completely if one also takes into account conservation of linear and angular momentum. The resulting equations and a discussion thereof with regards to statistical mechanics of granular particles can be found in [AHZ(0)]. 
to the values before collision. Thus in the same way that the coefficient of restitution became a stochastic variable in $\mathrm{Ch}$. 2, the roughness parameter $\beta$ becomes stochastic in this context. In principle one could even formulate Eqs. (5.1) and (5.2) in terms of two roughness parameters by introducing a basis in the plane defined by $\boldsymbol{n}$ and using two different parameters $\beta_{1}$ and $\beta_{2}$ along these basis vectors [Bro.96]. However, this will not be done here.

The computation of the complete probability distribution is beyond the scope of this work, but the purpose of this chapter is to introduce the algorithm and to show some first results of the simulations. The simulations are performed on a system of two homogeneous spheres since the normal modes of spheres are known and comparison with experiments is easiest.

\subsection{Vibrational modes of a sphere}

In this section, I will summarize the main results of [Lam82] for the vibrational modes of a homogeneous sphere.

Consider a homogeneous sphere of radius $R$ and mass $M$ (and resulting density $\rho=3 M / 4 \pi R^{3}$ ) which is made of a material with Poisson number $\sigma$ and transversal sound velocity $c_{\mathrm{t}}$ (see e.g. [L. [.91a] for a definition of $\sigma$ and $c_{\mathrm{t}}$ ). The second elastic modulus besides $\sigma$ that is needed to completely specify the elastic properties of a homogeneous, isotropic solid, e.g. Young's modulus $Y$, is contained in the definition of $c_{\mathrm{t}}=\sqrt{Y / 2 \rho(\sigma+1)}$. The actual shape of the modes of such a sphere depends exclusively on $\sigma$ while $R, M$, and $c_{\mathrm{t}}$ serve only as scaling factors. The modes fall into two distinct classes, the toroidal ones, $\boldsymbol{u}_{n m l}^{\text {tor }}(\boldsymbol{x})$, and the spheroidal ones, $\boldsymbol{u}_{n m l}(\boldsymbol{x})$ (without any superscript for notational ease). The former are purely transversal vibrations, the displacement $\boldsymbol{u}_{n m l}^{\text {tor }}(\boldsymbol{x})$ being everywhere orthogonal to the vector $\boldsymbol{x}$ that points from the center of the sphere to a point within the undeformed sphere. The toroidal modes appear to be less important than the spheroidal ones because Eq. (4.33) shows that the excitation of the modes by a collision is proportional to $\boldsymbol{n u}(\boldsymbol{x})$ which is usually small for the transversal vibrations since the surface normal $\boldsymbol{n}$ is almost parallel to $\boldsymbol{x}$ for small deformations. In the simulations, I will therefore concentrate on spheroidal modes only.

The explicit form of the modes is too long to be included here. A few remarks are in order for the indices $n, m$, and $l: n=0,1,2, \ldots$ is the order of the mode, analogous to the angular quantum number. It determines the degree of fineness of the structure on the surface, see Fig. 5.1. $m=-n,-n+1, \ldots, n-1, n$ is analogous to the magnetic quantum number. The numbers $n$ and $m$ appear as index of the well known spherical harmonics $Y_{n m}(\theta, \phi)$. The index $l=1,2,3, \ldots$ enumerates the infinitely many positive zeroes $k_{n l}$ of a certain function $P_{n}(k)^{3}$. The frequency of a mode is given by $\omega_{n m l}=k_{n l} c_{\mathrm{t}} / R$ and thus does not depend on $m$.

\footnotetext{
${ }^{3}$ For easier reference, this function is $P_{n}=B_{n} / A_{n}-D_{n} / C_{n}$ in Lamb's notation in [Lam82].
} 


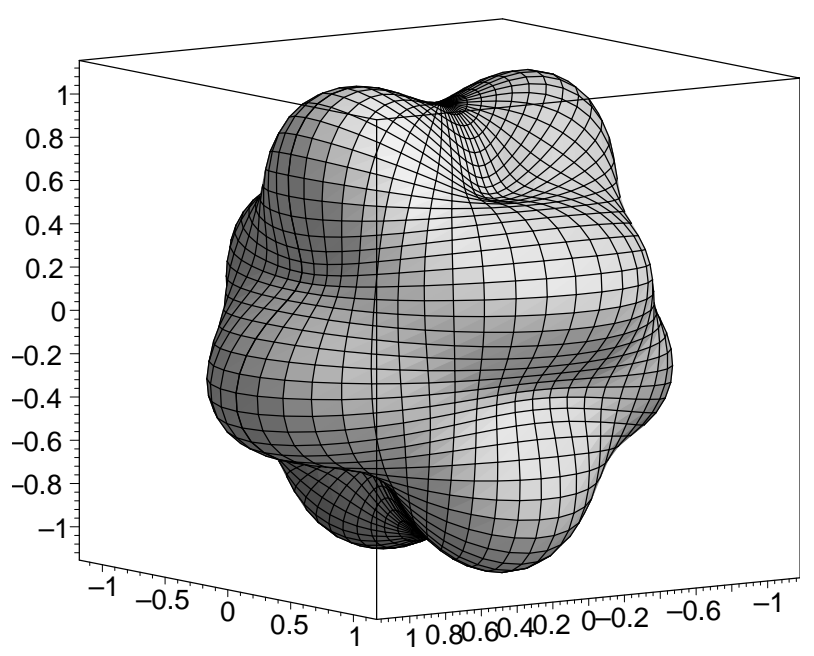

Figure 5.1.: The surface of a sphere with Poisson number $\sigma=1 / 3$ where the mode $\boldsymbol{u}_{521}$ is strongly excited. Note the five-fold symmetry which is due to $n=5$.

In the simulations, one must restrict oneself to a subset of the infinite set of modes. It proved useful to choose an upper limit $\omega_{\max }$ for the frequencies and to use all modes with $\omega_{n m l} \leq \omega_{\max }$. For ease of reference, such a set of modes will be denoted as $\Omega_{\omega_{\max }}$, i.e.

$$
\Omega_{\omega_{\max }}=\left\{(n, m, l) \mid \omega_{n m l} \leq \omega_{\max }\right\}
$$

Sometimes there will also be a limit on $n$, i.e. $n \leq n_{\max }$; such a set of modes will analogously be denoted by $\Omega_{\omega_{\max }, n_{\max }}$. I used a value of $\sigma=1 / 3$ (which is close to the value for steel) for the Poisson number and $\omega_{\max }$ up to $25 c_{\mathrm{t}} / R$ in the simulations. Fig. 5.1 shows for illustration how the surface of a sphere is deformed if one particular mode is excited.

\subsection{Computational methods}

As always, it is useful to use dimensionless units for the computations. Let the two spheres whose collision is going to be simulated have radii $R_{1}$ and $R_{2}$, masses $M_{1}$ and $M_{2}$ and transversal sound velocities $c_{\mathrm{t} 1}$ and $c_{\mathrm{t} 2}$, respectively. From these input parameters one can construct the following system of units: Lengths will be measured in units of

$$
L=\frac{2 R_{1} R_{2}}{R_{1}+R_{2}}
$$


the unit of velocity is

$$
c=\frac{2 c_{\mathrm{t} 1} c_{\mathrm{t} 2}}{c_{\mathrm{t} 1}+c_{\mathrm{t} 2}}
$$

hence the unit of time is $L / c$. Finally, the unit of mass $M$ is twice the effective mass $\mu$,

$$
M=2 \mu=\frac{2 M_{1} M_{2}}{M_{1}+M_{2}} .
$$

All simulation results presented below will be given in these units.

The algorithm to simulate a collision process is now straightforward:

1. Specify initial conditions: distance, relative velocitiy, angular momenta, excitation of the modes. It was already remarked earlier that under special symmetric conditions there may be more than one point of contact at one time. In order to avoid this (as the method can only handle single points of contact), the spheres must be initialised with tiny random excitations already before the collision.

2. Numerically solve the differential equations for the freely flying, vibrating spheres for a small time interval. These equations were derived in Sec. 4.5.

3. Find the minimum distance between the two deformed surfaces by picking two starting points, one on each surface, and moving them on their respective surfaces until their distance reaches a minimum. Repeat a few times for different starting points in order to increase the probability of finding the global minimum. Since there is no known algorithm that is guaranteed to find the global minimum of a complicated function, one has to resort to this rather unsatisfactory method. On the other hand, the space over which to find the minimum is only four-dimensional (two points on two-dimensional surfaces) and compact such that the structure of the minima is expected to be much less complicated than e.g. the energy landscape of structural glasses (see e.g. [Bha.9.]). Therefore one can hope that this simple method yields reasonable results. In fact, the simulations show that indeed it works very well if the starting points are chosen from a regular grid which is centered around the contact area.

4. If the minimum distance calculated in the previous step is smaller than a suitably chosen threshold $\left(\mathcal{O}\left(10^{-6}\right)\right.$ proved to be useful $)$, consider the particles as overlapping. The minimum distance is never negative, therefore it is no use trying to look for the point where it is exactly zero: due to numerical errors this will never be the case. Hence the threshold has to be introduced.

5. If the particles are overlapping, go backwards in time by solving the equations for the freely flying particles for a small negative time interval. Again, calculate 
the minimum distance at this time and check if it is above or below the threshold. Thus by going backward and forward in time by ever smaller amounts, one can "bracket" (see [PTVF92] for the method of bracketing e.g. the root of a function) the point in time where the particles first touch. This can be done up to a specified tolerance (e.g. $\left.\mathcal{O}\left(10^{-5}\right)\right)$.

6. When the point in time has been identified where the particles touch for the first time, Eqs. (4.20), (4.21) and (4.33) derived in the previous chapter can be applied to update the current values of linear and angular momenta and the excitations of the modes. The vector $\boldsymbol{n}$, the surface normal at the point of contact, is needed for this purpose. It has to be calculated by differentiating the surface (given by setting the radial coordinate $r=1$ ) with respect to the spherical coordinates $\theta$ and $\phi: \boldsymbol{n} \propto \partial\left(\boldsymbol{x}^{\prime}+\boldsymbol{u}^{\prime}\left(\boldsymbol{x}^{\prime}\right)\right) /\left.\partial \theta\right|_{r=1} \times \partial\left(\boldsymbol{x}^{\prime}+\boldsymbol{u}^{\prime}\left(\boldsymbol{x}^{\prime}\right)\right) /\left.\partial \phi\right|_{r=1}$ where $\boldsymbol{u}^{\prime}\left(\boldsymbol{x}^{\prime}\right)$ is the present displacement of the point $\boldsymbol{x}^{\prime}$.

7. Continue with step 2.

There exists the possibility to overlook a collision if the two spheres pass through each other during step 2 and have seperated again at the end of it. The only way to minimise this risk is to choose a small enough time interval. It has proved useful to choose about $1 / 10$ of the period of the fastest mode that is used.

The algorithm outlined above is the general procedure for simulations of collisions. Due to the fact that rotations are only extremely weakly excited, as will be demonstrated below, their mutual interaction with the vibrations has been neglected in the simulations that were actually performed. To be more precise, only the zeroth order approximation, Eq. (4.32), has been used. This results in a much simplified and accelerated code. In principle, however, one could follow Sec. 4.5 and couple rotations and vibrations.

\subsection{Simulation results}

\subsubsection{Preliminary observations}

The general geometry of a collision in a center of mass frame is shown in Fig. 5.2. The spheres collide with relative velocity $\boldsymbol{v}$. In a head-on collision, the impact parameter $b$ equals zero, otherwise $b$ differs from zero.

Fig. 5.3 shows a sample collision process of two identical, initially only weakly vibrating (in order to break the symmetry, as mentioned above) spheres in a head-on collision. The coefficient of restitution is very close to one in this run (the velocity curve comes up to almost its negative starting value). The number of modes used was rather small (see figure caption), therefore one might expect that the arguments given in Sec. 4.6 apply. There it was argued that for too small a number of modes no 


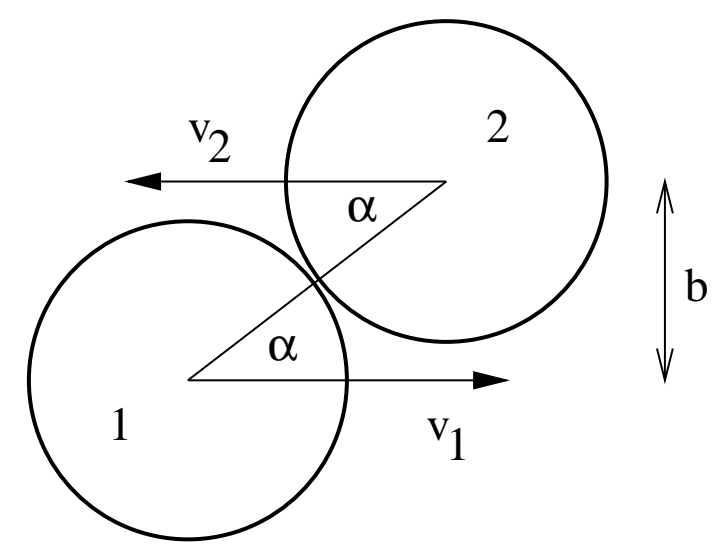

Figure 5.2.: Geometry of a collision and definition of the impact parameter $b$. The relative velocity is given by the difference of the individual velocities, $\boldsymbol{v}=\boldsymbol{v}_{2}-\boldsymbol{v}_{1}$.

contact face develops; instead the spheres always touch at the same point, resulting in not Hertzian but harmonic behaviour. This is indeed the case, as shown in Fig. 5.4. The figure shows the approximately linear relationship between the square of the operlap, $\delta^{2}:=(2-R)^{2}$ (only defined when the center of mass distance $R=|\boldsymbol{R}|$ is less than the sum $R_{1}+R_{2}=2$ of the radii of the two spheres), and the "potential" energy. Potential energy here means $E_{\text {tot }}-E_{\text {kin }}$, i.e. the energy that is missing from the centre of mass kinetic energy. It is written in quotes to indicate that it is a truly potential energy only in the quasistatic case while in general it also contains the kinetic energy of the vibrations. A linear relationship as shown in this figure is what one expects for a harmonic potential while for instance Hertz' law of contact would result in the elastic energy being proportional to $\delta^{5 / 2}$ (refer to App. C for a short overview over the results of Hertz' contact theory). Thus the conjecture made in Sec. 4.6 that for low velocities the spheres behave harmonically is verified by this simulation.

It was stated in Sec. 4.6 that the duration of a collision should be independent of velocity for small velocities. Thus an additional test of the arguments brought forward in Sec. 4.6 is the calculation of the duration of collisions at different initial velocities. This is exemplified in Fig. 5.5 for two initial velocities which differ by a factor of 10. Note that there is no fitting involved; the only scaling that was required to obtain matching curves was the scaling of velocities by their initial value. If Hertz' law were valid here, the durations would differ by a factor of $10^{1 / 5} \approx 1.58$.

Fig. 5.6 shows how one of the two spheres is deformed at the time of closest approach. It is only very slightly indented on the right and retains an almost spherical shape which again confirms that contact of the two spheres only occurs at one point.

The observations made so far indicate that a) the arguments in Sec. 4.6 were correct and b) that either the number of modes or the initial velocity has so far 


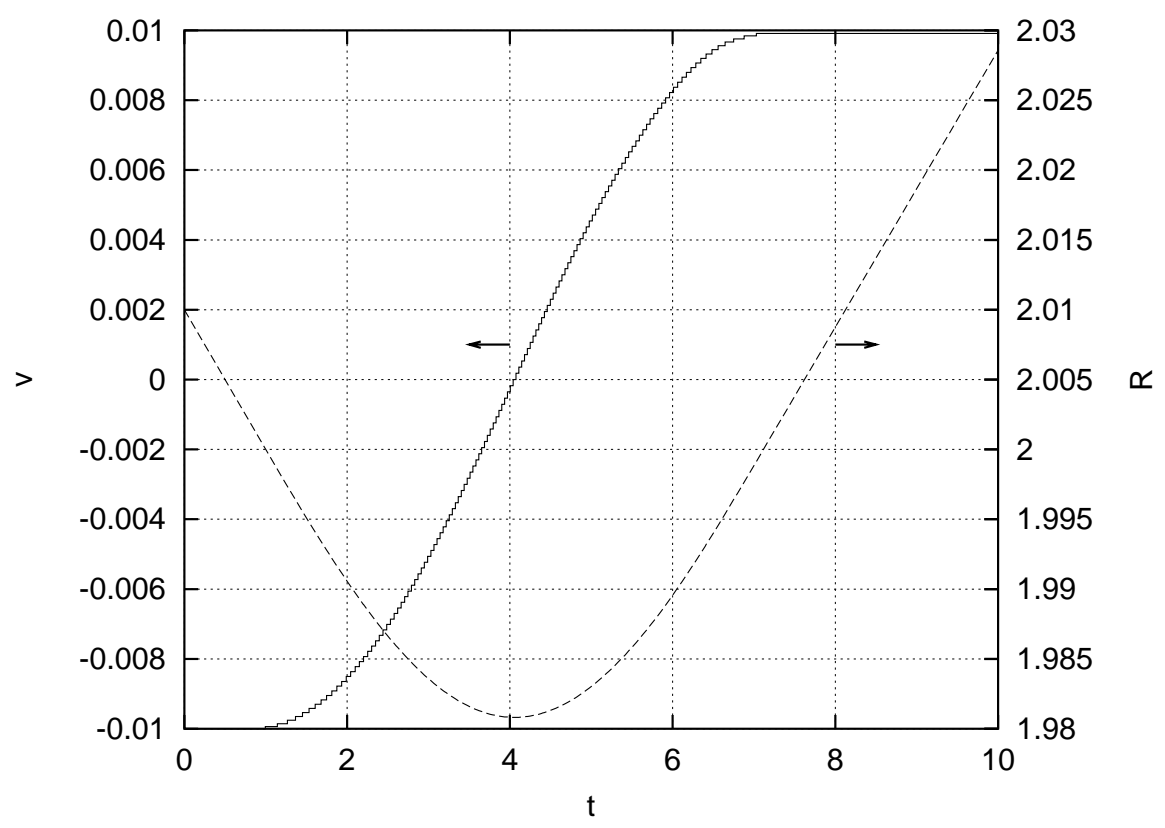

Figure 5.3.: Head-on collision process of two identical, initially barely vibrating spheres. Modes with $n \leq 10$ and $\omega \leq 25$ (dimensionless units), i.e. the set $\Omega_{25,10}$ (cf. Eq. (5.3) and following text), were used for this run. The solid curve shows velocity vs. time (left axis). The small steps that can be identified in the velocity curve are due to the $\delta$-collisions. The distance of the centers of mass is shown by the dashed curve (right axis).

been too small to yield results comparable to a continuum theory such as Hertz'. Therefore I will now turn to higher velocities and/or larger numbers of modes.

\subsubsection{Simulations with more modes and higher velocities}

\section{Comparison with Hertz' contact law}

In order to test whether the simulations show better agreement with continuum theory when the number of modes is increased, head-on collisions with $v(0)=-0.02$ using three different sets of modes were carried out. Fig. 5.7 shows the potential energy as a function of the overlap for these three sets of modes $\Omega_{15}, \Omega_{20}$, and $\Omega_{25}$, along with the theoretical curve from Hertz' calculation (see App. C, Eq. (C.4)). Evidently, the spheres become softer when the number of modes is increased and the curves seem to approach the Hertzian result, even though the agreement is not yet very good.

However, there is a qualitative difference between the $\Omega_{15}$-curve in Fig. 5.7 and the other two: While the upper curve is approximately quadratic in $\delta$ (just as the 


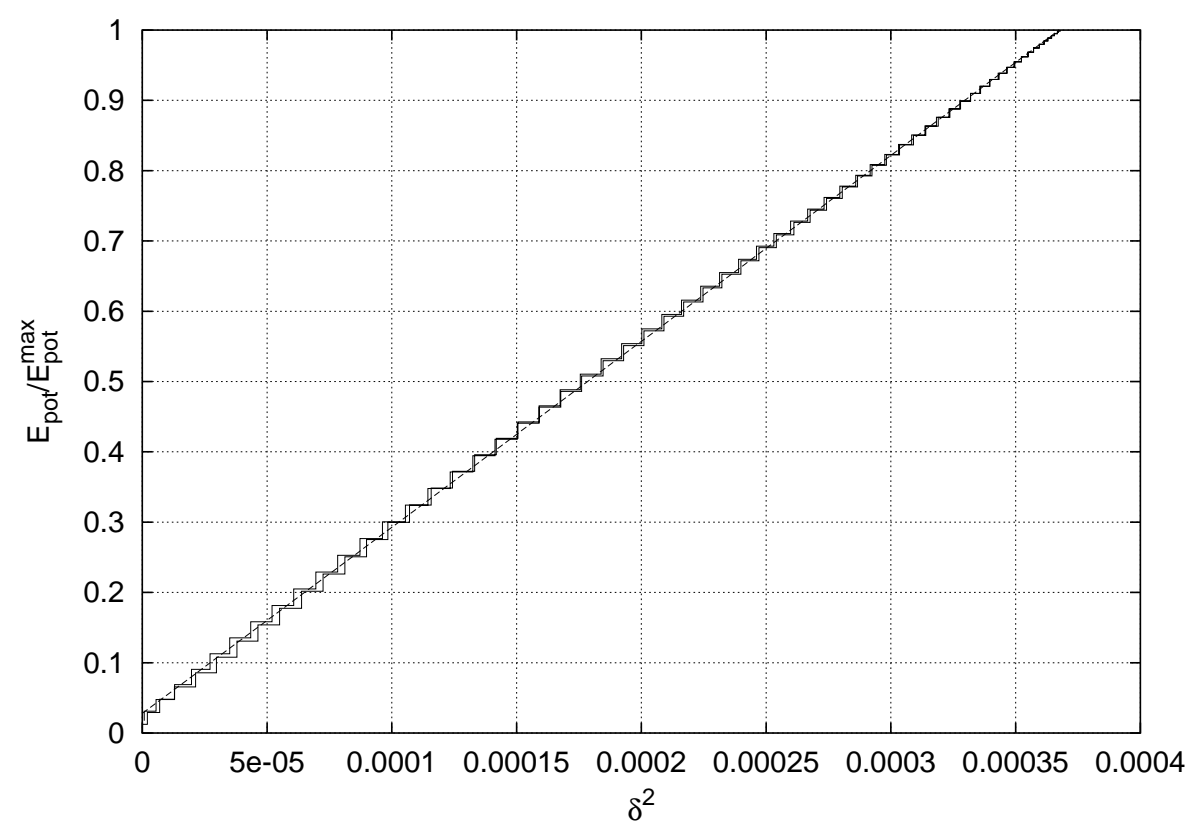

Figure 5.4.: The linear relationship between the sqare of the overlap, $\delta^{2}=(2-R)^{2}$, and the normalised potential energy for the same run as in Fig. 5.3. The straight line is a best fit for illustration. Evidently the spheres behave as if in a harmonic potential. See text for details.

one in Fig. 5.4), the other curves start to cross over to a higher power. This can best be seen on a logarithmic plot, Fig. 5.8. While the upper curve is well parallel to the $\delta^{2}$ line, clearly the curve for $\Omega_{25}$ deviates from it and develops a slope which is more like $5 / 2$ as in Hertz' law. The $\Omega_{20}$-curve moves between these two, starting off like the upper one but then deviating from it and coming closer to the lower one. At the end, its slope seems to be slightly higher than 2. Of course such a logarithmic plot can only give a hint of the true exponents since there are far too few decades available in order to draw a decisive conclusion. It shows, however, that the results are consistent with Hertz' theory.

The fact that there is a qualitative difference between the three curves is also indicated by the "fine structure" of the curves: While the upper curve has very regularly spaced steps, the lower two are much more irregular. This is due to the development of the contact face since collisions on different parts of the contact face can occur in very rapid and irregular succession. In contrast, the collisions are very regularly spaced if no contact face develops (due to a small number of modes) and the spheres always touch at the same point.

For further comparison with Hertz' theory, the contact durations for twot of

\footnotetext{
${ }^{4}$ The third was not yet available at the time of this writing.
} 


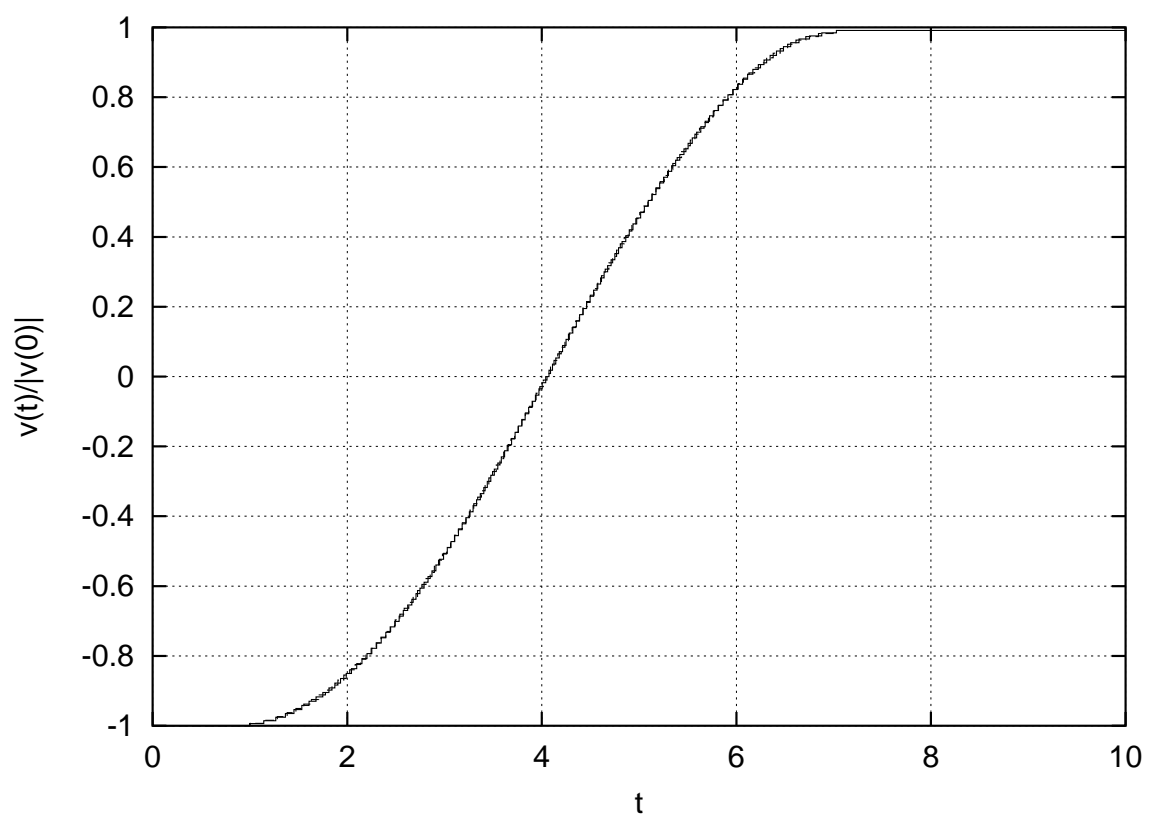

Figure 5.5.: Head-on collisions of identical spheres with different initial velocities. The solid line is for an initial velocity of $v(0)=-0.01$, the dashed line for $v(0)=-0.001$. The two curves are basically indistinguishable, confirming the indepence of the duration of the collision of initial velocity. The same set of modes as in Fig. 5.3 was used.

the three sets of modes and the quasistatic case according to Eq. (C.5) have been computed and collected in Table 5.1. The durations were calculated by taking the difference of the two times at which the centre of mass distance is equal to the sum of the radii. Again, increasing the number of modes shifts the contact durations towards the expected Hertzian result but agreement is still not very good.

It remains to be tested if Hertzian behviour should have been expected at the chosen impact velocity of 0.02 or if the kinetic energy of the vibrations is so high during the collision that it invalidates the assumptions of the quasistatic calculation. Fig. 5.9 shows, however, that the collision is basically quasistatic since the kinetic energy of the vibrations fluctuates around zero.

\section{Effects of increasing impact velocity}

As was shown in the previous section, a velocity of $v=0.02$ is small enough to lead to a quasistatic collision behaviour. The question arises, what happens if the impact velocity is increased such that non-negligible vibrational kinetic energies occur. In order to answer this question, simulations of head-on collisions with impact velocities 0.04, 0.08, 0.1, and 0.16 were carried out. To keep the computational burden low, 


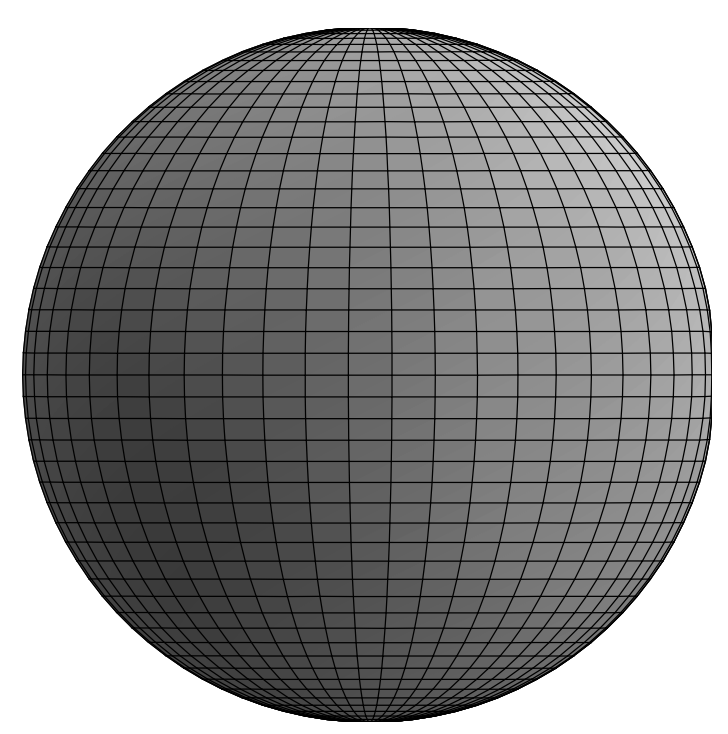

Figure 5.6.: Deformation of one sphere at the time of closest approach (the initial velocity was $v=-0.01$ ). The other sphere would be to the right of this one. On that side, a very slight deformation is visible. For this run, the set of modes $\Omega_{20}$ was used.

Table 5.1.: Contact times at impact velocity $v=0.02$ for two different sets of modes and according to Hertz' contact theory, Eq. (C.5).

\begin{tabular}{|l|l|}
\hline Set of modes & Contact duration (approx.) \\
\hline$\Omega_{15}$ & 6.77 \\
$\Omega_{20}$ & 7.08 \\
\hline Hertz & 8.23 \\
\hline
\end{tabular}

the set $\Omega_{15}$ was used for all collisions. It will be seen below that this set is large enough at these velocities to escape the harmonic behaviour. It is probably not large enough to produce quantitatively reliable results since for $v=0.02$, even $\Omega_{25}$ was not enough, but it allows for comparisons between the different runs. For illustration, Fig. 5.10 shows how a sphere is deformed at the point of closest approach for $v=0.1$. The contact face can be clearly distinguished.

First, let's look at the vibrational kinetic and potential energy during the collisions. The vibrational kinetic energy is an indicator for whether the collision is quasistatic or not. Fig. 5.11 shows that for all of the chosen impact velocities, the collision is not quasistatic since all the kinetic energy curves lie clearly above zero. Note the evident difference to the case where the impact velocity is 0.02, Fig. 5.9: there, the potential energy is indeed zero on average. However, even though there is a non-zero kinetic energy here, it is still small compared to the potential energy. 


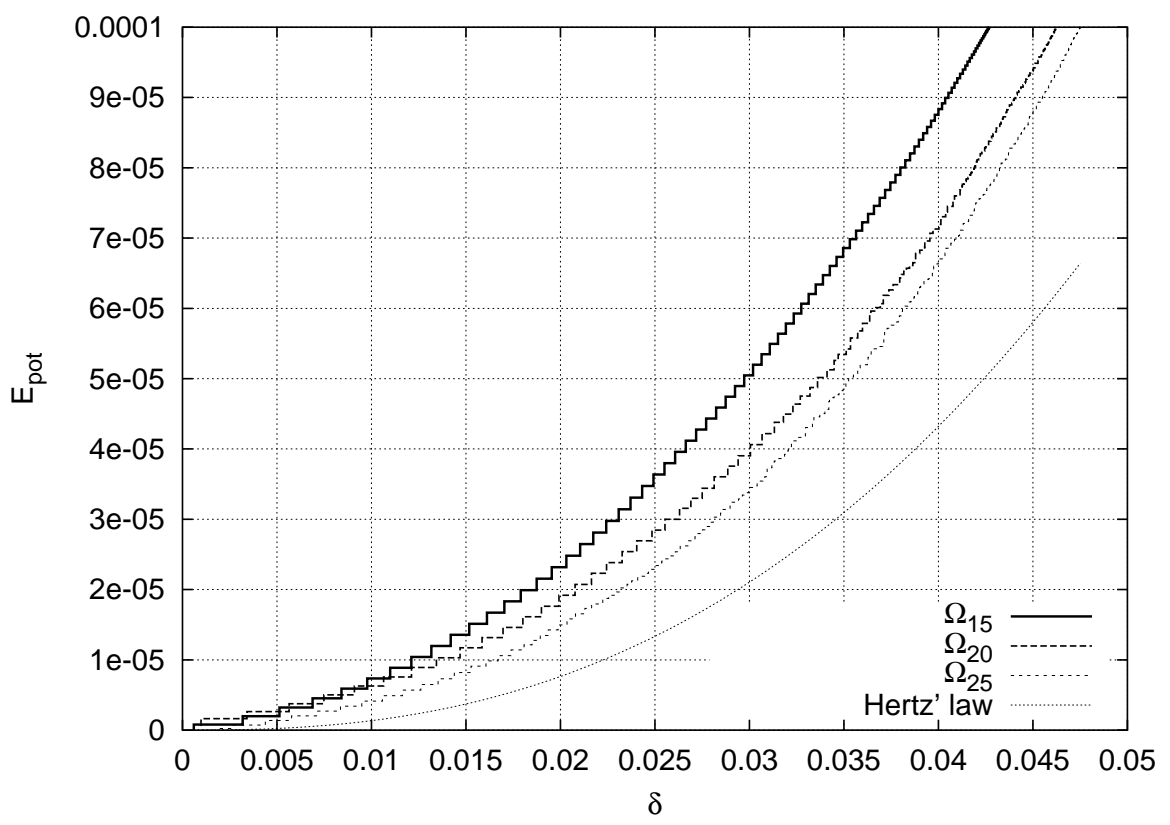

Figure 5.7.: Head-on collision of two spheres with initial velocity $v(0)=-0.02$. The "potential" energy is shown vs. the overlap $\delta$. The upper three curves correspond to three different sets of modes, $\Omega_{15}$ (containing a total of 393 modes), $\Omega_{20}$ (860 modes), and $\Omega_{25}$ (1555 modes). Only the approach of the spheres up the point of smallest distance is shown since the way back looks essentially identical. For comparison, Hertz' law is also shown.
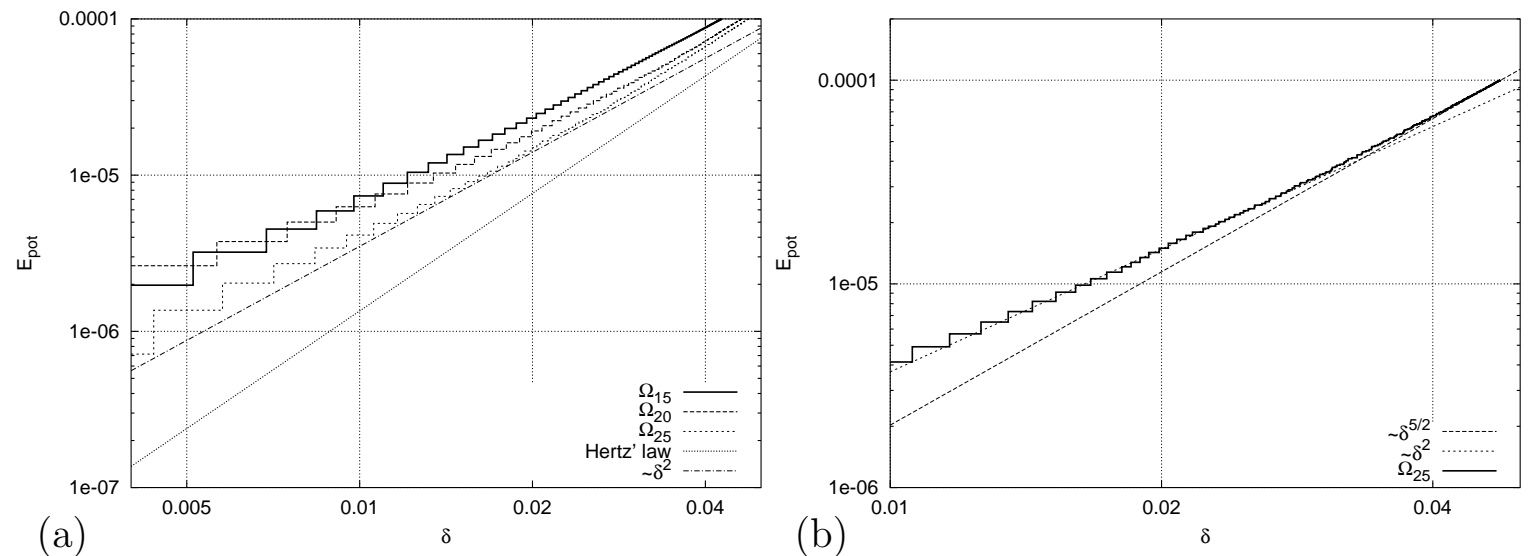

Figure 5.8.: (a) The same data as in Fig. 5.7 on a double logarithmic scale. Note the change in the slope as the number of modes increases. In the enlargement (b), the $\Omega_{25}$-curve is compared with two straight lines with slopes 2 and $5 / 2$, showing that it starts off as the former and then crosses over to the latter. 


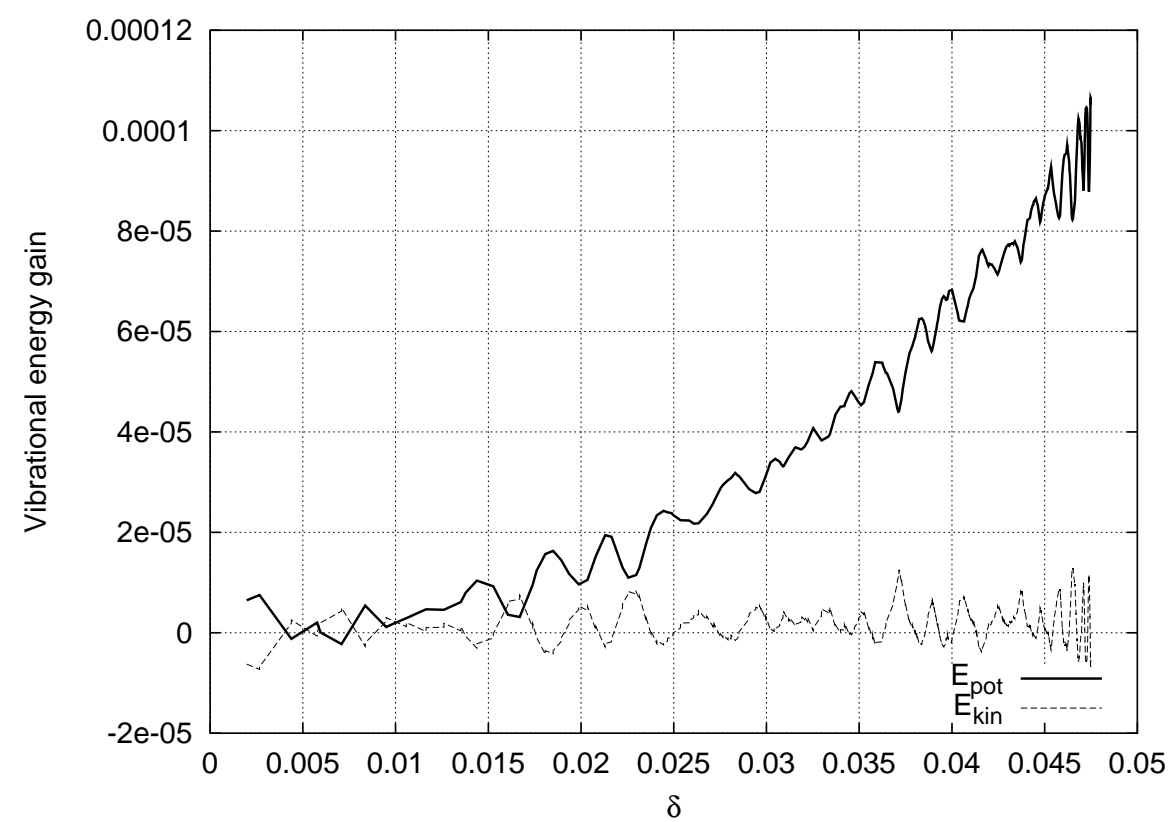

Figure 5.9.: The same collision as the one using $\Omega_{25}$ in Fig. 5.7. The seemingly negative energies come from the fact that the spheres are slightly vibrating even before the collision starts, while this plot only shows the energy gain with respect to the initial vibrational energy. The kinetic energy gain fluctuates around zero, indicating quasistatic behaviour.

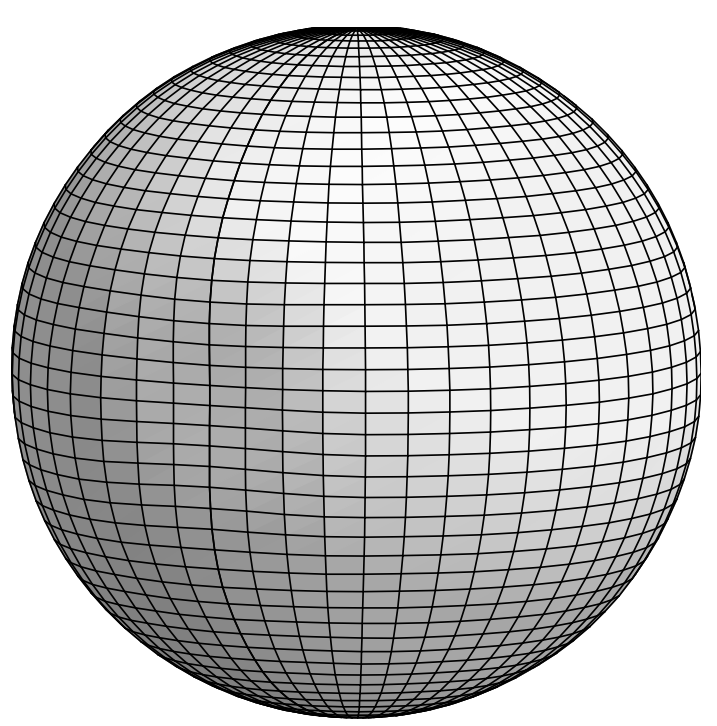

Figure 5.10.: Deformation of a sphere at the point of closest approach with impact velocity $v=0.1$. Note the clearly developed contact face. 


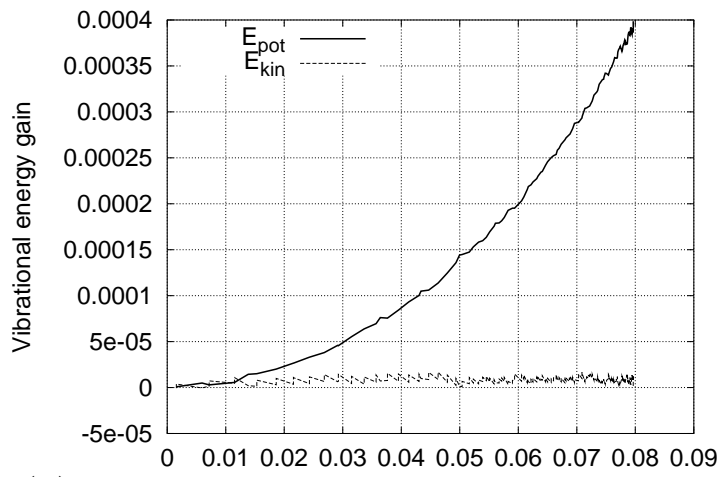

(a)

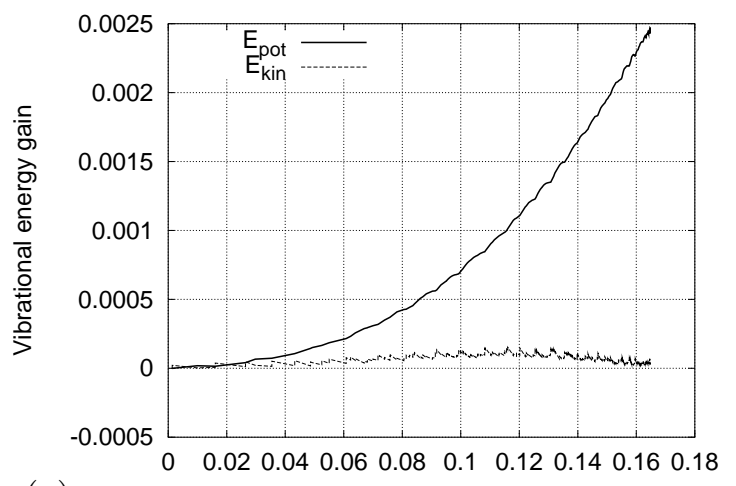

(c)

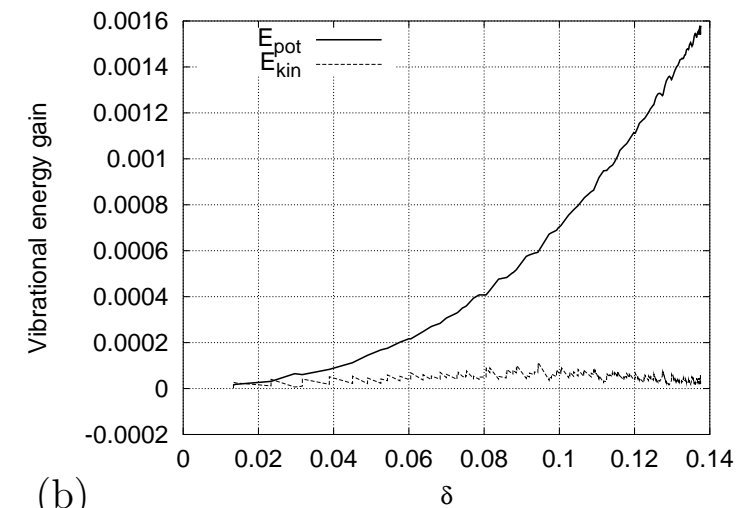

(b)

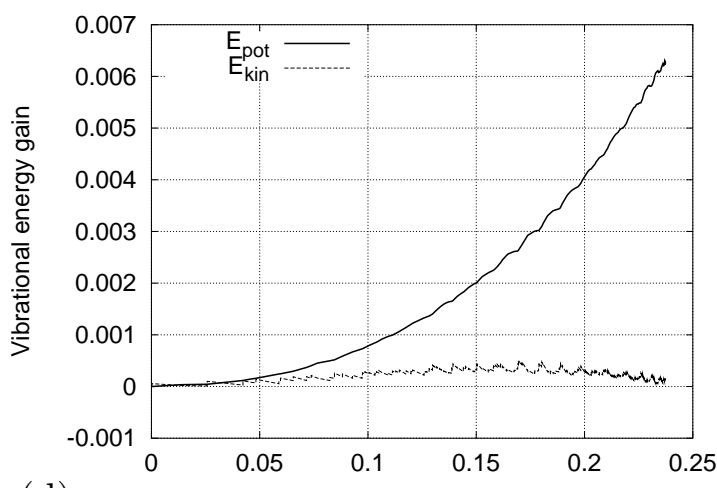

(d)

$\delta$

Figure 5.11.: Vibrational kinetic and potential energy as a function of the overlap $\delta$ for four different impact velocities: 0.04 (a), 0.08 (b), 0.1 (c), and 0.16 (d). In all runs, $\Omega_{15}$ was used. Note the difference in scales on both axes in all four plots. Again, only the approach of the spheres is shown. 


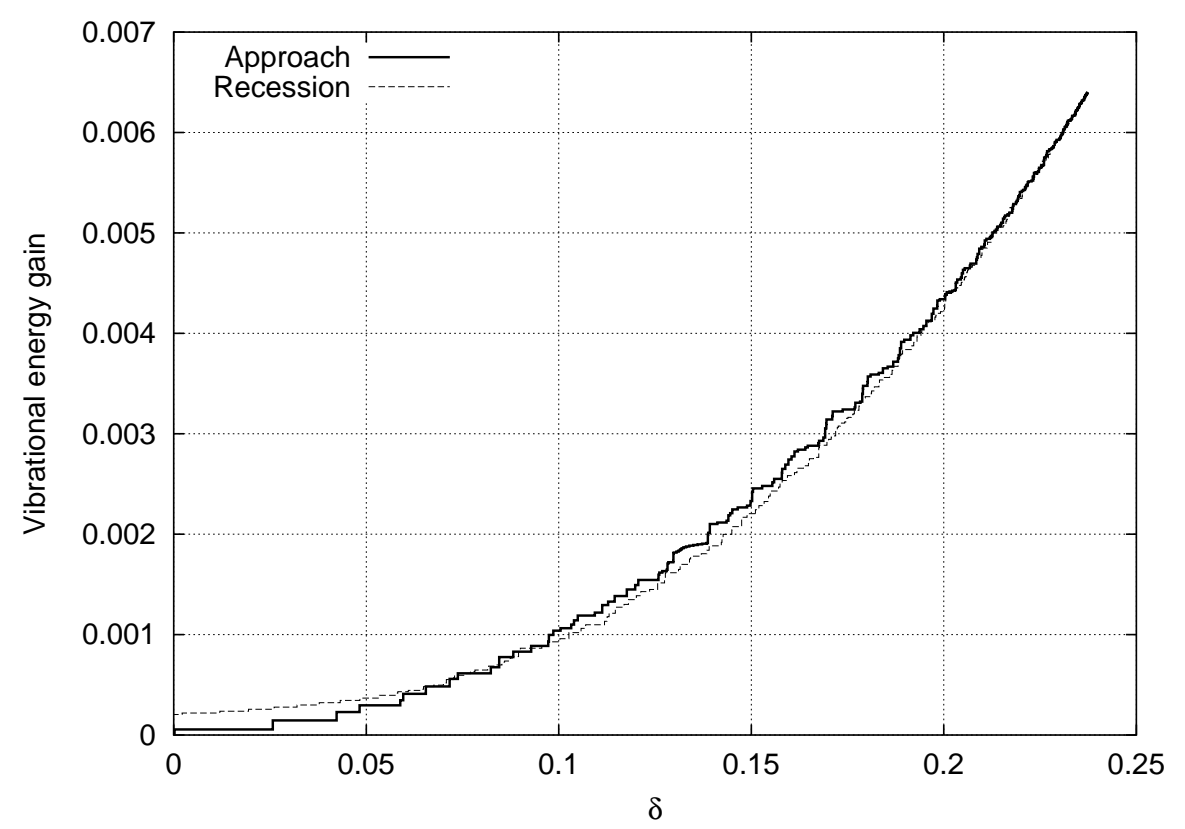

Figure 5.12.: The vibrational energy gain as a function of the overlap for impact velocity 0.16 . The curves for approach and recession differ.

While the potential energy looks fairly similar in all four plots, the kinetic energy develops a more and more pronounced bump as the impact velocity increases. Surprisingly, and unlike the potential energy, the maximum of the kinetic energy (at least for Figs. 5.11 (b)-(d)) does not lie at the point of closest approach but at about three quarters along the way. The reason for this is not known.

Another clear indicator that the collisions analysed in this section have left the quasistatic regime is the fact that the collisions are beginning to become unsymmetric: the approach of the spheres is different from their recession. This is exemplified by Fig. 5.12 which shows the vibrational energy as a function of the overlap $\delta$. The curves for approach and recession differ slightly, not only at around $\delta=0$, the beginning and end of the collision, but also during almost the entire process. Additionally, this figure shows the first hint of a collision where vibrations persist after the collision is over: The vibrational energy at the end of the recession curve is substantially higher than zero, meaning that the spheres are still vibrating. More of this is to follow below.

It was mentioned before that the kinetic energy of the vibrations is still small compared to the potential energy. This suggests that the quasistatic theory should still give reasonable results. In order to test this, the contact durations of the four runs analysed above were computed and compared with the theoretical ones. It can not be expected that they agree quantitatively since even at low impact velocities and using a large set of modes there is still a discrepancy (see Table 5.1). However, 


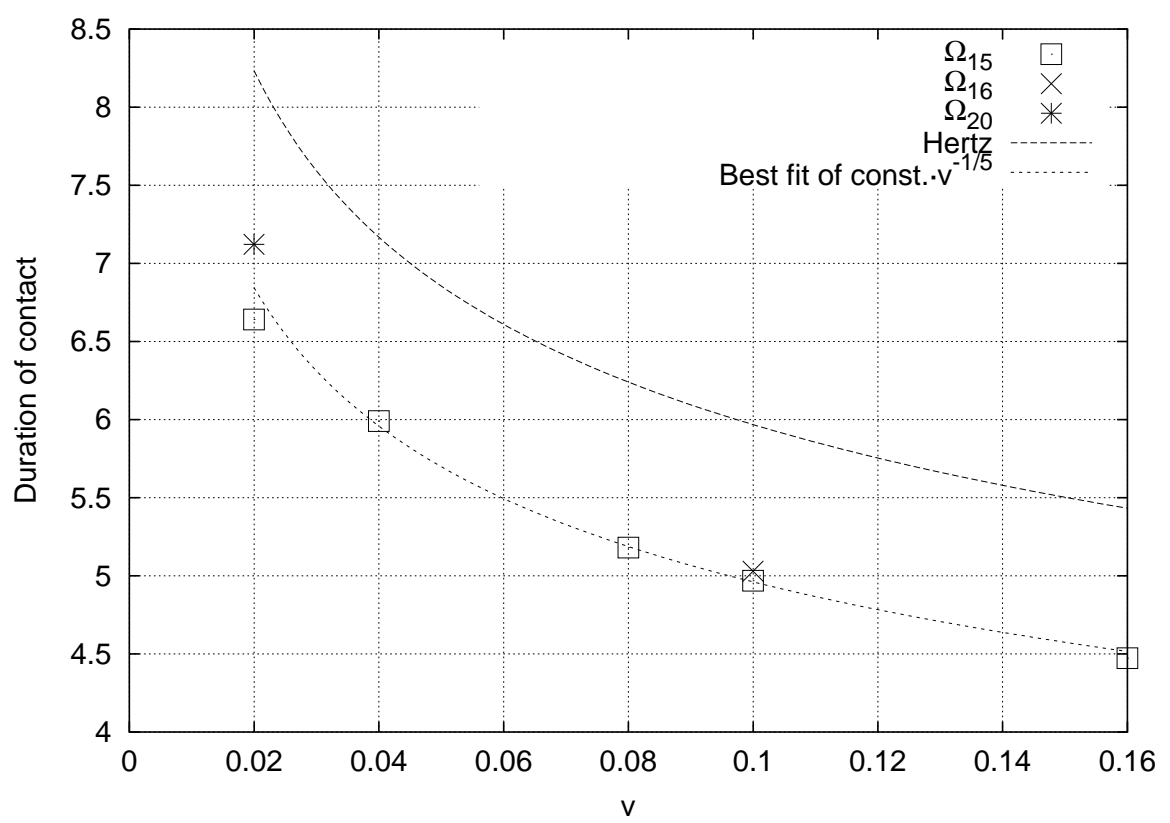

Figure 5.13.: The duration of a collision as a function of impact velocity. While all contact durations are too small compared to the theoretical result, they still nicely obey a power law $v^{-1 / 5}$, as the fitted line shows. The data points for $v=0.02$ lie off the curve since at that velocity, the number of modes is either too small $\left(\Omega_{15}\right)$ and the spheres behave harmonically or too large $\left(\Omega_{25}\right)$ such that the data is not comparable to the other points.

it can be tested if the contact duration is proportional to the impact velocity to the power $-1 / 5$ as predicted by Hertz' theory, Eq. (C.5). This is shown in Fig. 5.13. Of course there are not enough decades available for a thorough testing of the exponent $-1 / 5$, but the data is very well compatible with it.

Even though the vibrational kinetic energy is still small, it is surprising that Hertz' law (at least the exponent, if not the prefactor) describes the data so well. However, a similar phenomenon has been found experimentally [BL.71] where the authors report good agreement of their measurements of contact durations of colliding spheres with Hertz' theory even well outside its expected range of validity. They find exponents of -0.232 and -0.221 , depending on the material of the spheres.

It was seen in Fig. 5.12 that the vibrational energy gain is not zero at the end of a collision. This shows that collisions outside the quasistatic regime can indeed be inelastic in the sense that vibrations are excited by the collision. Fig. 5.14 shows the resulting coefficients of restitution $\epsilon$ as a function of impact velocity. While the $\epsilon$-values are still fairly close to 1 , they are nevertheless noticable. As expected, they decrease with impact velocity. Furthermore, it can be inferred from the two 


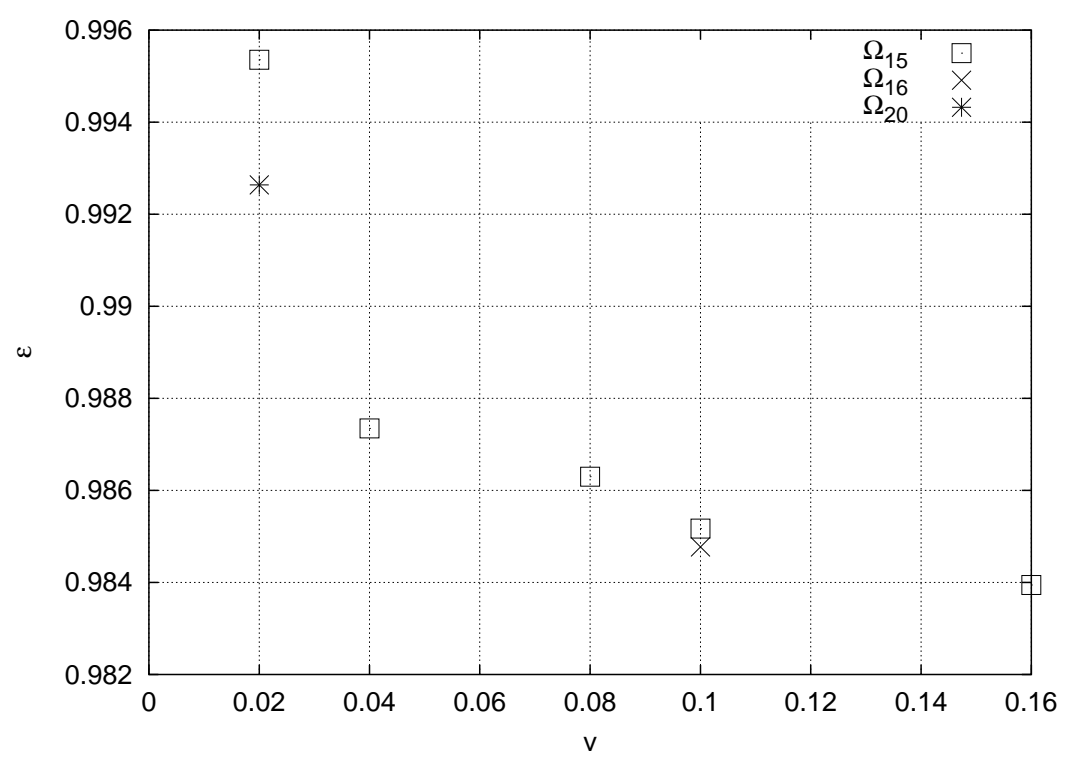

Figure 5.14.: The coefficient of restitution $\epsilon$ as a function of impact velocity $v$.

data points obtained with larger sets of modes that they seem to decrease with the number of modes used. This is particularly evident at $v=0.02$ : The run with $\Omega_{15}$ was shown above to behave harmonically, thus it has a coefficient of restitution very close to 1 , but the run using $\Omega_{20}$, which is more realistic, shows a significantly smaller $\epsilon \approx 0.9925$. This is in contrast to [G/9.9] where it is found that the coefficient of restitution increases with the number of modes for elastic two-dimensional disks.

\section{Non-head-on collisions}

Apart from the head-on collisions described in the last section, it is also possible to look at impact parameters $b \neq 0$.

First, it has to be checked if non-head-on collisions produce rotations of the spheres. If so, this would amount to an effective mechanism of friction. An example is shown if Fig. 5.15 for $b=1.5$ and impact velocity $v=0.1$. During the collision, rotations are produced since the spheres touch at various points on the contact face, each time generating a non-zero angular momentum. However, towards the end of the collision, the rotations cancel almost completely. Thus there is no effective friction. This behaviour is found for all $b$, and Fig. 5.15 is not special for grazing collisions: the respective curves look very similar for any $b$, even $b=0$.

Since there is no effective friction, it is enough to consider only the coefficient of normal restitution $\epsilon$, while the coefficient of tangential restitution, $\beta$, equals -1 . The coefficient of normal restitution as a function of the impact parameter is shown in Fig. 5.16. The variation in $\epsilon$ shows a peak at intermediate $b$. This is not very intuitive but is explained by looking at the ratio of translational kinetic energies before and 


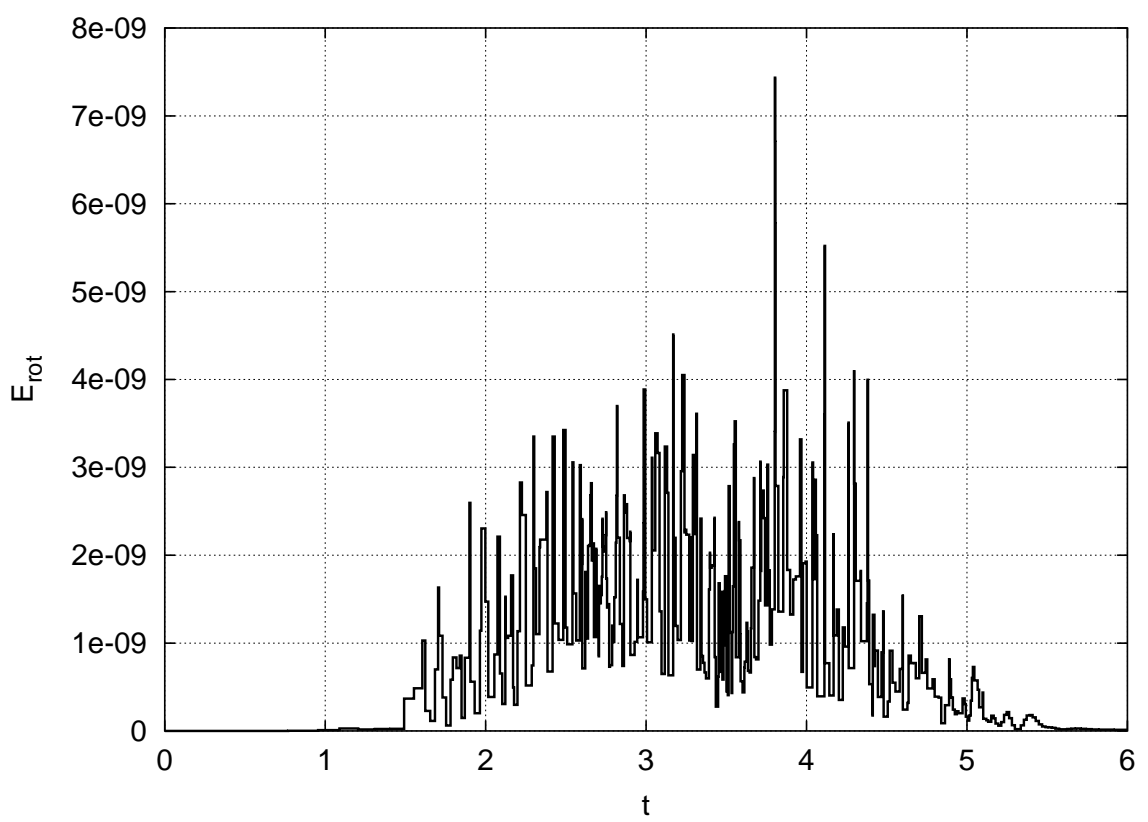

Figure 5.15.: Rotational energy of two colliding spheres (impact velocity $v=0.1$, impact parameter $b=1.5$ ) as a function of time. Rotations start to build up shortly after the collision has begun but cancel almost completely towards the end of the collision.

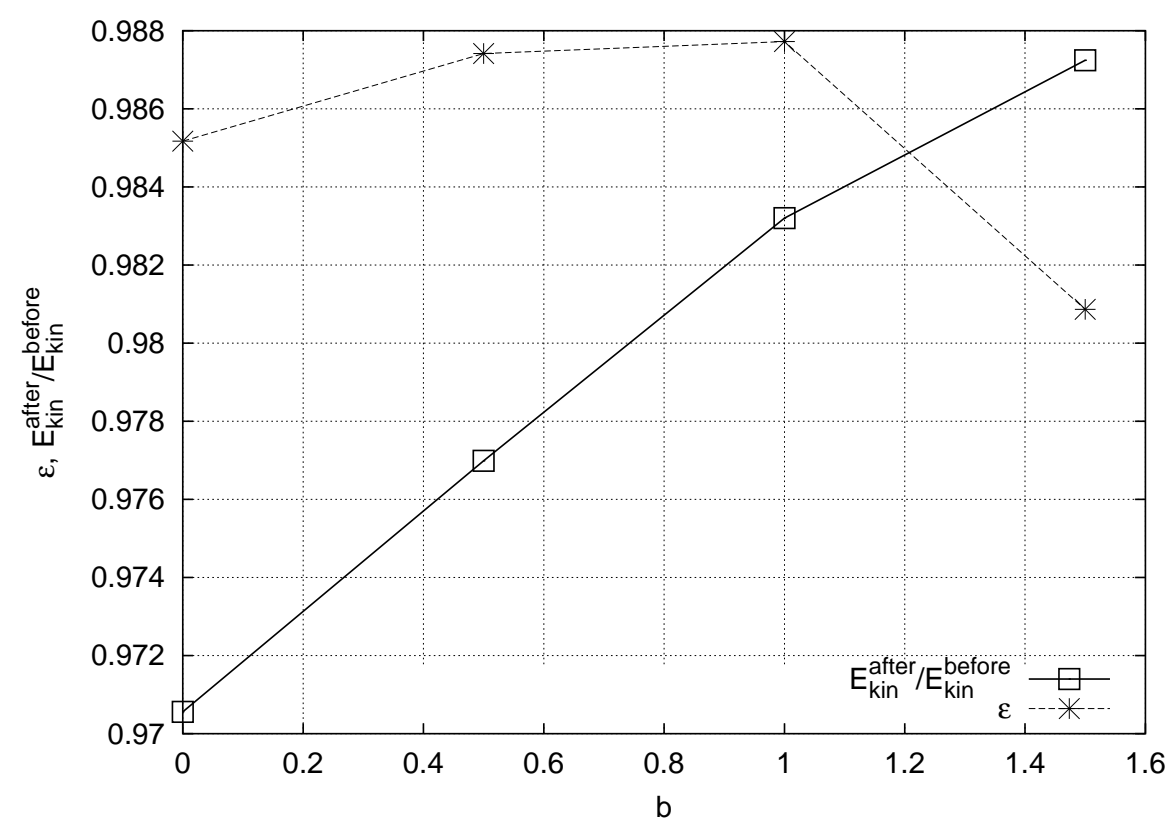

Figure 5.16.: The coefficient of normal restitution $\epsilon$ and the ratio of initial and final translational kinetic energy as a function of impact parameter $b$. 
after the collision. This ratio monotonically increases with $b$, which is understandable since grazing collisions should have a less severe impact on the spheres than headon collisions. The coefficient of restitution $\epsilon$, being the ratio of the initial and final normal velocity components, is first influenced by the decreasing energy loss, resulting in increasing $\epsilon$. For larger $b$, the initial normal velocity component becomes so small that even a small energy loss causes it to be greatly reduced.

\section{Spheres of unequal size}

The case of two identical spheres provides a good testing ground for the simulations but physically more interesting situations arise when the spheres have different sizes. It is known from the one-dimensional particles that a length ratio $\gamma$ smaller than one results in a coefficient of restitution $\epsilon=\gamma$ (see Sec. 2.2.2). Thus it might be conjectured that the coefficient of restitution will decrease for spheres of unequal size. The effect will be less pronounced in three dimensions than it is in one since in the low velocity limit it is known from Hertz' law that there is no energy loss in the quasistatic regime. A simulation of a head-on collision of two spheres using $\Omega_{20}$ with radii which differ by a factor of two is shown in Fig. 5.17. The impact velocity is $v=0.1$ (note that according to Sec. 5.2, changing the ratio of radii from 1 to $1 / 2$ changes the units of length $L$ and time $L / c$ but not the unit of velocity $c$ ). The larger of the two spheres gains more kinetic and potential energy during the collision than the smaller one, and it continues to vibrate after the collision is over. There seems to be essentially only one excited mode since the curve appears to be very much like a single sin-function. The period of vibration can be extracted from the figure and yields $T \approx 3.54$ which agrees very well with the period of the mode having the lowest frequency: The period of this mode turns out to be $T_{\text {slow }}=3.556 \ldots$. Thus it is primarily this mode which is excited in the simulation, a result which might have been expected since this lowest mode is the one which contracts (resp. expands) the sphere in the direction of approach and dilates (resp. contracts) it in the directions perpendicular to it, giving it a pancake-like (resp. cigar-like) appearance, depending of the phase of the oscillation. Fig. 5.18 shows the same data as Fig. 5.17 as a function of the overlap. This plot emphasizes the unsymmetric and non-quasistatic nature of the collision. Note that during a certain phase of the collision the vibrational kinetic energy of the larger sphere becomes actually larger by a factor of about two than the potential energy, indicating that the quasistatic approximation is definitely invalid for this situation.

The coefficient of restitution and the fractional translational energy loss which result from this simulation run are

$$
\epsilon=0.9645 \quad \text { and } \quad 1-\epsilon^{2}=0.06974
$$

These values are decidedly more inelastic than for identical spheres with the same 


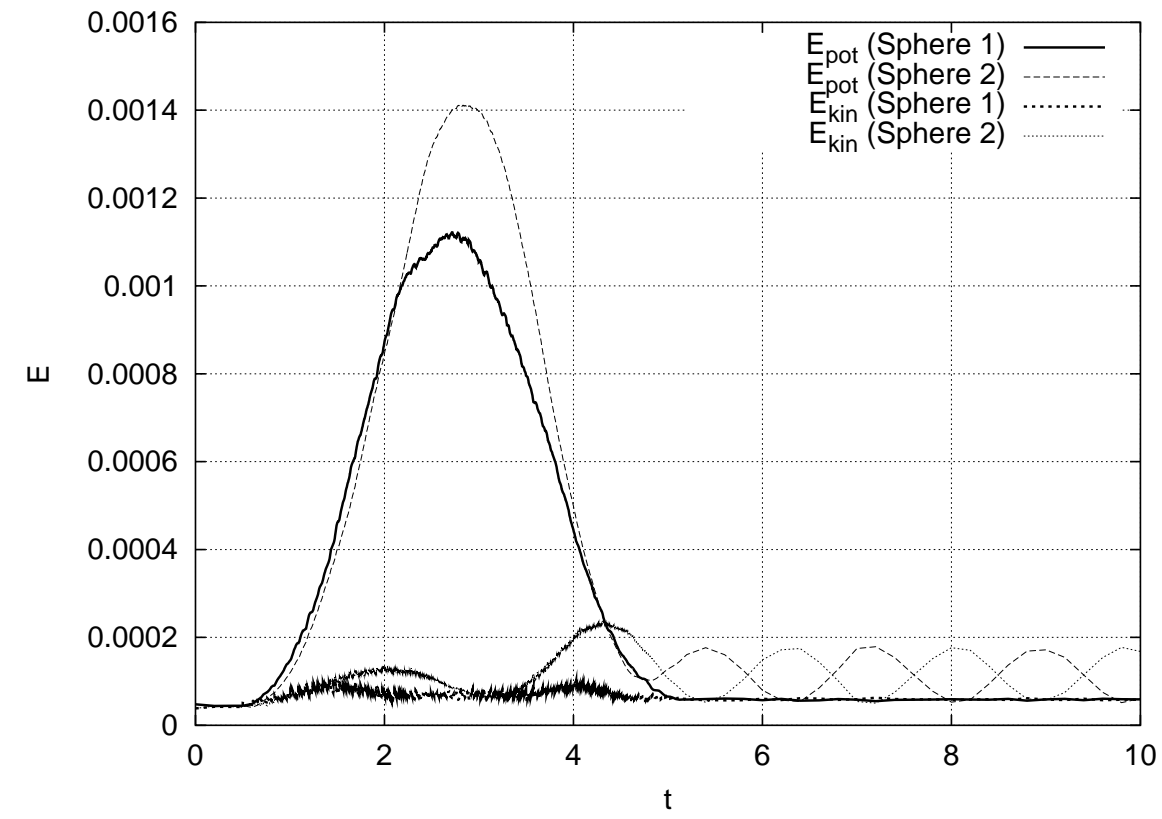

Figure 5.17.: Time evolution of the vibrational energy gains of two spheres of different radii $\left(R_{2}=2 R_{1}\right)$ using $\Omega_{20}$ in a head-on collision. The larger sphere gains more potential and kinetic energy than the smaller one, and there is a considerable fraction of energy that it retains after the collision is over. The smaller one shows no visible vibration after the collision.
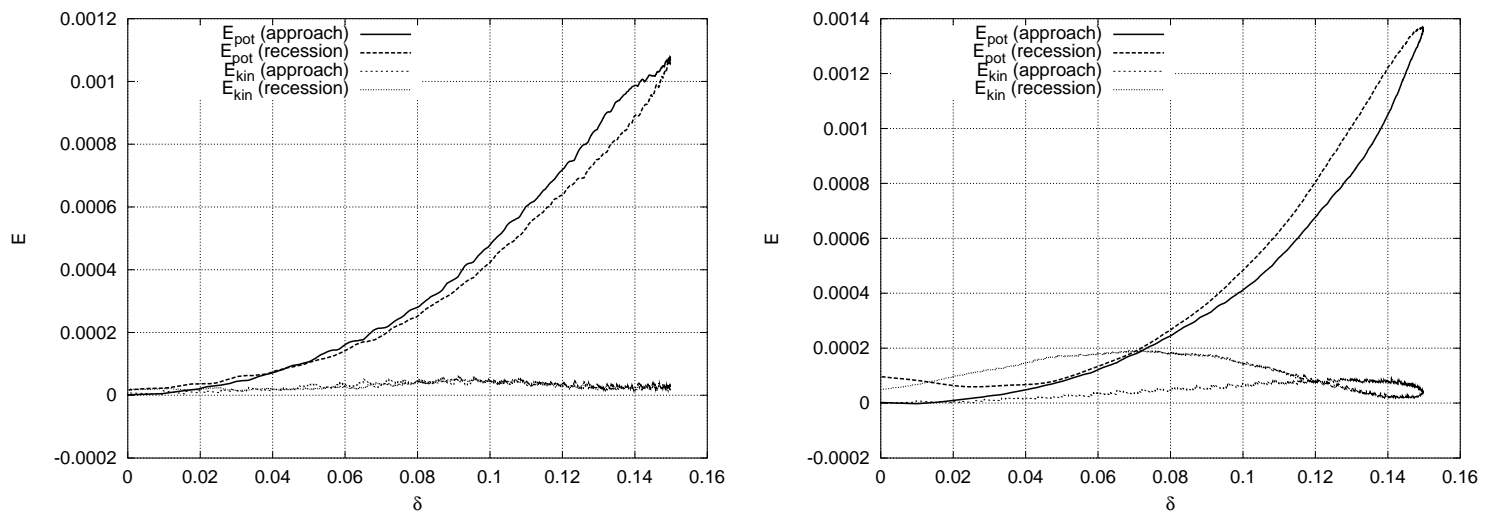

Figure 5.18.: The vibrational kinetic and potential energy gain as a function of the overlap for a ratio of radii of $1 / 2$. The collision is extremely unsymmetric with respect to time (or overlap), contrary to a quasistatic calculation. Although the impact velocity is even less than in Fig. 5.12, the unequal sizes change the behaviour drastically. 
impact velocity, where

$$
\epsilon=0.9848, \quad 1-\epsilon^{2}=0.03017
$$

was found (see Fig. 5.14]). Rayleigh calculated [Ray06] that the excitation of vibrations by collisions should be small for equal spheres. The simulations show, however, that his arguments can not simply be extended to the case of unequal spheres since the energy loss shown here for spheres of different radii is significantly higher than for equal spheres.

\subsection{Summary}

In this chapter, the simulation method that was made possible by the calculation in Ch. 4 has been described in detail. It provides a tool to compute the macroscopic collision behaviour (e.g. coefficients of restitution) of two bodies, based on a microscopic model. Some first results of applying the method to collisions of spheres have been presented.

The main findings are that the method works, that it produces results which can be compared to Hertz' theory of contact (although the quantitative agreement is not quite satisfactory when using small numbers of modes), and that it can be used to go beyond Hertz' theory. The functional dependence of the contact duration on the impact velocity, $\tau \propto v^{-1 / 5}$, has been verified in the simulation.

It has been shown that a small but noticeable amount of translational energy is converted into vibrations even at relatively small impact velocities of the order of $1 / 10$ of the transversal sound velocity, and that this amount is likely to increase if the accuracy, i.e. the number of modes, is increased.

The elastic deformation of the spheres does not lead to an effective friction mechanism upon grazing collisions, at least not up to impact velocities of $v=0.1$. This simplifies the discussion of the coefficients of restitution since only normal restitution is present. The coefficient of normal restitution is found to depend on the impact parameter in a non-trivial way, having a maximum at intermediate impact parameters.

The collision of spheres of unequal size shows strong deviations from the quasistatic case, namely a highly unsymmetric collision, a vibrational kinetic energy which is at times considerably higher than the potential energy, and a decreased coefficient of restitution as compared to a collision of equal spheres with the same impact velocity. This indicates that the energy loss mechanism through excitation of vibrations by collisions is particularly important for spheres which differ in size. 


\section{Conclusions and outlook}

\subsection{Conclusions}

The analysis of a two particle system with internal, vibrational degrees of freedom in Ch. 2 resulted in an explicit solution of the equations of motion which allowed for a detailed investigation into the properties of the probability density for the coefficient of restitution in two-particle collisions with unspecified ("thermal") initial conditions. Though no exact analytical expression could be found, an approximation was constructed which fulfilled all of the known exact properties of the probability density and which could very well be fitted to results from Monte Carlo simulations. Using this analytic, albeit approximate, probability density, many particle simulations were performed in $\mathrm{Ch}$. 3. As a result, it was shown that the system does not run into an inelastic collapse, so that the method is well suited to long simulation runs. Complex cluster dynamics take place during the approach to equilibrium in a system with total energy conservation (the equilibrium being a state where the total energy is uniformly distributed among all degrees of freedom, translational and vibrational). The equilibrium state itself was characterised by its dynamic structure factor and complete agreement with hydrodynamical calculations was observed, somewhat surprising for a one-dimensional system but explained by the stochastic nature of the collision which destroys memory and asserts the molecular-chaos assumption which is a central prerequisite for hydrodynamic theories. The approach to equilibrium is dominated by the slowest decaying mode which is the one with the largest wavelength. Finally, a system with damped vibrations was simulated which showed that the final state of such a system without total energy conservation is one where basically all particles are clumped together and all kinetic energy is lost. The dynamics of this system consisted of a formation of clusters in the initial stage, followed by "coarsening" in the sense that colliding clusters interacted completely inelastically, thus forming one larger cluster, until only one big cluster was left. The energy decay could of course not be described by anything like Haff's law [Haf83] but instead followed a succession of steps, each corresponding to a cluster collision.

Starting from the one-dimensional model including only a finite but arbitrary number of vibrational modes, a solution of the equations of motion in the hard-core limit was given by two methods: The first involved the explicit solution of a non- 
linear integral equation, the second arrived at the same result using simple energy conservation arguments. While the first is more convincing from a fundamental point of view, the latter is much simpler and could also be generalised to two and three dimensions. This generalisation allowed for a relatively efficient numerical simulation of a complete collision process of two elastic spheres. The simulations showed that the production of vibrations for colliding identical spheres is weak but noticeable for impact velocities around 1/10 of the transversal sound velocity. Comparison with Hertz' theory showed qualitative agreement for almost quasistatic collisions. Quantitative agreement could not be achieved due to computational limitations on the number of vibrational modes used. Collisions of spheres of unequal size but with the same impact velocity as before, however, are qualitatively different: The quasistatic assumption breaks down, collisions are unsymmetric in time, and excitation of vibrations becomes important. Thus one central question that was posed in the introduction, namely if Rayleigh's estimate [Ray06] of the relative unimportance of vibrations remains valid for the case of unequal spheres, can be answered negatively.

\subsection{Outlook}

While the one-dimensional system may not be of chief experimental relevance, it provides a simple testing ground for various theories. Since the cluster geometry as seen in the simulations is naturally simple in one dimension, it might be possible to make progress by trying to analyse the coarsening of clusters as it was described above. Thus one might arrive at a theory describing the energy decay in a situation which is far away from the homogeneous cooling state for which Haff's law applies (see also [BE:98a, vNF.99]). Other possible extensions of the one-dimensional systems are investigations of particles with a different set of vibrational modes; this is made possible (at least numerically) by the general solution of $\mathrm{Ch}$. 1 . One could for instance consider particles which do not only vibrate longitudinally but which also have transversal vibrations which could become weakly excited upon slightly non-central collisions. Numerous other variations are conceivable, e.g. particles consisting of a small number of "atoms" connected by springs, or particles containing defects. The main question would be if the modified microscopic details result in different macroscopic behaviour or if the macroscopic system is independent of such modifications and is universal in this respect. The same question could be addressed by using different expressions for the probability density $p_{\beta}(\epsilon)$ than the one given here.

The general method for collisions of three-dimensional objects being set up, one could ask a large number of further questions; simulations could be done for all conceivable values of the parameters. However, there are also some more fundamental questions that one might attempt to answer:

- It might be possible to solve the collision process of two spheres under special conditions exactly, using only a limited set of vibrational modes. An example 
where this is possible under certain conditions was given in Sec. 4.7. There it was argued that for slow velocities two colliding spheres can be regarded as if they were in a (half-sided) harmonic potential. Connected with this is the question if it is possible to carry out the limit of infinitely many modes in order to obtain a closed expression (but containing memory terms) for the three-dimensional system like Eq. (2.18) for the one-dimensional case. While such an expression would most likely still be unsolvable in practice (just as Eq. (2.18)), perhaps one could derive some exact results from it.

- In experiments it is found that elastic vibrations of solids are always damped by internal friction through various different mechanisms (see e.g. [Kol6.3], Ch. 5). These effects could be incorporated into the theory and simulations and thus one could try to reach quantitative agreement with experiments and other theories of viscoelastic impact [Pao55, KK87, HSB95, BSHP96]. This amounts to combining two of the three loss mechanisms (plastic deformation and fracture, viscoelastic behaviour, and elastic vibrations) into one general framework.

- Simulations of many particle systems using stochastic coefficients of restitution (normal and tangential) based on the model presented here can be performed. It is expected that they do not show inelastic collapse (for the same reason that it doesn't appear in one dimension) which would make them good candidates for long runs into the clustering regime, while at the same time being based on a microscopic model. Comparisons of this method with other methods (e.g. the TC model from [LM.98] or the rotation of rebound velocities by a small random angle as proposed in [DB.97]) could help decide whether such differences are important for macroscopic properties. 


\section{A. Details concerning the probability distribution $p_{\beta}(\epsilon)$}

\section{A.1. Implications of the non-negativity of the probability density}

In Sec. 2.3.1 use was made of a real, symmetric function $c(x, y)$ which was written as

$$
c(x, y)=\frac{1}{2} e^{-x-y-f(x, y)}
$$

with a real, symmetric function $f(x, y)$. This is a priori not the most general ansatz and may even lead to negative valued $p(x, y)$.

Hence we will argue in App. A.1.1 that a $c(x, y)$ that has negative values generates a negative-valued $p(x, y)$ and is thus unsuited. One can therefore restrict oneself to non-negative functions $c(x, y)$, which in turn can be written as in Eq. (A.1). The additional properties of $c(x, y)$, Eq. (2.48), are satisfied if $f(0, y)=y$ and $\lim _{x \rightarrow \infty}(x+$ $f(x, y))=\infty$. In App. A.1.2 it is shown that further restrictions on $f(x, y)$ follow from the non-negativity of $p(x, y)$.

\section{A.1.1. Proof that $c(x, y)$ is non-negative}

Suppose that $c(x, y)$ has negative values, i.e. $c\left(x_{1}, y_{1}\right)<0$ for some $x_{1}, y_{1}$. Since $c(\infty, y)=0$, there exists an $x_{2}>x_{1}$ such that $\partial c / \partial x\left(x_{2}, y_{1}\right)>0$. For every rational $\gamma=p / s$ we must have $c(x, y)=e^{-2 y} / 2$ for all $y>\xi^{-2} x$, where $\xi$ is defined in Eq. (2.35) (by the same argument that was used for Eq. (2.53)). This implies $\partial c / \partial x\left(x_{2}, y_{2}\right)=0$ for any $y_{2}>x_{2} \xi^{-2}$. But we already had that $\partial c / \partial x\left(x_{2}, y_{1}\right)>0$, from which we can see that $y_{1}<y_{2}$ and therefore there must exist a $y_{0} \in\left(y_{1}, y_{2}\right)$ where $\partial^{2} c /(\partial x \partial y)\left(x_{2}, y_{0}\right)<0$. Eq. (2.47) shows that this is equivalent to a negative value of the probability density: $p\left(x_{2}, y_{0}\right)<0$. 


\section{A.1.2. Proof that $\partial f / \partial x \geq-1$ and $\partial f / \partial y \geq-1$}

It is possible to deduce some restrictions on $f(x, y)$ from the non-negativity of $p(x, y)$. Suppose there were some $x_{0}, y_{0}$, such that

$$
\frac{\partial f}{\partial x}\left(x_{0}, y_{0}\right)<-1
$$

It follows from Eq. (2.53) that for any $\tilde{y}>\xi^{-2} x_{0}, \partial f / \partial x\left(x_{0}, \tilde{y}\right)=-1$. Thus there exists a $y^{*} \in\left(y_{0}, \xi^{-2} x_{0}\right]$ such that for any $y$ from a small neighbourhood of $y^{*}$

$$
\frac{\partial f}{\partial x}\left(x_{0}, y\right)<-1 \text { for } y<y^{*}
$$

and

$$
\frac{\partial f}{\partial x}\left(x_{0}, y\right) \geq-1 \text { for } y>y^{*} .
$$

holds. If we suppose that the leading non-constant term of $\partial f / \partial x\left(x_{0}, y\right)$ as $y$ tends to $y^{*}$ from below is of the order $n \geq 1$, i.e.

$$
\frac{\partial f}{\partial x}\left(x_{0}, y\right)=-1-\alpha\left(y^{*}-y\right)^{n}+\mathcal{O}\left(\left(y^{*}-y\right)^{n+1}\right) \quad \text { for } y<y^{*}
$$

$\alpha>0$ being the coefficient, then we have

$$
\frac{\partial^{2} f}{\partial x \partial y}\left(x_{0}, y\right)=\alpha n\left(y^{*}-y\right)^{n-1}+\mathcal{O}\left(\left(y^{*}-y\right)^{n}\right) .
$$

Here we see that at least in a small interval left of $y^{*}, \partial^{2} f /(\partial x \partial y)\left(x_{0}, y\right)>0$. (By inversion of this argument we can conclude that $\partial^{2} f /(\partial x \partial y)(x, y) \leq 0$ for all $x, y$ implies $\partial f / \partial x\left(x, y_{0}\right) \geq-1$ for all $x, y$. This will be needed below.) Plugging this into Eq. (2.52) we get

$$
\begin{aligned}
p\left(x_{0}, y\right)= & \frac{e^{y-x_{0}-f\left(x_{0}, y\right)}}{2}\left(\left(-\alpha\left(y^{*}-y\right)^{n}+\mathcal{O}\left(\left(y^{*}-y\right)^{n+1}\right)\right)\left(1+\frac{\partial f}{\partial y}\left(x_{0}, y\right)\right)-\right. \\
& \left.\left(\alpha n\left(y^{*}-y\right)^{n-1}+\mathcal{O}\left(\left(y^{*}-y\right)^{n}\right)\right)\right) \\
= & \frac{e^{y-x_{0}-f\left(x_{0}, y\right)}}{2}\left(-\alpha\left(y^{*}-y\right)^{n-1}\right)\left(\mathcal{O}\left(y^{*}-y\right)\left(1+\frac{\partial f}{\partial y}\left(x_{0}, y\right)\right)+n\right),(\mathrm{A} .7)
\end{aligned}
$$

which is bound to become negative in a small interval left of $y^{*}$. So unless $\partial f / \partial x\left(x_{0}, y\right) \rightarrow$ -1 faster than any power of $y^{*}-y$ as $y \rightarrow y^{*}$ (a case which we will ignore), the assumption Eq. (A.2) will lead to a non-acceptable $p(x, y)$. 
Therefore, we must restrict ourselves to those $f(x, y)$ which satisfy $\partial f / \partial x(x, y) \geq$ -1 for all $x, y$. We will, however, be even a bit more restrictive and demand that $\partial^{2} f /(\partial x \partial y)(x, y) \leq 0$ for all $x, y$. As we have seen above, this implies $\partial f / \partial x(x, y) \geq$ -1 and because of the symmetry of $f(x, y)$ we also have $\partial f / \partial y(x, y) \geq-1$. Thus all terms on the rhs of Eq. (2.52) are non-negative so this restriction guarantees a nonnegative $p(x, y)$. By a similar argument as above it can be seen that this condition also implies the additional property $\partial f / \partial x(x, y) \leq 1$ and $\partial f / \partial y(x, y) \leq 1$ for all $x, y$.

Thus we have proved that a function $f(x, y)$ that satisfies $\partial^{2} f /(\partial x \partial y) \leq 0$ not only generates a non-negative probability distribution but also has the useful properties $|\partial f / \partial x| \leq 1$ and $|\partial f / \partial y| \leq 1$.

\section{A.2. Evaluation of the low temperature limit}

In order to motivate Eq. (2.60), let's evaluate Eq. (2.54) a little further:

$$
\begin{aligned}
\frac{\langle x\rangle}{y}= & \frac{e^{y}}{2 y} \int_{0}^{\infty}\left(1+\frac{\partial f}{\partial y}(z y, y)\right) e^{-z y-f(z y, y)} y d z \quad \text { with } z=\frac{x}{y} \\
= & \frac{1}{2}\left(\int_{0}^{1}\left(1+\frac{\partial f}{\partial y}(z y, y)\right) e^{y(1-z)-f(z y, y)} d z+\right. \\
& \underbrace{\int_{1}^{\infty} \underbrace{\left(1+\frac{\partial f}{\partial y}(z y, y)\right)}_{\geq 0} e^{y(1-z)-f(z y, y)} d z)}_{\longrightarrow 0 \text { as } y \rightarrow \infty}
\end{aligned}
$$

The estimate for the bracket in the second integral in Eq. (A.8) follows from $|\partial f / \partial y| \leq 1$ which implies $0 \leq 1+\partial f / \partial y(z y, y) \leq 2$.

The limit of the second integral as $y \rightarrow \infty$ follows from $|\partial f / \partial x| \leq 1$ and $f(x, y)=$ $x-y$ for $x>\xi^{-2} y$ because these two conditions together ensure that $f(x, y) \geq x-y$ for all $x$ (see Fig. A.1). Thus the integral is bounded from above by $2 \int_{1}^{\infty} \exp (2 y(1-$ $z)) d z=1 / 2 y \rightarrow 0$.

In order to evaluate the first integral in Eq. A.8, we first note that $f(z y, y)=$ $y(1-z)$ for $z \leq \xi^{2}$ and $f(z y, y) \geq y(1-z)$ for $z>\xi^{2}$, which implies $\lim _{y \rightarrow \infty} y(1-$ $z)-f(z y, y)=0$ for $z \leq \xi^{2}$ and $\lim _{y \rightarrow \infty} y(1-z)-f(z y, y) \leq 0$ for $z>\xi^{2}$. Now suppose that there is some $\zeta \in\left(\xi^{2}, 1\right)$ such that

$$
\lim _{y \rightarrow \infty} y(1-z)-f(z y, y)= \begin{cases}0 & \text { if } 0 \leq z<\zeta \\ -\infty & \text { if } \zeta<z \leq 1\end{cases}
$$

Provided the convergence is fast enough, we can evaluate the first integral in Eq. A.8 as $y \rightarrow \infty$ by interchanging the limit with the integral and with $\frac{\partial}{\partial y}$ (note that 


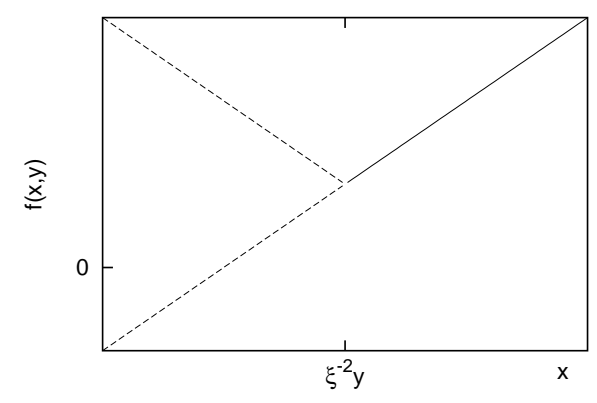

Figure A.1.: Sketch of the possible range of $f(x, y)$ as a function of $x$ for fixed $y$. $f(x, y)=x-y$ for $x>\xi^{-2} y$ and due to $|\partial f / \partial x| \leq 1, f(x, y)$ can only reside in the region between the dashed lines for $x<\xi^{-2} y$. Thus it follows that $f(x, y) \geq x-y$ for all $x, y$.

$\frac{\partial y(1-z)}{\partial y}=1$ because $\left.z=x / y\right)$ :

$$
\begin{aligned}
\lim _{y \rightarrow \infty} \frac{\langle x\rangle}{y} & =\lim _{y \rightarrow \infty} \frac{1}{2} \int_{0}^{1}\left(1+\frac{\partial f}{\partial y}(z y, y)\right) e^{y(1-z)-f(z y, y)} d z \\
& =\frac{1}{2} \int_{0}^{1} \lim _{y \rightarrow \infty}\left(1+\frac{\partial f}{\partial y}(z y, y)\right) e^{y(1-z)-f(z y, y)} d z \\
& =\frac{1}{2} \int_{0}^{\zeta} 2 d z=\zeta .
\end{aligned}
$$

Additionally, we can compute $\frac{\langle\sqrt{x}\rangle}{\sqrt{y}}$ according to Eq. 2.5.5 in the same way, which gives

$$
\lim _{y \rightarrow \infty} \frac{\langle\sqrt{x}\rangle}{\sqrt{y}}=\sqrt{\zeta}
$$

In other words: A sufficient condition for the low temperature limit, Eq. (2.41), is just Eq. (A.9) with $\zeta=\gamma^{2}$.

\section{A.3. Evaluation of the high temperature limit}

In the high-temperature limit, the right hand side of Eq. (2.63) must be a constant. From this and the symmetry condition for $\tilde{f}(z, y)$ we can conclude that

$$
\tilde{f}(z, 0)=c_{1} \sqrt{z}+c_{2}(z+1)
$$

with some constants $c_{1}$ and $c_{2}$. We know that outside this range for $\epsilon$, when $z \leq \xi^{2}$ or $z \geq \xi^{-2}, \tilde{f}(z, 0)=|1-z|$. $\tilde{f}(z, y)$ must be continuously differentiable with respect 
to $z$ at least once, otherwise $p_{\beta}(\epsilon)$ would have a $\delta$-peak due to the $\frac{\partial^{2} \tilde{f}}{\partial z^{2}}$ term that appears in Eq. (2.62).

Thus we have the requirements that at the endpoint of the range, i.e. at $z=\xi^{2}$,

$$
\begin{aligned}
1-\xi^{2} & =c_{1} \xi+c_{2}\left(\xi^{2}+1\right) \quad \text { and } \\
\left.\frac{\partial(1-z)}{\partial z}\right|_{z=\xi^{2}}=-1 & =\left.\frac{\partial}{\partial z}\left(c_{1} \sqrt{z}+c_{2}(z+1)\right)\right|_{z=\xi^{2}}=\frac{c_{1}}{2 \xi}+c_{2} .
\end{aligned}
$$

These two equations fix $c_{1}$ and $c_{2}$ :

$$
\begin{aligned}
c_{1} & =-\frac{4 \xi}{1-\xi^{2}} \\
c_{2} & =\frac{1+\xi^{2}}{1-\xi^{2}} .
\end{aligned}
$$

$\tilde{f}(z, 0)$ is thus completely determinded by the high temperature limit:

$$
\tilde{f}(z, 0)= \begin{cases}1-z & \text { for } z \leq \xi^{2} \\ -\frac{4 \xi}{1-\xi^{2}} \sqrt{z}+\frac{1+\xi^{2}}{1-\xi^{2}}(z+1) & \text { for } \xi^{2}<z<\xi^{-2} \\ z-1 & \text { for } z \geq \xi^{-2}\end{cases}
$$

\section{A.4. "Proof" for a uniform distribution at $\beta=0$}

As indicated by the quotation marks in the heading, we will only outline the basic ideas of the proof for the sake of shortness.

We start from Eq. 2.18 and take some realization of the stochastic process $q(\tau)$. Then we vary the amplitude of this realization by multiplying it with some $\alpha$, which we let tend to $\infty$. This is in accordance with Eq. (2.21), which states that the covariance of $q(\tau)$ goes to infinity as $\beta$ goes to 0 . We also have to scale $\tau_{0}$ with $\alpha$ in order to assure that $\tau-\alpha \tau_{0}+\alpha q(\tau) \leq 0$ for all $\tau<0$. This is simply the condition that at $\tau=0$ the particles are still well separated and haven't touched before in this collision event.

Consider now a rescaled version of eq. (2.18):

$$
\begin{aligned}
w^{\prime}(\tau) & =\max (0, f(\tau)), \quad \text { where } \\
f(\tau) & =\max _{\tau^{\prime} \in[0, \tau]}\left\{\frac{\tau^{\prime}}{\alpha}-\tau_{0}-\sum_{i, \nu} w^{\prime}\left(\tau^{\prime}-\nu \Gamma_{i}\right)+q\left(\tau^{\prime}\right)\right\}
\end{aligned}
$$

with $w^{\prime}(\tau)=w(\tau) / \alpha$. When $\gamma$ is a rational number, $\gamma=p / s$, we know that $q(\tau)$ is periodic with period $p+s$ (see Sec. 2.2.3). Hence there exists a $\tilde{\tau}$ in the period from 0 to $p+s$ where $q(\tau)$ takes on its maximum value. This will be the only point in this period where $q(\tau)$ assumes this value because the probability for a degenerate maximum is vanishingly small. 
When $\alpha \rightarrow \infty$, it is clear that the smallest $\tau^{*}$ where $\frac{\tau^{*}}{\alpha}-\tau_{0}+q\left(\tau^{*}\right)$ crosses the axis (this marks the beginning of the collision) is very close to the position of the maximum of $q(\tau)$ in a particular period, i.e. $\tau^{*} \approx \tilde{\tau}+n(p+s)$ with some $n \in \mathbb{N}$. Also, provided that $\alpha$ is large enough, the next time when the axis is crossed from below will be approximately one period later, namely at around $\tilde{\tau}+(n+1)(p+s)$. Therefore we see that $w^{\prime}(\tau)$ reaches a plateau very shortly after the beginning of the collision, the height of the plateau being given approximately by $z=(\tilde{\tau}+n(p+s)) / \alpha-\tau_{0}+q(\tilde{\tau})$. This height can be varied continuously from 0 to $(p+s) / \alpha$ by using a different realisation of the stochastic process (but with the same statistical weight), namely $q^{\prime}(\tau)=q(\tau+\Delta \tau)$. This is because $q^{\prime}(\tau)$ has a different position of the maximum, $\tilde{\tau}^{\prime}=\tilde{\tau}-\Delta \tau \bmod p+s$, possibly a different period $n^{\prime}=n-1, n$ or $n+1$ of the beginning of the collision, $\tau^{*} \approx \tilde{\tau}^{\prime}+n^{\prime}(p+s)$ and it thus leads to the plateau height $z=\left(\tilde{\tau}^{\prime}+n^{\prime}(p+s)\right) / \alpha-\tau_{0}+q^{\prime}\left(\tilde{\tau}^{\prime}\right)=(\tilde{\tau}-\Delta \tau+n(p+s)) / \alpha-\tau_{0}+q(\tilde{\tau}) \bmod (p+s) / \alpha$.

Since this is true for any realisation of $q(\tau)$, we can conclude that the height $z$ of the initial plateau is uniformly distributed between 0 and $(p+s) / \alpha$.

We have just seen that $\frac{\tau}{\alpha}-\tau_{0}+q(\tau)<0$ for $\tilde{\tau}+n(p+s)+\delta<\tau<\tau^{*}+p+s-\delta^{\prime}$ with some small $\delta, \delta^{\prime}>0$. Therefore any additional contributions (if any) to $w^{\prime}(\tau)$ must come from times $\tau^{*}+p+s-\delta^{\prime}<\tau<\tau^{*}+p+s$ (remember the maximum collision time is $p+s$ ). When we let $\tau \nearrow \tau^{*}+p+s$, we can evaluate Eq. (A.16) because then all the $p+s-2$ non-zero memory terms are equal to $z$. Thus we get:

$$
w^{\prime}\left(\tau^{*}+p+s\right)=\max \left(z, \frac{p+s}{\alpha}-(p+s-2) z\right) .
$$

Here, we must distinguish two cases: $z<\frac{1+\xi}{\alpha}$ and $z>\frac{1+\xi}{\alpha}(\xi$ was defined in Eq. (2.35)). In the former case, the first argument of the max-function in Eq. (A.17) is smaller than the second, in the latter case it is the second argument that is smaller than the first. This means that for $z>\frac{1+\xi}{\alpha}, \epsilon+1=w\left(\tau^{*}+p+s\right)=\alpha w^{\prime}\left(\tau^{*}+p+s\right)=$ $\alpha z \in(1+\xi, p+s]$ and for $z<\frac{1+\xi}{\alpha}, \epsilon+1=p+s-(p+s-2) \alpha z \in(1+\xi, p+s]$. Since $z$ is uniformly distributed over its range, we conclude that $\epsilon$ is also uniformly distributed between $1 /(p+s-1)$ and $p+s-1$. 


\section{B. On the hard-core limit of the generalised one-dimensional collision equation}

In the first part of this appendix, Eq. (2.12) is solved in the hard-core limit $\alpha \rightarrow \infty$. Some calculational details are postponed to Apps. B.2 and B.3.

\section{B.1. Solution of the equation of motion (2.12) in the hard-core limit}

Without loss of generality it can be assumed that the time of first contact (in the true hard-core limit, or, equivalently, if the potential were switched off) is at $t=0$. In this limit, the end-to-end distance $r(t)=R\left(T_{0}\right)+\left(t-T_{0}\right) \dot{R}\left(T_{0}\right)+(\tilde{G} * \ddot{R})(t)+Q(t)$ thus equals 0 for $t=0$ (as in Sec. 2.1.1, the convolution integral extends over the interval from $T_{0}<0$ to $t$ ). If the potential were zero, the acceleration $\ddot{R}(t)$ would be zero for all $t$, thus the convolution integral would vanish and the end-to-end distance would be given by $R\left(T_{0}\right)+\left(t-T_{0}\right) \dot{R}\left(T_{0}\right)+Q(t)$ only. Thus choosing the time of first contact to be at $t=0$ amounts to requiring $R\left(T_{0}\right)+\left(t-T_{0}\right) \dot{R}\left(T_{0}\right)+Q(t)=0$ for $t=0$ and $R\left(T_{0}\right)+\left(t-T_{0}\right) \dot{R}\left(T_{0}\right)+Q(t)>0$ for $T_{0} \leq t<0$. The expression $R\left(T_{0}\right)+\left(t-T_{0}\right) \dot{R}\left(T_{0}\right)+Q(t)$ can be split in two parts, the linear approach to zero around $t=0$ and the rest (denoted by $\phi(t)$ ),

$$
R\left(T_{0}\right)+\left(t-T_{0}\right) \dot{R}\left(T_{0}\right)+Q(t)=-v t+\phi(t) .
$$

Here, $v=-\left.\frac{d}{d t}\left(R\left(T_{0}\right)+\left(t-T_{0}\right) \dot{R}\left(T_{0}\right)+Q(t)\right)\right|_{t=0}>0$. With this definition of $v$, $\phi(t)$ obeys $\phi(0)=\dot{\phi}(0)=0$.

Eq. (2.12) can now be rewritten as

$$
\ddot{R}(t)=\frac{\alpha B}{\mu} e^{-\alpha(-v t+\phi(t)+(\tilde{G} * \ddot{R})(t))} .
$$

Eq. (B.2) can be solved exactly under the condition that $\phi(t) \equiv 0$ and $\tilde{G}(t)=$ $t \gamma \Theta(t)$. The latter condition amounts to approximating $\tilde{G}(t)=t \gamma \Theta(t)+G_{1}(t)$ by its 
initial slope $\gamma=\tilde{G}^{\prime}\left(0^{+}\right)$, i.e. setting $G_{1} \equiv 0$. In this approximation, Eq. (B.2) reads (renaming $\ddot{R}$ to $\ddot{X}_{\alpha}$ for later reference)

$$
\ddot{X}_{\alpha}(t)=\frac{\alpha B}{\mu} \exp \left(-\alpha\left(-v t+\gamma \int_{T_{0}}^{t} d \tau(t-\tau) \ddot{X}_{\alpha}(\tau)\right)\right) .
$$

It can be checked by a simple integration that

$$
\ddot{X}_{\alpha}(t)=\frac{\alpha v_{\alpha}^{2}}{2 \gamma} \cosh ^{-2}\left(\frac{\alpha v_{\alpha}}{2} t+\eta_{\alpha}\right)
$$

with $v_{\alpha}=\sqrt{v^{2}+2 B \gamma e^{\alpha v T_{0}} / \mu}$ and $\eta_{\alpha}=-\alpha v_{\alpha} T_{0} / 2-\operatorname{artanh}\left(v / v_{\alpha}\right)$ is indeed a solution of Eq. (B.3). It is easy to see that $v_{\alpha} \rightarrow v$ and $\eta_{\alpha} \rightarrow \eta=\ln \left(B \gamma / 2 \mu v^{2}\right)$ exponentially fast as $\alpha \rightarrow \infty$.

Returning to the full equation $(\overline{B .2})$, one can write, without loss of generality, the acceleration $\ddot{R}$ in terms of the solution $\ddot{X}_{\alpha}$ from Eq. (B.4) and an unknown function $f_{\alpha}(t)$ as

$$
\ddot{R}(t)=\ddot{X}_{\alpha}(t) e^{-\alpha\left(\phi(t)+\left(G_{1} * \ddot{X}_{\alpha}\right)(t)\right.} e^{f_{\alpha}(t)}
$$

and insert this ansatz into Eq. (B.2). Thus one obtains an equation for the newly introduced function $f_{\alpha}(t)$. After a few manipulations, the result is given by

$$
f_{\alpha}(t)=-\alpha\left[\tilde{G} *\left(\ddot{X}_{\alpha}\left(e^{-\alpha F_{\alpha}+f_{\alpha}}-1\right)\right)\right](t)
$$

with the abbreviation $F_{\alpha}(t)=\phi(t)+\left(G_{1} * \ddot{X}_{\alpha}\right)(t)$. The function $F_{\alpha}$ has the following properties (see App. B.3):

$$
\begin{aligned}
\lim _{\alpha \rightarrow \infty} \alpha^{3} F_{\alpha}(0) & =C_{1} \\
\lim _{\alpha \rightarrow \infty} \alpha^{2} \dot{F}_{\alpha}(0) & =C_{2} \\
\lim _{\alpha \rightarrow \infty} \ddot{F}_{\alpha}(0) & =\ddot{\phi}(0) \\
\left|F_{\alpha}^{(3)}(x)\right| & <C_{3}(K) \quad \text { for all } \alpha \text { and } x \in K,
\end{aligned}
$$

with certain constants $\left|C_{1}\right|,\left|C_{2}\right|, C_{3}(K)<\infty$ and any compact subset $K \subset \mathbb{R}$.

If one should find that $f_{\alpha}$ converges to a continuous function $f_{\infty}$ as $\alpha \rightarrow \infty$, the task of finding the hard-core limit of Eq. (B.2) is solved: Since $v_{\alpha} \rightarrow v, \eta_{\alpha} \rightarrow \eta$, it is easily seen that $\ddot{X}_{\alpha} \rightarrow 2 v \delta(t) / \gamma$. The properties given in Eqs. (B.7) ensure that the term $\alpha F_{\alpha}(t)$ is regular enough in order not to spoil the convergence of $\ddot{R}(t)=\ddot{X}_{\alpha}(t) e^{-\alpha F_{\alpha}(t)+f_{\alpha}(t)}$ to a $\delta$-function, hence it follows that $\ddot{R}(t)$ converges to $2 v e^{f_{\infty}(0)} \delta(t) / \gamma$. 
Therefore I will proceed to argue that $f_{\infty}$ exists, that it is continuous and that $f_{\infty}(0)=0$. To this end, a change of variables $\xi=\alpha \tau$ is performed in the convolution integral in Eq. (B.6) and the exponential is expanded:

$$
\begin{aligned}
f_{\alpha}(t) & =-\frac{\alpha v_{\alpha}^{2}}{2 \gamma} \int_{\alpha T_{0}}^{\alpha t} d \xi \tilde{G}\left(t-\frac{\xi}{\alpha}\right) \cosh ^{-2}\left(\frac{v_{\alpha} \xi}{2}+\eta_{\alpha}\right) \sum_{k=1}^{\infty} \frac{\left(f_{\alpha}\left(\frac{\xi}{\alpha}\right)-\alpha F_{\alpha}\left(\frac{\xi}{\alpha}\right)\right)^{k}}{k !} \\
& =-\frac{v_{\alpha}^{2}}{2 \gamma} \int_{\alpha T_{0}}^{\alpha t} d \xi \tilde{G}\left(t-\frac{\xi}{\alpha}\right) \cosh ^{-2}\left(\frac{v_{\alpha} \xi}{2}+\eta_{\alpha}\right) \sum_{k=1}^{\infty} \frac{\left(\alpha f_{\alpha}\left(\frac{\xi}{\alpha}\right)-\alpha^{2} F_{\alpha}\left(\frac{\xi}{\alpha}\right)\right)^{k}}{\alpha^{k-1} k !}
\end{aligned}
$$

By introducing the function $\tilde{f}_{\alpha}(\zeta):=\alpha f\left(\frac{\zeta}{\alpha}\right)$, this can be written as

$$
\tilde{f}_{\alpha}(\zeta)=-\frac{\alpha v_{\alpha}^{2}}{2 \gamma} \int_{\alpha T_{0}}^{\zeta} d \xi \tilde{G}\left(\frac{\zeta-\xi}{\alpha}\right) \cosh ^{-2}\left(\frac{v_{\alpha} \xi}{2}+\eta_{\alpha}\right) \sum_{k=1}^{\infty} \frac{\left(\tilde{f}_{\alpha}(\xi)-\alpha^{2} F_{\alpha}\left(\frac{\xi}{\alpha}\right)\right)^{k}}{\alpha^{k-1} k !}
$$

This equation now allows an expansion in $1 / \alpha$. By Taylor expanding $\tilde{G}(t)=\bar{G}(t) \Theta(t)$ (where $\bar{G}(t)$ is the analytic continuation of $\tilde{G}(t))$ and $\alpha^{2} F_{\alpha}(\xi / \alpha)$ one obtains

$$
\begin{aligned}
\tilde{f}_{\alpha}(\zeta)=-\frac{\alpha v_{\alpha}^{2}}{2 \gamma} & \int_{\alpha T_{0}}^{\zeta} d \xi \sum_{n=0}^{\infty} \frac{\bar{G}^{(n)}(0)}{n !} \frac{(\zeta-\xi)^{n}}{\alpha^{n}} \cosh ^{-2}\left(\frac{v_{\alpha} \xi}{2}+\eta_{\alpha}\right) \\
& \times \sum_{k=1}^{\infty} \frac{\left(\tilde{f}_{\alpha}(\xi)-\alpha^{2} F_{\alpha}(0)-\alpha^{2} \dot{F}_{\alpha}(0) \frac{\xi}{\alpha}-\frac{\alpha^{2} \ddot{F}_{\alpha}(0)}{2}\left(\frac{\xi}{\alpha}\right)^{2}-\cdots\right)^{k}}{\alpha^{k-1} k !}
\end{aligned}
$$

Exploiting again the properties of $F_{\alpha}$ from Eqs. (B.7) and keeping only the zeroth order terms (the term proportional to $\alpha$ vanishes since $\bar{G}(0)=0$ ), one gets

$$
\begin{aligned}
\tilde{f}_{\alpha}(\zeta) & =-\frac{v^{2}}{2 \gamma} \int_{-\infty}^{\zeta} d \xi \bar{G}^{\prime}(0)(\zeta-\xi) \cosh ^{-2}\left(\frac{v \xi}{2}+\eta\right)\left(\tilde{f}_{\alpha}(\xi)-\frac{\ddot{\phi}(0)}{2} \xi^{2}\right)+\mathcal{O}\left(\frac{1}{\alpha}\right) \\
& =-\frac{v^{2}}{2} \int_{-\infty}^{\zeta} d \xi(\zeta-\xi) \cosh ^{-2}\left(\frac{v \xi}{2}+\eta\right)\left(\tilde{f}_{\alpha}(\xi)-\frac{\ddot{\phi}(0)}{2} \xi^{2}\right)+\mathcal{O}\left(\frac{1}{\alpha}\right)
\end{aligned}
$$


This is a linear integral equation which can be solved exactly. By differentiating Eq. (B.13) twice with respect to $\zeta$, it is transformed into an ordinary differential equation,

$$
\tilde{f}_{\infty}^{\prime \prime}(\zeta)=-\frac{v^{2}}{2} \cosh ^{-2}\left(\frac{v \zeta}{2}+\eta\right)\left(\tilde{f}_{\infty}(\zeta)-\frac{\ddot{\phi}(0)}{2} \zeta^{2}\right)
$$

Here, the index has been changed to $\infty$ since omitting the higher-order corrections in $1 / \alpha$ amounts to setting $\alpha=\infty$. The solution $\tilde{f}_{\infty}(\zeta)$ of this equation satisfying the boundary conditions $\lim _{\zeta \rightarrow-\infty} \tilde{f}_{\infty}(\zeta)=0$ and $\lim _{\zeta \rightarrow-\infty} \tilde{f}_{\infty}^{\prime}(\zeta)=0$ (which follow from Eq. $(\overline{B .10})$ ) can be found in App. B.2, Eqs. (B.29) and (B.30). Corrections to this solution are expected to be of order $\mathcal{O}(1 / \alpha)$.

The function $\tilde{f}_{\infty}$ itself does not contain enough information to reconstruct the original function $f_{\alpha}$ in the limit $\alpha \rightarrow \infty$ since the higher-order corrections may also become relevant in the limiting process. However, the missing information is contained in Eq. (B.9): In order to find $f_{\alpha}$, the solution for $\tilde{f}_{\alpha}=\tilde{f}_{\infty}+\mathcal{O}(1 / \alpha)$ can be inserted into the rhs of Eq. (B.9) instead of the term $\alpha f(\xi / \alpha)$. The integral that appears in the zeroth-order term of this equation can then be performed exactly, yielding $4 \tilde{G}(t) \ddot{\phi}(0)\left(\eta+\eta^{2}+\pi^{2} / 12\right) /(\gamma v)$. The final result for $f_{\alpha}$ is thus:

$$
f_{\alpha}(t)=\frac{4 \ddot{\phi}(0)}{\gamma v}\left(\eta+\eta^{2}+\frac{\pi^{2}}{12}\right) \tilde{G}(t)+\mathcal{O}\left(\frac{1}{\alpha}\right) .
$$

The limit $\alpha \rightarrow \infty$ is now simply

$$
f_{\infty}(t)=\frac{4 \ddot{\phi}(0)}{\gamma v}\left(\eta+\eta^{2}+\frac{\pi^{2}}{12}\right) \tilde{G}(t)
$$

Thus the limit exists, is continuous, and takes on the value 0 at $t=0$.

Returning to the original problem, this means that the acceleration $\ddot{R}$ is indeed given by

$$
\ddot{R}=\frac{2 v}{\gamma} \delta(t)
$$

in the hard-core limit. It must be mentioned that this result for $\ddot{R}$ is only valid in a time interval around $t=0$ that is small enough to exclude the (possibly existing) next contact of the particles. This next contact, however, can be treated by exactly the same method, yielding another $\delta$-function, and so on. Thus the whole collision process consists of a (finite) succession of small $\delta$-collisions. 


\section{B.2. Solution of the differential equation (B.14)}

Eq. (B.14) can be simplified by introducing a new function $g(\xi)$ defined by

$$
g(\xi)=\tilde{f}_{\infty}\left(\frac{2}{v}(\xi-\eta)\right) .
$$

This results in the following differential equation for $g(\xi)$,

$$
g^{\prime \prime}(\xi)=-2 \cosh ^{-2}(\xi)\left(g(\xi)-\frac{\ddot{\phi}(0)}{2}\left(\frac{2}{v}(\xi-\eta)\right)^{2}\right) \text {. }
$$

The corresponding homogeneous equation,

$$
g_{\text {hom }}^{\prime \prime}(\xi)+2 \cosh ^{-2}(\xi) g_{\text {hom }}(\xi)=0,
$$

can be transformed into a first order differential equation by the ansatz

$$
g_{\text {hom }}(\xi)=h(\xi) \tanh (\xi)
$$

which yields

$$
\tanh (\xi) h^{\prime \prime}(\xi)+2 \cosh ^{-2}(\xi) h^{\prime}(\xi)=0 \text {. }
$$

The general solution of this differential equation can be found by separation of the variables and is given by

$$
h^{\prime}(\xi)=c_{1} \tanh ^{-2}(\xi),
$$

where $c_{1}$ is an arbitrary constant. The function $g_{\mathrm{hom}}(\xi)$ can then be obtained by integrating $h^{\prime}(\xi)$ (and adding an arbitrary constant of integration $c_{2}$ ),

$$
\begin{aligned}
g_{\mathrm{hom}}(\xi) & =h(\xi) \tanh (\xi)=\left(\int d \xi c_{1} \tanh ^{-2}(\xi)+c_{2}\right) \tanh (\xi) \\
& =c_{1}(\xi \tanh (\xi)-1)+c_{2} \tanh (\xi)
\end{aligned}
$$

The inhomogeneous equation, Eq. (B.19), can now be solved by the method of variation of the constants, i.e. by assuming a $\xi$-dependence of $c_{1}$ and $c_{2}$. A straightforward calculation results in the following expression for a particular solution of Eq. (B.19), where

$$
I(\xi)=\frac{4 \ddot{\phi}(0)}{v^{2}} \cosh ^{-2}(\xi)(\xi-\eta)^{2}
$$


is the inhomogeneity:

$g_{\mathrm{part}}(\xi)=(\xi \tanh (\xi)-1) \int_{0}^{\xi} d \xi^{\prime} I\left(\xi^{\prime}\right) \tanh \left(\xi^{\prime}\right)-\tanh (\xi) \int_{0}^{\xi} d \xi^{\prime} I\left(\xi^{\prime}\right)\left(\xi^{\prime} \tanh \left(\xi^{\prime}\right)-1\right)$.

The integrals appearing in this equations can be solved exactly but are omitted here for the sake of shortness.

The solution satisfying the boundary conditions $g(-\infty)=g^{\prime}(-\infty)=0$ is given by the sum of the particular solution $g_{\text {part }}(\xi)$ and a suitable homogeneous solution, i.e. some $g_{\text {hom }}(\xi)$ from Eq. (B.24) with a suitable choice of the constants $c_{1}$ and $c_{2}$ (which really are constants for the homogeneous case). By evaluating the integrals in Eq. (B.27) and analysing the limit $\xi \rightarrow-\infty$ it is found that asymptotically

$$
g_{\text {part }}(\xi) \underset{\xi \rightarrow-\infty}{\rightsquigarrow}-\frac{4 \ddot{\phi}(0)}{v^{2}}\left(\ln 2+\eta+\frac{\eta^{2}}{2}\right) \xi-\frac{4 \ddot{\phi}(0)}{v^{2}}\left(\ln 2+\eta+\frac{\pi^{2}}{24}\right)+\mathcal{O}\left(\frac{1}{\xi}\right) .
$$

The homogeneous solution must cancel the two terms which do not tend to zero for $\xi \rightarrow-\infty$. This condition fixes the coefficients $c_{1}$ and $c_{2}$ for the homogeneous solution. It is easy to verify that $c_{1}=-4 \ddot{\phi}(0)\left(\ln 2+\eta+\eta^{2} / 2\right) / v^{2}$ and $c_{2}=2 \ddot{\phi}(0)\left(\eta^{2}-\pi^{2} / 12\right) / v^{2}$ do the job; thus the complete solution of Eq. (B.19) satisfying the appropriate boundary conditions is given by

$$
\begin{array}{r}
g(\xi)=\left(\int_{0}^{\xi} d \xi^{\prime} I\left(\xi^{\prime}\right) \tanh \left(\xi^{\prime}\right)-\frac{4 \ddot{\phi}(0)}{v^{2}}\left(\ln 2+\eta+\frac{\eta^{2}}{2}\right)\right)(\xi \tanh (\xi)-1)- \\
\left(\int_{0}^{\xi} d \xi^{\prime} I\left(\xi^{\prime}\right)\left(\xi^{\prime} \tanh \left(\xi^{\prime}\right)-1\right)-\frac{2 \ddot{\phi}(0)}{v^{2}}\left(\eta^{2}-\frac{\pi^{2}}{12}\right)\right) \tanh (\xi) .
\end{array}
$$

Finally, this result can be converted into the function $\tilde{f}_{\infty}(\zeta)$ by

$$
\tilde{f}_{\infty}(\zeta)=g\left(\frac{v \zeta}{2}+\eta\right)
$$

\section{B.3. Properties of the function $F_{\alpha}$}

The function $F_{\alpha}$ posseses some properties, summarised in Eqs. (B.7), which were needed in order to solve the equations of motion. In this appendix, these properties are going to be derived. The results of this appendix are due to [M̈̈199].

The function $F_{\alpha}$ is given by

$$
F_{\alpha}(t)=\phi(t)+\left(G_{1} * \ddot{X}_{\alpha}\right)(t),
$$


with $\phi(t), G_{1}(t)$, and $\ddot{X}_{\alpha}(t)$ as defined in App. B.1. In particular, the definition of $G_{1}$ implies that

$$
G_{1}(0)=\dot{G}_{1}(0)=\ddot{G}_{1}(0)=0
$$

and that $G_{1}^{(3)}(t)$ is bounded. Thus a Taylor expansion of $G_{1}(t)$ up to second order around $t=0$ only consists of the remainder term,

$$
G_{1}(t)=\frac{G_{1}^{(3)}\left(u_{t} t\right)}{3 !}
$$

where $u_{t}$ depends on $t$ and takes on some value between 0 and 1 . This implies, since $G_{1}^{(3)}(t)$ is bounded,

$$
\begin{aligned}
\alpha^{3} F_{\alpha}(0) & =\alpha^{3} \int_{T_{0}}^{0} d t^{\prime} G_{1}\left(-t^{\prime}\right) \ddot{X}_{\alpha}\left(t^{\prime}\right) \\
& =\int_{T_{0}}^{0} d t^{\prime} \frac{\alpha^{3}\left(-t^{\prime}\right)^{3}}{3 !} G_{1}^{(3)}\left(u_{t^{\prime}} t^{\prime}\right) \ddot{X}_{\alpha}\left(t^{\prime}\right) \\
& =-\frac{v_{\alpha}^{2}}{12 \gamma} \int_{\alpha T_{0}}^{0} d \xi \xi^{3} \cosh ^{-2}\left(\frac{v_{\alpha} \xi}{2}+\eta_{\alpha}\right) G_{1}^{(3)}\left(u_{\xi / \alpha} \frac{\xi}{\alpha}\right) \\
& \underset{\alpha \rightarrow \infty}{\longrightarrow}-\frac{v^{2} G_{1}^{(3)}(0)}{12 \gamma} \int_{-\infty}^{0} d \xi \xi^{3} \cosh ^{-2}\left(\frac{v \xi}{2}+\eta\right)=: C_{1} .
\end{aligned}
$$

The last two lines follow if $\ddot{X}_{\alpha}(t)$ from Eq. (B.4) is inserted.

A analogous calculation for $\dot{F}_{\alpha}$ yields

$$
\alpha^{2} \dot{F}_{\alpha}(0) \underset{\alpha \rightarrow \infty}{\longrightarrow} \frac{v^{2} G_{1}^{(3)}(0)}{4 \gamma} \int_{-\infty}^{0} d \xi \xi^{2} \cosh ^{-2}\left(\frac{v \xi}{2}+\eta\right)=: C_{2}
$$

By the same token the limit of the second derivative can be calculated, but since in this case the term $\ddot{\phi}(0)$, which is in general nonzero, becomes important, it follows that

$$
\ddot{F}_{\alpha}(0) \underset{\alpha \rightarrow \infty}{\longrightarrow} \ddot{\phi}(0)
$$

Finally, the third derivative is given by

$$
F_{\alpha}^{(3)}(t)=\phi^{(3)}(t)+\int_{T_{0}}^{0} d t^{\prime} G_{1}^{(3)}\left(t-t^{\prime}\right) \ddot{X}_{\alpha}\left(t^{\prime}\right)
$$

(since $G_{1}(0)=\dot{G}_{1}(0)=\ddot{G}_{1}(0)=0$, the third derivative of the convolution integral consists only of the one term shown). This expression converges for all $t$. Therefore, for all compact subsets $K \subset \mathbb{R}$, there exists a constant $C_{3}(K)<\infty$ such that $\left|F_{\alpha}^{(3)}(t)\right| \leq C_{3}(K)$ for all $t \in K . C_{3}(K)$ can be chosen to be independent of $\alpha$ since $G_{1}^{(3)}(t)$ is continuous and bounded and $\ddot{X}_{\alpha}(t)$ simply converges to a $\delta$-function. This concludes the derivation of the properties given in Eqs. (B.7). 


\section{Summary of Hertz' contact theory}

A detailed treatment of Hertz' quasistatic contact theory [Her82] can be found in standard textbooks, e.g. in [LL.91a] or [Gol60]. Here, only a short list of the essential results shall be given for reference.

Consider two spheres with radii $R_{1}$ and $R_{2}$, masses $M_{1}$ and $M_{2}$, transversal sound velocities $c_{\mathrm{t} 1}$ and $c_{\mathrm{t} 2}$, and Poisson numbers $\sigma_{1}$ and $\sigma_{2}$. As in Sec. 5.2, Eqs. (5.4) (5.6), the quantities $L, c$, and $M$ can be introduced as units of length, velocity, and mass, respectively. The "overlap" $\delta$ of the two spheres is defined as the distance by which their centres of mass are closer than the sum of their radii, and 0 if they are further apart than this, i.e.

$$
\delta=\max \left(0, R_{1}+R_{2}-\left|\boldsymbol{R}_{2}-\boldsymbol{R}_{1}\right|\right)
$$

In the quasistatic approximation, the contact face which exists when the spheres touch is perpendicular to the vector connecting the centres of mass for symmetry reasons. The force between the spheres thus acts in the same direction as this vector. The magnitude of the force is given by

$$
F=\left(\frac{\delta}{L}\right)^{3 / 2} \frac{L^{2}}{\sqrt{2} D},
$$

where $D$ is the following constant,

$$
D=\frac{\pi}{2}\left(\left(1-\sigma_{1}\right) \frac{R_{1}^{3}}{M_{1} c_{\mathrm{t} 1}}+\left(1-\sigma_{2}\right) \frac{R_{2}^{3}}{M_{2} c_{\mathrm{t} 2}}\right)
$$

For two identical spheres, this simplifies to $D=\pi(1-\sigma)$ in dimensionless units (i.e. $L=c=M=1)$.

Accordingly, the potential energy of the elastic deformation of the spheres reads

$$
E_{\mathrm{pot}}=\left(\frac{\delta}{L}\right)^{5 / 2} \frac{\sqrt{2} L^{3}}{5 D}
$$

since differentiating Eq. (C.4) with respect to $\boldsymbol{R}_{i}$ yields the force whose modulus is given in Eq. (C.2). 
The duration $\tau$ of a collision is given in terms of the impact velocity $v$ by

$$
\tau=\frac{4 \sqrt{\pi} \Gamma(2 / 5)}{5 \Gamma(9 / 10)}\left(\frac{25}{32} \cdot \frac{M^{2} D^{2}}{L c}\right)^{1 / 5}\left(\frac{v}{c}\right)^{-1 / 5},
$$

where $\Gamma(x)$ denotes the usual Gamma function. 


\section{Bibliography}

[AGZ98a] T. Aspelmeier, F. Gerl, and A. Zippelius. A microscopic model of energy dissipation in granular collisions. In H. J. Herrmann, J.-P. Hovi, and S. Luding, editors, Physics of Dry Granular Media, volume 350 of NATO ASI Series, page 407, Dordrecht, 1998. Kluwer Academic Publishers.

[AGZ98b] T. Aspelmeier, G. Giese, and A. Zippelius. Cooling dynamics of a dilute gas of inelastic rods: a many particle simulation. Phys. Rev. E, 57(1):857, 1998.

[AHZ00] T. Aspelmeier, M. Huthmann, and A. Zippelius. Free cooling of particles with rotational degrees of freedom. In S. Luding and T. Pöschel, editors, Granular Matter, Lecture Notes in Physics, Berlin, to appear in 2000. Springer-Verlag.

[And30] J. D. Andrews. Theory of collision of spheres of soft metal. Phil. Mag. Ser. \%, 9:593, 1930.

[Aue94] D. Auerbach. Colliding rods: Dynamics and relevance to colliding balls. Am. J. Phys., 62:522, 1994.

[AZ00] M. Arndt and A. Zeilinger. Wo ist die Grenze der Quantenwelt? Physikalische Blätter, 56(3), 2000.

[BBW63] S. W. Benson, G. C. Berend, and J. C. Wu. Classical model for vibrational and rotational excitation of diatomic molecules by collision. I. Hard-sphere collision. J. Chem. Phys., 38(1):25, 1963.

[BE98a] R. Brito and M. H. Ernst. Extension of Haff's cooling law in granular flows. Europhys. Lett., 43:497, 1998.

[BE98b] R. Brito and M. H. Ernst. Noise reduction and pattern formation in rapid granular flows. Int. J. Mod. Phys. C, 9(8):1339, 1998.

[Bha99] K. K. Bhattacharya. Simulations of the glassy state of matter. PhD thesis, Universität Göttingen, 1999. 
[BL71] A. Bokor and H. G. Leventhal. The measurement of initial impact velocity and contact time. J. Phys. D, 4:160, 1971.

[BM90] B. Bernu and R. Mazighi. One-dimensional bounce of inelastically colliding marbles on a wall. J. Phys. A, 23(24):5745, 1990.

[Bro96] B. Brogliato. Nonsmooth Impact Mechanics: Models, Dynamics and Control, volume 220 of Lecture Notes in Control and Information Sciences. Springer-Verlag, London, 1996.

[BSHP96] N. V. Brilliantov, F. Spahn, J.-M. Hertzsch, and T. Pöschel. Model for collisions in granular gases. Phys. Rev. E, 53:5382, 1996.

[BSL ${ }^{+96}$ F. G. Bridges, K. D. Supulver, D. N. C. Lin, R. Knight, and M. Zafra. Energy loss and sticking mechanisms in particle aggregation in planetesimal formation. Icarus, 123:422, 1996.

[BWKH96] J. Blum, G. Wurm, S. Kempf, and T. Henning. The Brownian motion of dust particles in the solar nebula: An experimental approach to the problem of pre-planetary dust aggregation. Icarus, 124:441, 1996.

[CC60] S. Chapman and T. G. Cowling. The mathematical theory of nonuniform gases. Cambridge University Press, London, 1960.

[CDKK99] B. Cipra, P. Dini, S. Kennedy, and A. Kolan. Stability of onedimensional inelastic collision sequences of four balls. Physica D, 125(34):183, 1999.

[Cer88] C. Cercignani. The Boltzmann equation and its applications. SpringerVerlag, New York, 1988.

[CLB ${ }^{+}$93] E. Clément, S. Luding, A. Blumen, J. Rajchenbach, and J. Duran. Fluidization, condensation and clusterization of a vibrating column of beads. Int. J. Mod. Phys. B, 7(9 \& 10):1807, 1993.

[CR98] A. Chatterjee and A. Ruina. A new algebraic ridgid-body collision law based on impulse space considerations. J. Appl. Mech., 65:939, 1998.

[CSB98] S. J. Cornell, M. R. Swift, and A. J. Bray. Inelastic collapse of a randomly forced particle. Phys. Rev. Lett., 81(6):1142, 1998.

[DB97] P. Deltour and J.-L. Barrat. Quantitative study of a freely cooling granular medium. J. Phys. I France, 7:137, 1997.

[dG99] P. G. de Gennes. Granular matter: a tentative view. Rev. Mod. Phys., 71(2):S374, 1999. 
[Dil93] J. P. Dilley. Energy loss in collisions of icy spheres: Loss mechanism and size-mass dependence. Icarus, 105:225, 1993.

[EDHvL69] M. H. Ernst, J. R. Dorfman, W. R. Hoegy, and J. M. J. van Leeuwen. Hard-sphere dynamics and binary-collision operators. Physica, 45:127, 1969.

[FFL99] É. Falcon, S. Fauve, and C. Laroche. Cluster formation, pressure and density measurements in a granular medium fluidized by vibrations. Eur. Phys. J. B, 9:183, 1999.

[FLCA94] S. F. Foerster, M. Y. Louge, H. Chang, and K. Allia. Measurements of the collision properties of small spheres. Phys. Fluids, 6(3):1108, 1994.

[FWÉ+99] É. Falcon, R. Wunenburger, P. Évesque, S. Fauve, C. Chabot, Y. Garrabos, and D. Beysens. Cluster formation in a granular medium fluidized by vibrations in low gravity. Phys. Rev. Lett., 83(2):440, 1999.

[Gie96] G. Giese. Dynamik granularer Teilchen mit internen Freiheitsgraden. PhD thesis, Universität Göttingen, 1996.

[Gol60] W. Goldsmith. Impact: The theory and physical behaviour of colliding solids. Edward Arnold Publishers, London, 1960.

[GS95] A. Goldshtein and M. Shapiro. Mechanics of collisional motion of granular materials. Part 1. General hydrodynamic equations. J. Fluid Mech., 282:75, 1995.

[GSB $\left.{ }^{+} 98\right]$ D. Goldman, M. D. Shattuck, C. Bizon, W. D. McCormick, J. B. Swift, and H. L. Swinney. Absence of inelastic collapse in a realistic three ball model. Phys. Rev. E, 57(4):4831, 1998.

[GTZ93] I. Goldhirsch, M.-L. Tan, and G. Zanetti. A molecular dynamical study of granular fluids I: The unforced granular gas in two dimensions. $J$. Sci. Comp., 8(1):1, 1993.

[GZ93] I. Goldhirsch and G. Zanetti. Clustering instability in dissipative gases. Phys. Rev. Lett., 70(11):1619, 1993.

[GZ96] G. Giese and A. Zippelius. Collision properties of one-dimensional granular particles with internal degrees of freedom. Phys. Rev. E, 54(5):4828, 1996.

[GZ99] F. Gerl and A. Zippelius. Coefficient of restitution for elastic disks. Phys. Rev. E, 59(2):2361, 1999. 
[Haf83] P. K. Haff. Grain flow as a fluid-mechanical phenomenon. J. Fluid Mech., 134:401, 1983.

[HAZ99] M. Huthmann, T. Aspelmeier, and A. Zippelius. Granular cooling of hard needles. Phys. Rev. E, 60(1):654, 1999.

[HBL88] A. P. Hatzes, F. G. Bridges, and D. N. C. Lin. Collisional properties of ice spheres at low impact velocities. Mon. Not. R. astr. Soc., 231:1091, 1988.

[Her82] H. Hertz. Über die Berührung fester Körper. J. Reine Angew. Math., 92:156, 1882.

[HHL98] H. J. Herrmann, J.-P. Hovi, and S. Luding, editors. Physics of Dry Granular Media, volume 350 of NATO ASI Series, Dordrecht, 1998. Kluwer Academic Publishers.

[HM86] J.-P. Hansen and I. R. McDonald. Theory of simple liquids. Academic Press, London, 2nd edition, 1986.

[HSB95] J.-M. Hertzsch, F. Spahn, and N. V. Brilliantov. On low-velocity collisions of viscoelastic particles. J. Phys. II France, 5:1725, 1995.

[Hut99] M. Huthmann. Free cooling of granular particles with rotational degrees of freedom. PhD thesis, Universität Göttingen, 1999.

[HZ97] M. Huthmann and A. Zippelius. Dynamics of inelastically colliding rough spheres: relaxation of translational and rotational energy. Phys. Rev. E, 56:R6275, 1997.

[JN92] H. M. Jaeger and S. R. Nagel. Physics of the granular state. Science, 255:1523, 1992 .

[JNB96] H. M. Jaeger, S. R. Nagel, and R. P. Behringer. Granular solids, liquids, and gases. Rev. Mod. Phys., 68(4):1259, 1996.

[JR85a] J. T. Jenkins and M. W. Richman. Grad's 13-moment system for a dense gas of inelastic spheres. Arch. Rat. Mech. Annal., 87:355, 1985.

[JR85b] J. T. Jenkins and M. W. Richman. Kinetic theory for plane flows of a dense gas of identical, rough, inelastic, circular disks. Phys. Fluids, 28:3485, 1985.

[JS83] J. T. Jenkins and S. B. Savage. A theory for the rapid flow of identical, smooth, nearly elastic particles. J. Fluid Mech., 130:187, 1983. 
[Kad99] L. P. Kadanoff. Built upon sand: Theoretical ideas inspired by granular flows. Rev. Mod. Phys., 71(1):435, 1999.

[KK87] G. Kuwabara and K. Kono. Restitution coefficient in a collision between two spheres. Jap. J. Appl. Phys., 26(8):1230, 1987.

[Kol63] H. Kolsky. Stress waves in solids. Dover Publications, New York, 1963.

[Kol83] M. G. Koller. Elastischer Stoß von Kugeln auf dicke Platten. PhD thesis, ETH Zürich, 1983.

[KP92] P. E. Kloeden and E. Platen. Numerical solution of stochastic differential equations. Springer-Verlag, Berlin, 1992.

[Lam82] H. Lamb. On the vibrations of an elastic sphere. Proc. Lond. Math. Soc., xiii:189, 1882.

$\left[\mathrm{LCB}^{+} 94\right] \quad$ S. Luding, E. Clément, A. Blumen, J. Rajchenbach, and J. Duran. Studies of columns of beads under external vibrations. Phys. Rev. E, 49:1634, 1994.

[LHB94] S. Luding, H. J. Herrmann, and A. Blumen. Simulations of twodimensional arrays of beads under external vibrations: Scaling behavior. Phys. Rev. E, 50(4):3100, 1994.

[LHMZ98] S. Luding, M. Huthmann, S. McNamara, and A. Zippelius. Homogeneous cooling of rough, dissipative particles: Theory and simulations. Phys. Rev. E, 58:3416, 1998.

[LL91a] L. D. Landau and E. M. Lifschitz. Elastizitätstheorie, volume 7 of Lehrbuch der Theoretischen Physik. Akademie Verlag, Berlin, 7th edition, 1991.

[LL91b] L. D. Landau and E. M. Lifschitz. Mechanik, volume 1 of Lehrbuch der Theoretischen Physik. Akademie Verlag, Berlin, 7th edition, 1991.

[LM98] S. Luding and S. McNamara. How to handle the inelastic collapse of a dissipative hard-sphere gas with the TC model. Gran. Mat., 1:113, 1998.

[LSJC83] C. K. K. Lun, S. B. Savage, D. J. Jeffrey, and N. Chepurny. Kinetic theories of granular flow: Inelastic particles in a Couette flow and lightly inelastic particles in a general flow field. J. Fluid Mech., 140:223, 1983.

[Lub91] B. D. Lubachevsky. How to simulate billiards and similar systems. $J$. Comp. Phys., 94:255, 1991. 
[Lud98] S. Luding. Collisions \& contacts between two particles. In H. J. Herrmann, J.-P. Hovi, and S. Luding, editors, Physics of Dry Granular Media, volume 350 of NATO ASI Series, page 285, Dordrecht, 1998. Kluwer Academic Publishers.

[Lun91] C. K. K. Lun. Kinetic theory for granular flow of dense, slightly inelastic, slightly rough spheres. J. Fluid Mech., 233:539, 1991.

[McN93] S. McNamara. Hydrodynamic modes of a uniform granular medium. Phys. Fluids, 5(12):3056, 1993.

[ML98] S. McNamara and S. Luding. Energy nonequipartition in systems of inelastic, rough spheres. Phys. Rev. E, 58(2):2247, 1998.

[Mül99] P. Müller. private communication, 1999.

[MY92] S. McNamara and W. R. Young. Inelastic collapse and clumping in a one-dimensional granular medium. Phys. Fluids, 4(3):496, 1992.

[MY93] S. McNamara and W. R. Young. Kinetics of a one-dimensional granular medium in the quasielastic limit. Phys. Fluids, 5(1):34, 1993.

[MY96] S. McNamara and W. R. Young. Dynamics of a freely evolving, twodimensional granular medium. Phys. Rev. E, 53(5):5089, 1996.

[OBvNE97] J. A. G. Orza, R. Brito, T. P. C. van Noije, and M. H. Ernst. Patterns and long range correlations in idealized granular flows. Int. J. Mod. Phys. C, 8(4):953, 1997.

[OU98] J. S. Olafsen and J. S. Urbach. Clustering, order, and collapse in a driven granular monolayer. Phys. Rev. Lett., 81(20):4369, 1998.

[OU99] J. S. Olafsen and J. S. Urbach. Velocity distributions and density fluctuations in a granular gas. Phys. Rev. E, 60(3):R2468, 1999.

[Pao55] Y.-H. Pao. Extension of the Hertz theory of impact to the viscoelastic case. J. Appl. Phys., 26(9):1083, 1955.

$\left[\mathrm{PLM}^{+} 98\right]$ A. Puglisi, V. Loreto, U. M. B. Marconi, A. Petri, and A. Vulpiani. Clustering and non-gaussian behavior in granular matter. Phys. Rev. Lett., 81(18):3848, 1998.

[PTVF92] W. H. Press, S. A. Teukolsky, W. T. Vetterling, and B. P. Flannery. Numerical Recipes in C. Cambridge University Press, Cambridge, 2nd edition, 1992. 
[Ram20] C. V. Raman. On some applications of Hertz's theory of impact. Phys. Rev., 15:277, 1920.

[Ray06] O. M. Rayleigh. On the production of vibrations by forces of relatively long duration. Phil. Mag. Ser. 6, 11:283, 1906.

[Rou98] S. Roux. Quasi-static contacts. In H. J. Herrmann, J.-P. Hovi, and S. Luding, editors, Physics of Dry Granular Media, volume 350 of NATO ASI Series, page 267, Dordrecht, 1998. Kluwer Academic Publishers.

[SB99] M. R: Swift and A. J. Bray. Survival-time distribution for inelastic collapse. Phys. Rev. E, 59(5):R4721, 1999.

[Sch90] F. Scheck. Mechanik. Springer-Verlag, Berlin, 2nd edition, 1990.

[SG95] N. Sela and I. Goldhirsch. Hydrodynamics of a one-dimensional granular medium. Phys. Fluids, 7(3):507, 1995.

[SHB95] F. Spahn, J.-M. Hertzsch, and N. V. Brilliantov. The role of particle collisions for the dynamics in planetary rings. Chaos, Solitons 86 Fractals, 5(10):1945, 1995.

[SJ81] S. B. Savage and D. J. Jeffrey. The stress tensor in a granular flow at high shear rates. J. Fluid Mech., 110:255, 1981.

[SSK97] F. Spahn, U. Schwarz, and J. Kurths. Clustering of granular assemblies with temperature dependent restitution under Keplerian differential rotation. Phys. Rev. Lett., 78:1596, 1997.

[Tab48] D. Tabor. A simple theory of static and dynamic hardness. Proc. $R$. Soc. London Ser. A, 172:247, 1948.

[vNE99] T. P. C. van Noije and M. H. Ernst. Cahn-Hilliard theory for unstable granular flows. cond/mat, 9907012, 1999.

[vNEB98] T. P. C van Noije, M. H. Ernst, and R. Brito. Ring kinetic theory for an idealized granular gas. Physica A, 251:266, 1998.

[WG97] D. E. Wolf and P. Grassberger, editors. Friction, arching, contact dynamics, Singapore, 1997. World Scientific.

[WHJ95] S. Warr, J. M. Huntley, and G. T. H. Jacques. Fluidization of a twodimensional granular system: Experimental studiy and scaling behavior. Phys. Rev. E, 52(5):5583, 1995.

[Wid58] B. Widom. Relaxation of the string oscillator. J. Chem. Phys, 28(5):918, 1958. 
[Wil96] D. R. M. Williams. Driven granular media and dissipative gases: correlations and liquid-gas phase transitions. Physica A, 233:718, 1996.

[WM96] D. R. M. Williams and F. C. MacKintosh. Driven granular media in one dimension: Correlations and equation of state. Phys. Rev. E, 54(1):54, 1996.

[Zen41] C. Zener. The intrinsic inelasticity of large plates. Phys. Rev., 59:669, 1941. 


\section{Acknowledgement}

My thanks go first of all to Prof. Annette Zippelius who gave me all the freedom and support that one could hope for. Prof. Reiner Kree has enlivened my work by helpful physical discussions. My work would not have been half as enjoyable without my collegues and friends Kamal, Kurt, Martin, Henning, Peter, Julia, and all the others, and especially Gisela, who always helped to keep my bureaucratic hassle at a minimum.

This work has been supported by the DFG through grant No. Zi209/5-1. 


\section{Curriculum Vitae}

Born: January 23, 1969 in Gütersloh, Germany

Parents:

Annikki and Dieter Aspelmeier

Country of citizenship: Germany

\section{Studies and training}

April 2000

Dr. rer. nat. in Theoretical Physics

Georg-August-Universität Göttingen

February 1999

through April 1999

Scientific visit to Prof. R.K.P. Zia and Prof. B. Schmittmann,

Virginia Polytechnic Institute and State University, Blacksburg, VA

February 1996

Diplom in Physics

Georg-August-Universität Göttingen

October 1992

Continuation of studies in Edinburgh

through September 1993

University of Edinburgh

June 1991

Vordiplom in Physics

Georg-August-Universität Göttingen

June 1988
Abitur
Gymnasium Verl 


\section{List of publications}

[1] T. Aspelmeier, F. Gerl, and A. Zippelius. A microscopic model of energy dissipation in granular collisions. In H. J. Herrmann, J.-P. Hovi, and S. Luding, editors, Physics of Dry Granular Media, volume 350 of NATO ASI Series, page 407, Dordrecht, 1998. Kluwer Academic Publishers.

[2] T. Aspelmeier, G. Giese, and A. Zippelius. Cooling dynamics of a dilute gas of inelastic rods: a many particle simulation. Phys. Rev. E, 57(1):857, 1998.

[3] M. Huthmann, T. Aspelmeier, and A. Zippelius. Granular cooling of hard needles. Phys. Rev. E, 60(1):654, 1999.

[4] W. Triampo, T. Aspelmeier, and B. Schmittmann. Universal aspects of vacancymediated disordering dynamics: the effect of external fields. Phys. Rev. E, 61(3):2386, 2000.

[5] T. Aspelmeier and A. Zippelius. Dynamics of a one-dimensional granular gas with a stochastic coefficient of restitution. Physica A, 282(3-4):450, 2000.

[6] T. Aspelmeier, M. Huthmann, and A. Zippelius. Free cooling of particles with rotational degrees of freedom. In S. Luding and T. Pöschel, editors, Granular Matter, Lecture Notes in Physics, Berlin, to appear in 2000. Springer-Verlag. 